\title{
11 Special Functions
}

\subsection{Introduction}

The "special functions" of mathematical physics are simply functions that occur so frequently in the solution of physical problems that they have been studied exhaustively resulting in an unusually complete knowledge of their properties. We made the acquaintance of a number of these functions in the last Chapter. Now what we need to do is learn enough about them that acquaintance waxes into friendship or at least into that level of familiarity needed to feel comfortable when they arise in the solution of boundary value problems. And, to promote that sense of comfort, we shall solve problems drawn from several fields of physics. More often than not, the problems will be classified not by their physical origin but by their spatial symmetry.

We shall commence with a study of spherical harmonics.

\subsection{Spherical Harmonics: Problems Possessing Spherical Symmetry}

\subsubsection{Introduction}

As we learned in Section 10.4, spherical harmonics are eigenfunctions of the angular part of the Laplacian differential operator $\nabla^{2}$ when it is expressed in spherical coordinates. Thus, they arise in descriptions of electromagnetic phenomena and of classical and quantum mechanical wave motion. This also means that they are eigenfunctions of the orbital angular momentum operator in quantum mechanics and so they figure in the description of molecules, atoms and nuclei and even in some models of sub-nuclear or quark matter. All of which is to say, spherical harmonics warrant our attention.

We start by reviewing a few lines from Section 10.4. Substitution of a separated solution $u(r, \theta, \varphi)$ or $\psi(r, \theta, \varphi)=R(r) Y(\theta, \varphi)$ into the Helmholtz equation or Laplace's equation resulted in the following equation for $Y$ :

$$
\frac{1}{\sin \theta} \frac{\partial}{\partial \theta}\left(\sin \theta \frac{\partial Y}{\partial \theta}\right)+\frac{1}{\sin ^{2} \theta} \frac{\partial^{2} Y}{\partial \varphi^{2}}=-\lambda_{1} Y(\theta, \varphi) .
$$

Accompanied by suitable boundary conditions, this is an eigenvalue equation and the eigenfunctions are found by performing a second separation of variables.

Specifically, we set $Y(\theta, \varphi)=\Theta(\theta) \Phi(\varphi)$ and obtain

$$
\frac{d^{2} \Phi}{d \varphi^{2}}=-\lambda_{2} \Phi(\varphi)
$$


and

$$
\frac{1}{\sin \theta} \frac{d}{d \theta}\left(\sin \theta \frac{d \Theta}{d \theta}\right)+\left(\lambda_{1}-\frac{\lambda_{2}}{\sin ^{2} \theta}\right) \Theta=0
$$

where $0 \leq \varphi \leq 2 \pi$ and $0 \leq \theta \leq \pi$.

Since we want single-valued solutions we impose the boundary condition $\Phi(\varphi+$ $2 \pi)=\Phi(\varphi)$. This implies that

$$
\lambda_{2}=m^{2} \text { and } \Phi=\Phi_{m}(\varphi)=\frac{1}{\sqrt{2}} e^{i m \varphi}, \quad m=0, \pm 1, \pm 2, \ldots
$$

where we have included a normalization factor.

We now turn our attention to equation (11.2.3) which can be rendered more familiar by a transformation of the independent variable. In terms of the new variable $x=\cos \theta$, (11.2.3) becomes

$$
\left(1-x^{2}\right) \frac{d^{2} P}{d x^{2}}-2 x \frac{d P}{d x}+\left(\lambda-\frac{m^{2}}{1-x^{2}}\right) P=0
$$

where $P(x)=\Theta\left(\cos ^{-1} x\right)$ and $-1 \leq x \leq 1$. Note that we have suppressed the subscript on $\lambda_{1}$. This is a variant of Legendre's equation called the associated Legendre equation. Like the original Legendre DE, which corresponds to setting $m=0$ in (11.2.5), it has regular singular points at $x= \pm 1$. Therefore, the boundary condition that we must impose on its solutions is that they be bounded, $|P( \pm 1)|<\infty$. We shall now use the analytical tools of Chapter 9 to find the eigenfunctions that result from that imposition.

\subsubsection{Associated Legendre Polynomials}

Our first tentative move will be to expand $P(x)$ in Frobenius series about the regular singular points $x= \pm 1$. Our object is to determine its leading behaviour there.

Substituting $P(x)=\sum_{k=0}^{\infty} c_{k}(x-1)^{s+k}, c_{0} \neq 0$ into (11.2.5) and equating the coefficient of the lowest power of $(x-1)$ to zero, we obtain the indicial equation

$$
4 s(s-1)+4 s-m^{2}=0 \text { with roots } s= \pm \frac{m}{2} .
$$

To remove a source of ambiguity, we shall restrict $m \geq 0$ for the time being.

The root $s=-\frac{m}{2}$ can be ruled out immediately because $P(x)$ has to be bounded at $x=1$. Therefore, we must be able to write $P(x)=(1-x)^{\frac{m}{2}} f(x)$ where $f(x)$ is bounded and non-vanishing at $x=1$.

Next, we substitute $P(x)=\sum_{k=0}^{\infty} c_{k}(x+1)^{s+k}, c_{0} \neq 0$ into (11.2.5) and again determine the indicial equation. The result is the same as before and so $s$ must again be set equal to $\frac{m}{2}$. This means that $P(x)=(1+x)^{\frac{m}{2}} g(x)$ where $g(x)$ is bounded and non-vanishing at $x=-1$. 
Combining these two results, we conclude that $P(x)$ can be expressed in the factored form

$$
P(x)=\left(1-x^{2}\right)^{\frac{m}{2}} u(x)
$$

where $u(x)$ must be bounded and non-vanishing at $x= \pm 1$. A differential equation for $u(x)$ can be found by substituting (11.2.6) into the associated Legendre equation. The result is

$$
\left(1-x^{2}\right) \frac{d^{2} u}{d x^{2}}-2(m+1) x \frac{d u}{d x}+\left(\lambda-m-m^{2}\right) u=0 .
$$

Since $x=0$ is an ordinary point, the solutions of this DE will have the Taylor series representation $u(x)=\sum_{k=0}^{\infty} c_{k} x^{k}$. Substitution into (11.2.7) and equation of coefficients of successive powers of $x$ to zero results in the recurrence relation

$$
c_{k+2}=\frac{k(k-1)+2(m+1) k-\lambda+m(m+1)}{(k+1)(k+2)} c_{k}, \quad k \geq 0 .
$$

This relates the coefficients of all even powers in $u(x)$ back to $c_{0}$ and of all odd powers back to $c_{1}$. Thus, as we learned to expect in Chapter 9, we have obtained two linearly independent solutions.

Applying standard convergence tests (the ratio test for example), one finds that the series diverge at $x= \pm 1$. However, there is a remedy at hand and it is one we have invoked before.

If we choose $\lambda$ so that $c_{k+2}=0$ for some $k$, one of the series will terminate to become a polynomial of degree $k$. Therefore, the requirement that $|u( \pm 1)|<\infty$ implies that

$$
k(k-1)+2(m+1) k-\lambda+m(m+1)=0,
$$

or

$$
\lambda=(m+k)(m+k+1) \text { for some } k .
$$

This has a familiar ring to it and to reinforce the familiarity we set $m+k=l$. The eigenvalues $\lambda$ are then specified as

$$
\lambda=l(l+1), \quad l \geq m, m=0,1,2, \ldots .
$$

The corresponding solutions for $u(x)$ are polynomials of degree $l-m$.

Normally, the next step would be to use the recurrence relation (11.2.8) to determine an explicit expression for $u(x)$. However, it is less messy as well as more instructive to make the determination in a somewhat different way. As we know from Chapter 9, Legendre polynomials satisfy the equation

$$
\left(1-x^{2}\right) \frac{d^{2} P_{l}}{d x^{2}}-2 x \frac{d P_{l}}{d x}+l(l+1) P_{l}=0 .
$$


Differentiating $m$ times we obtain

$$
\left(1-x^{2}\right) \frac{d^{2}}{d x^{2}}\left(\frac{d^{m} P_{l}}{d x^{m}}\right)-2(m+1) x \frac{d}{d x}\left(\frac{d^{m} P_{l}}{d x^{m}}\right)+\left[l(l+1)-m-m^{2}\right]\left(\frac{d^{m} P_{l}}{d x^{m}}\right)=0
$$

which is identical to the DE for $u(x)$. This enables us to make the identification $u(x)=$ $\frac{d^{m}}{d x^{m}} P_{l}(x)$ and conclude that the eigenfunction solutions of the associated Legendre equation are

$$
P_{l}^{m}(x)=\left(1-x^{2}\right)^{\frac{m}{2}} \frac{d^{m}}{d x^{m}} P_{l}(x), \quad l=0,1,2, \ldots \text { and } 0 \leq m \leq l
$$

corresponding to the eigenvalues $\lambda=l(l+1)$. These functions are called associated Legendre polynomials (but are polynomials only when $m$ is even). We shall begin an exploration of their properties by focussing on the subset we have met before, the $(m=0)$ Legendre polynomials.

\subsubsection{Properties of Legendre Polynomials}

The explicit polynomial expression for $P_{l}(x)$ that we found in Section 9.6 reads

$$
P_{l}(x)=\frac{1}{2^{l}} \sum_{k=0}^{[l / 2]}(-1)^{k} \frac{(2 l-2 k) !}{k !(l-2 k) !(l-k) !} x^{l-2 k}
$$

where $[l / 2]=\frac{l}{2}$ if $l$ is even and $[l / 2]=\frac{l-1}{2}$ if $l$ is odd . This can be recast in a form that has a variety of uses, both practical and theoretical, by noticing first that

$$
P_{l}(x)=\frac{1}{2^{l}} \frac{d^{l}}{d x^{l}}\left[\sum_{k=0}^{[l / 2]}(-1)^{k} \frac{1}{k !(l-k) !} x^{2 l-2 k}\right]
$$

and then that

$$
\left(x^{2}-1\right)^{l}=\sum_{k=0}^{[l / 2]}(-1)^{k} \frac{l !}{k !(l-k) !} x^{2 l-2 k} .
$$

Combined, these two identities give us Rodrigues' formula for $P_{l}(x)$,

$$
P_{l}(x)=\frac{1}{2^{l} l !} \frac{d^{l}}{d x^{l}}\left(x^{2}-1\right)^{l} .
$$

This formula was derived by Olinde Rodrigues (1795-1851) and appears in his doctoral thesis. After graduation from Université de Paris in 1815, Rodrigues became a banker, a not uncommon fate for mathematicians then as now.

One can generate the lowest order polynomials fairly easily from Rodrigues' formula and thus confirm what we found in Section 7.6:

$$
P_{0}(x)=1, \quad P_{1}(x)=x, \quad P_{2}(x)=\frac{1}{2}\left(3 x^{2}-1\right), \quad P_{2}(x)=\frac{1}{2}\left(5 x^{3}-3 x\right), \ldots
$$


Yet another way of generating the polynomials and more importantly, of deducing many of their properties is to make use of the so-called generating function

$$
G(x, t) \equiv \frac{1}{\sqrt{1-2 x t+t^{2}}}=\sum_{l=0}^{\infty} t^{l} P_{l}(x), \quad|t|<1,|x| \leq 1 .
$$

The proof of this identity can be established by a "brute-force" method that begins with the expansion

$$
\left(1-2 x t+t^{2}\right)^{-\frac{1}{2}}=[1+t(t-2 x)]^{-\frac{1}{2}}=\sum_{m=0}^{\infty} \frac{\left(-\frac{1}{2}\right)\left(-\frac{3}{2}\right) \ldots\left(\frac{1}{2}-m\right)}{m !} t^{m}(t-2 x)^{m},
$$

uses the binomial theorem to expand $(t-2 x)^{m}$, and then performs an "inspired" change of summation index to obtain

$$
G(x, t)=\sum_{l=0}^{\infty} \sum_{k=0}^{[l / 2]}\left(-\frac{1}{2}\right)^{l-k} \frac{1 \cdot 3 \cdot \ldots(2 l-2 k-3)(2 l-2 k-1)}{k !(l-2 k) !} t^{l}(-2 x)^{l-2 k}
$$

which is recognizable, after some simplifiation, as the left hand side of (11.2.14). We shall establish it by a more elegant approach that employs integral representations of $P_{l}(x)$.

A contour integral representation follows immediately from Rodrigues' formula and the Cauchy differentiation formula:

$$
P_{l}(z)=\frac{1}{2^{l}} \frac{1}{2 \pi i} \int_{C} \frac{\left(\zeta^{2}-1\right)^{l}}{(\zeta-z)^{l+1}} d \zeta
$$

where $C$ is any simple closed contour enclosing the point $\zeta=z$. This is called Schläfli's integral representation.

Ludwig Schläfli (1814-1895) was a Swiss geometer and complex analyst.

We shall choose $C$ to be a circle about $z$ with radius $\left|\sqrt{z^{2}-1}\right|$ in which case any point on $C$ is defined by

$$
\zeta=z+\sqrt{z^{2}-1} e^{i \theta}, \quad 0 \leq \theta \leq 2 \pi .
$$

It does not matter which branch of $\sqrt{z^{2}-1}$ is used here so long as we are consistent. A little algebraic manipulation then gives us

$$
\zeta^{2}-1=2(\zeta-z)\left(z+\sqrt{z^{2}-1} \cos \theta\right) \text { and } d \zeta=i(\zeta-z) d \theta
$$

Therefore, substituting into (11.2.15), we obtain Laplace's integral representation,

$$
P_{l}(z)=\frac{1}{\pi} \int_{0}^{\pi}\left(z+\sqrt{z^{2}-1} \cos \theta\right)^{l} d \theta
$$


This is the representation we need for the generating function identity. Substituting (11.2.16) into the right hand side of (11.2.14) and interchanging the order of summation and integration, we have

$$
\begin{aligned}
\sum_{l=0}^{\infty} t^{l} P_{l}(x) & =\frac{1}{\pi} \int_{0}^{\pi} \sum_{l=0}^{\infty} t^{l}\left(x+\sqrt{x^{2}-1} \cos \theta\right)^{l} d \theta \\
& =\frac{1}{\pi} \int_{0}^{\pi} \frac{d \theta}{1-t x-t \sqrt{x^{2}-1} \cos \theta} \\
& =\frac{1}{\sqrt{1-2 t x+t^{2}}}=G(x, t),
\end{aligned}
$$

where the evaluation of the integral over $\theta$ is done by residue calculus (or by reference to a set of integral tables). This is just (11.2.14) written in reverse order and so our derivation is complete.

The generating function readily yields the values assumed by $P_{l}(x)$ at a number of special points. For example, at $x=1$, we have

$$
\sum_{l=0}^{\infty} t^{l} P_{l}(1)=G(1, t)=\frac{1}{1-t}=\sum_{l=0}^{\infty} t^{l}, \quad|t|<1 .
$$

Therefore,

$$
P_{l}(1)=1 \text { for all } l \geq 0 \text {. }
$$

At $x=0$, we have

$$
\sum_{l=0}^{\infty} t^{l} P_{l}(0)=\left(1+t^{2}\right)^{-\frac{1}{2}}=1-\frac{1}{2} t^{2}+\left(-\frac{1}{2}\right)\left(-\frac{3}{2}\right) \frac{t^{4}}{2}+\ldots
$$

Therefore,

$$
P_{l}(0)=\left\{\begin{array}{cl}
0 & \text { if } l \text { is odd } \\
\frac{(-1)^{\frac{l}{2}} l !}{2^{l}\left(\frac{l}{2}\right)^{2}} & \text { if } l \text { is even }
\end{array} .\right.
$$

At $x=-1$, we have

$$
\sum_{l=0}^{\infty} t^{l} P_{l}(-1)=\frac{1}{1+t}=\sum_{l=0}^{\infty}(-t)^{l}
$$

and so,

$$
P_{l}(-1)=(-1)^{l} \text { for all } l \geq 0 \text {. }
$$


More generally,

$$
P_{l}(-x)=(-1)^{l} P_{l}(x)
$$

which follows from

$$
G(-x,-t)=\frac{1}{\sqrt{1-2(-x)(-t)+(-t)^{2}}}=G(x, t)
$$

or

$$
\sum_{l=0}^{\infty} P_{l}(-x)(-t)^{l}=\sum_{l=0}^{\infty} P_{l}(x) t^{l}
$$

The generating function is also the source of a number of useful identities connecting Legendre polynomials of different orders. These are found by differentiating $G(x, t)$.

Differentiating with respect to $t$, we obtain

$$
\frac{\partial G}{\partial t}=\frac{x-t}{\left(1-2 x t+t^{2}\right)^{\frac{3}{2}}}=\frac{x-t}{\left(1-2 t x+t^{2}\right)} G(x, t)=\sum_{l=0}^{\infty} l t^{l-1} P_{l}(x) .
$$

After cross-multiplying and then substituting for $G(x, t)$, this yields

$$
(x-t) \sum_{l=0}^{\infty} t^{l} P_{l}(x)=\left(1-2 x t+t^{2}\right) \sum_{l=0}^{\infty} l t^{l-1} P_{l}(x)
$$

or

$$
\sum_{l=0}^{\infty} l t^{l-1} P_{l}(x)-\sum_{l=0}^{\infty}(2 l+1) x t^{l} P_{l}(x)+\sum_{l=0}^{\infty}(l+1) t^{l+1} P_{l}(x)=0 .
$$

Equating coefficients of like powers of $t$, we find that this implies

$$
(2 l+1) x P_{l}(x)=(l+1) P_{l+1}(x)+l P_{l-1}(x), \quad l=1,2,3, \ldots .
$$

This is called a recursion relation. An immediate application of it is to determine all Legendre polynomials from a knowledge of $P_{0}(x)=1$ and $P_{1}(x)=x$.

If we differentiate $G(x, t)$ with respect to $x$ rather than $t$, we obtain

$$
\frac{\partial G}{\partial x}=\frac{t}{\left(1-2 x t+t^{2}\right)^{\frac{3}{2}}}=\frac{t}{1-2 x t+t^{2}} G(x, t)=\sum_{l=0}^{\infty} t^{l} P_{l}^{\prime}(x), \quad P_{l}^{\prime}(x) \equiv \frac{d P_{l}}{d x} .
$$

Cross-multiplication followed by substitution for $G(x, t)$ makes this read

$$
\sum_{l=0}^{\infty} t^{l+1} P_{l}(x)=\left(1-2 x t+t^{2}\right) \sum_{l=0}^{\infty} t^{l} P_{l}^{\prime}(x) .
$$

Equating coefficients of like powers of $t$, we find the second recursion relation:

$$
P_{l}(x)=P_{l+1}^{\prime}(x)-2 x P_{l}^{\prime}(x)+P_{l-1}^{\prime}(x), \quad l=1,2,3, \ldots
$$


Many other recursion relations can be derived from linear combinations of these two basic ones including, for example,

$$
\begin{aligned}
(2 l+1) P_{l}(x) & =P_{l+1}^{\prime}(x)-P_{l-1}^{\prime}(x) \\
l P_{l}(x) & =x P_{l}^{\prime}(x)-P_{l-1}^{\prime}(x) \\
P_{l+1}^{\prime}(x) & =x P_{l}^{\prime}(x)+(l+1) P_{l}(x) \\
\left(x^{2}-1\right) P_{l}^{\prime}(x) & =l x P_{l}(x)-l P_{l-1}(x) .
\end{aligned}
$$

We now turn our attention to those properties of Legendre polynomials which bear directly on their relevance in the solution of boundary value problems. These are orthogonality, normalization and completeness. Since the polynomials are solutions of the Sturm-Liouville eigenvalue problem

$$
\frac{d}{d x}\left[\left(1-x^{2}\right) \frac{d P_{l}}{d x}\right]=-l(l+1) P_{l}(x) \text { with }\left|P_{l}( \pm 1)\right|<\infty,
$$

they are mutually orthogonal with respect to the weight function $\rho(x)=1$ :

$$
\int_{-1}^{1} P_{l}(x) P_{m}(x) d x=0 \text { if } l \neq m .
$$

Verifying this provides an instructive example of the utility of Rodrigue's formula. Taking $l<m$, we have

$$
\int_{-1}^{1} P_{l}(x) P_{m}(x) d x=\frac{1}{2^{l+m}} \frac{1}{l ! m !} \int_{-1}^{1}\left[\frac{d^{l}}{d x^{l}}\left(x^{2}-1\right)^{l}\right]\left[\frac{d^{m}}{d x^{m}}\left(x^{2}-1\right)^{m}\right] d x .
$$

Integrating by parts, we obtain

$$
\begin{aligned}
\int_{-1}^{1} \frac{d^{l}}{d x^{l}}\left(x^{2}-1\right)^{l} & \frac{d^{m}}{d x^{m}}\left(x^{2}-1\right)^{m} d x \\
& =\left.\frac{d^{l-1}}{d x^{l-1}}\left(x^{2}-1\right)^{l} \frac{d^{m}}{d x^{m}}\left(x^{2}-1\right)^{m}\right|_{x=-1} ^{x=1} \\
& -\int_{-1}^{1} \frac{d^{l}}{d x^{l}}\left(x^{2}-1\right)^{l} \frac{d^{m+1}}{d x^{m+1}}\left(x^{2}-1\right)^{m} d x .
\end{aligned}
$$

The integrated term vanishes because $\frac{d^{l-1}}{d x^{l-1}}\left(x^{2}-1\right)^{l}$ has simple zeros at the end-points $x= \pm 1$. We now repeat the integration by parts $l-1$ times. In each of these integrations the integrated term vanishes and we are left with

$$
\int_{-1}^{1} \frac{d^{l}}{d x^{l}}\left(x^{2}-1\right)^{l} \frac{d^{m}}{d x^{m}}\left(x^{2}-1\right)^{m} d x=(-1)^{l} \int_{-1}^{1}\left(x^{2}-1\right) \frac{d^{l+m}}{d x^{l+m}}\left(x^{2}-1\right)^{m} d x .
$$


The function $\left(x^{2}-1\right)^{m}$ is a polynomial of degree $2 m$. Thus, if $l>m$, we are differentiating it more than $2 m$ times and so the result is zero. Therefore,

$$
\int_{-1}^{1} P_{l}(x) P_{m}(x) d x=0, \quad l \neq m .
$$

If $l$ does equal $m$, this integral becomes the normalization integral

$$
N_{l}=\int_{-1}^{1}\left[P_{l}(x)\right]^{2} d x=\frac{1}{2^{2 l}} \frac{1}{(l !)^{2}}(-1)^{l} \int_{-1}^{1}\left(x^{2}-1\right)^{l} \frac{d^{2 l}}{d x^{2 l}}\left(x^{2}-1\right)^{l} d x
$$

where we have used (11.2.26) and (11.2.27) with $m=l$. We know that

$$
\frac{d^{2 l}}{d x^{2 l}}\left(x^{2}-1\right)^{l}=(2 l) !
$$

and so,

$$
N_{l}=(-1)^{l} \frac{(2 l) !}{2^{2 l}(l !)^{2}} \int_{-1}^{1}\left(x^{2}-1\right)^{l} d x
$$

The latter integral can be evaluated by repeated integration by parts. One finds

$$
\int_{-1}^{1}\left(x^{2}-1\right)^{l} d x=(-1)^{l} \frac{2^{l+1} l !}{1 \cdot 3 \cdot 5 \cdots(2 l+1)}
$$

Thus,

$$
\int_{-1}^{1}\left[P_{l}(x)\right]^{2} d x=\frac{2}{2 l+1} .
$$

The normalization integral can also be derived from the generating function. Specifically, we can square $G(x, t)$,

$$
[G(x, t)]^{2}=\frac{1}{1-2 x t+t^{2}}=\left[\sum_{l=0}^{\infty} t^{l} P_{l}(x)\right]^{2}=\sum_{l=0}^{\infty} \sum_{m=0}^{\infty} t^{l+m} P_{l}(x) P_{m}(x),
$$

and then integrate from -1 to 1 to obtain

$$
\int_{-1}^{1} \frac{d x}{1-2 x t+t^{2}}=\sum_{l=0}^{\infty} \sum_{m=0}^{\infty} t^{l+m} \int_{-1}^{1} P_{l}(x) P_{m}(x) d x=\sum_{l=0}^{\infty} t^{2 l} \int_{-1}^{1}\left[P_{l}(x)\right]^{2} d x
$$

where we have used the orthogonality of the Legendre polynomials to eliminate all but the terms with $m=l$ on the right hand side of the equation. Introducing a new variable of integration $y=1-2 t x+t^{2}$ the left hand side of the equation is found to be

$$
\int_{-1}^{1} \frac{d x}{1-2 t x+t^{2}}=\frac{1}{2 t} \int_{(1-t)^{2}}^{(1+t)^{2}} \frac{d y}{y}=\frac{1}{t} \ln \left(\frac{1+t}{1-t}\right)
$$


which has the power series expansion

$$
\frac{1}{t} \ln \left(\frac{1+t}{1-t}\right)=2 \sum_{l=0}^{\infty} \frac{t^{2 l}}{2 l+1}
$$

Equating coefficients of like powers of $t$ in (11.2.29) and (11.2.30), we recover

$$
N_{l}=\int_{-1}^{1}\left[P_{l}(x)\right]^{2} d x=\frac{2}{2 l+1}
$$

Orthogonality and normalization are conveniently combined in the single equation

$$
\int_{-1}^{1} P_{l}(x) P_{m}(x) d x=\frac{2}{2 l+1} \delta_{l, m} .
$$

As eigenfunctions of a Sturm-Liouville problem, Legendre polynomials form a complete set: any function $f(x)$ that is square-integrable with respect to the weight $\rho(x)=1$ on the interval $-1 \leq x \leq 1$ can be represented on that interval by the expansion

$$
f(x)=\sum_{l=0}^{\infty} c_{l} P_{l}(x) \text { where } c_{l}=\frac{2 l+1}{2} \int_{-1}^{1} P_{l}\left(x^{\prime}\right) f\left(x^{\prime}\right) d x^{\prime} .
$$

This is sometimes called a Fourier-Legendre series. The completeness relation for Legendre polynomials is

$$
\sum_{l=0}^{\infty} \frac{2}{2 l+1} c_{l}^{2}=\int_{-1}^{1}[f(x)]^{2} d x
$$

and the closure relation is

$$
\sum_{l=0}^{\infty} \frac{2 l+1}{2} P_{l}(x) P_{l}\left(x^{\prime}\right)=\delta\left(x-x^{\prime}\right) .
$$

In theory at least, the coefficients of like powers in an expansion over an infinite set of polynomials can always be summed so that the expansion is converted to a power series. This raises the question of how power series fit within what is in fact an algebraic picture. The answer is straightforward but instructive. A Taylor series about $x=0$ is an expansion over the monomials $1, x, x^{2}, x^{3}, \ldots$ and the algebraic counterpart of Taylor's Theorem is a theorem due to Weierstrass that this set is complete with respect to any space of square integrable functions. However, they do not form an orthogonal set. Rather, once a weight function and interval of definition is given, one has to form orthogonal linear combinations of the monomials. There are well-defined 
methods for doing so, the most general being the Gram-Schmidt orthogonalization procedure which constructs successively polynomials of degree $l$ that are orthogonal to the polynomials of degree $0,1,2, \ldots l-1$. The outcome is a set of orthogonal polynomials that is unique to the choice of interval and weight function used. We will discuss this in more detail in a subsequent section. For now it suffices to know that the orthogonalization process in the case of the interval $-1 \leq x \leq 1$ and weight $\rho(x)=1$ results in the Legendre polynomials.

\subsubsection{Problems Possessing Azimuthal Symmetry}

Returning to the discussion in Section 11.2.1 of what happens with separation of variables in problems with spherical symmetry, we now know that the angular dependence will be governed by the complete set of functions

$$
P_{l}^{m}(\cos \theta)\left\{\begin{array}{c}
\cos m \varphi \\
\sin m \varphi
\end{array}\right\} l=0,1,2, \ldots \text { and } 0 \leq m \leq l
$$

where we have used an explicitly real form for $\Phi_{m}(\varphi)$.

Many applications involve symmetry about the $\mathrm{z}$-axis. This means that there will be no dependence on the azimuthal angle $\varphi$ and so it is often referred to as azimuthal symmetry. A glance at (11.2.35) tells us that no $\varphi$-dependence necessarily implies $m=$ 0 and hence, a $\theta$ - dependence expressible in terms of Legendre polynomials rather than associated Legendre polynomials. In the case of Laplace's equation, for example, a solution with azimuthal symmetry will have the Fourier-Legendre expansion

$$
\psi(r, \theta)=\sum_{l=0}^{\infty} R_{l}(r) P_{l}(\cos \theta)
$$

where, as we saw in Section 10.4, $R_{l}(r)$ is a solution of

$$
\frac{d^{2} R_{l}}{d r^{2}}+\frac{2}{r} \frac{d R_{l}}{d r}-\frac{l(l+1)}{r^{2}} R_{l}=0 .
$$

The general solution of this equation is

$$
R_{l}(r)=A_{l} r^{l}+B_{l} r^{-l-1}
$$

As a concrete example, suppose that we have two conducting hemispherical shells of radius $b$, insulated from each other by a thin strip along their circle of contact and maintained at potentials $+V$ and $-V$, respectively. We seek the potential everywhere inside the composite sphere.

This is an example of an interior problem: the lower limit of the range for $r$ is zero and, since we require a solution that is bounded there, we can set $B_{l}=0$ for all $l$. (The corresponding exterior problem would be to find the potential everywhere outside 


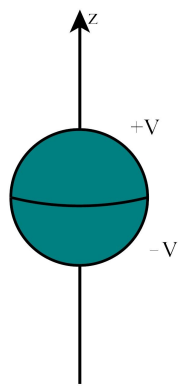

the sphere. In that case, the requirement of a solution that is zero at infinity results in $A_{l}=0$ for all $l$ and the $B_{l}$ have to be determined from the potential at the surface of the sphere.) Thus, it only remains to impose the boundary condition

$$
\psi(b, \theta)= \begin{cases}+V, & 0 \leq \theta<\frac{\pi}{2} \\ -V, & \frac{\pi}{2}<\theta \leq \pi\end{cases}
$$

on the Fourier-Legendre series

$$
\psi(r, \theta)=\sum_{l=0}^{\infty} A_{l} r^{l} P_{l}(\cos \theta) .
$$

Using (11.2.32), we find that this implies

$$
A_{l}=\frac{1}{b^{l}} \frac{2 l+1}{2} \int_{0}^{\pi} \psi(b, \theta) P_{l}(\cos \theta) \sin \theta d \theta
$$

and, since $\psi(b, \theta)$ is an odd function of $\cos \theta$ while $P_{l}(\cos \theta)$ has parity $(-1)^{l}$, this becomes

$$
A_{l}=\left\{\begin{array}{cl}
\frac{1}{b^{l}} \frac{2 l+1}{2} 2 V \int_{0}^{1} P_{l}(\cos \theta) d(\cos \theta) & \text { if } l \text { is odd } \\
0 & \text { if } l \text { is even }
\end{array}\right.
$$

Recalling (from (11.2.23)) that

$$
(2 l+1) P_{l}(x)=P_{l+1}^{\prime}(x)-P_{l-1}^{\prime}(x),
$$

we find

$$
\int_{0}^{1} P_{l}(\cos \theta) d(\cos \theta)=\frac{1}{2 l+1}\left[P_{l+1}(x)-P_{l-1}(x)\right]_{x=0}^{x=1} .
$$

But, $P_{l}(1)=1$ for all $l$ and $P_{l}(0)=\frac{(-1)^{\frac{l}{2}} l !}{2^{l}\left(\frac{l}{2}\right)^{2}}$ if $l$ is even and is zero otherwise. Thus,

$$
\int_{0}^{1} P_{l}(\cos \theta) d(\cos \theta)=\frac{(-1)^{\frac{l+1}{2}}(l+1) !}{2^{l+1}\left(\frac{l+1}{2}\right)^{2} l} \quad(l \text { odd }) .
$$


Therefore, the electrostatic potential at any point inside the sphere is

$$
\psi(r, \theta)=V \sum_{l=1,3,5, \ldots}^{\infty} \frac{(2 l+1)(-1)^{\frac{l-1}{2}}(l+1) !}{2^{l+1}\left(\frac{l+1}{2}\right)^{2} l}\left(\frac{r}{b}\right)^{l} P_{l}(\cos \theta) .
$$

As a further example we shall consider the problem of a conducting sphere in a uniform electric field $\boldsymbol{E}_{0}$. What we seek is the new, perturbed electrostatic potential $\psi$.

Because there are no charges present, $\nabla^{2} \psi=0$. And, choosing our z-axis to be in the direction of $\boldsymbol{E}_{0}$, we have azimuthal symmetry. Thus,

$$
\psi(r, \theta)=\sum_{l=0}^{\infty}\left[A_{l} r^{l}+B_{l} r^{-l-1}\right] P_{l}(\cos \theta)
$$

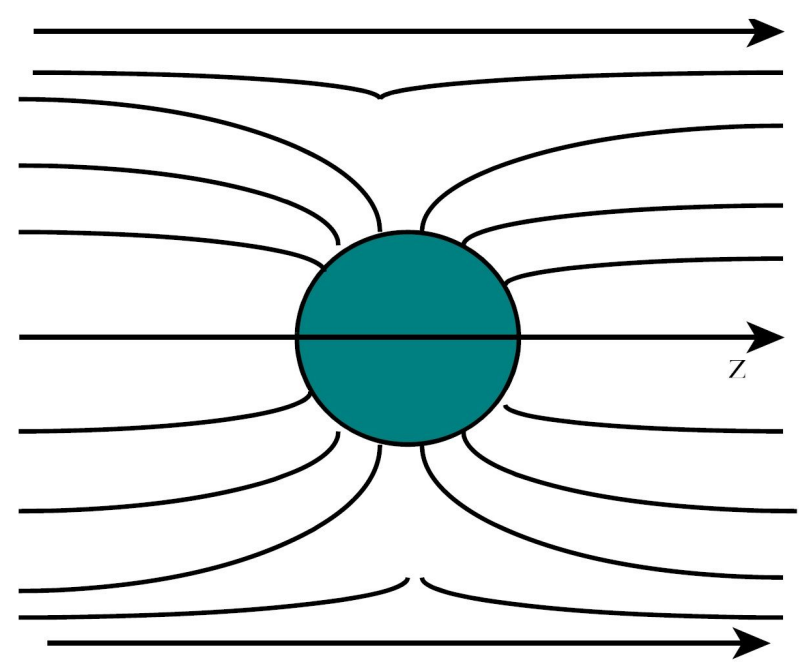

Taking the origin to be at the centre of the sphere, the effect of the perturbing sphere should go to zero as $r \rightarrow \infty$. Therefore, we require

$$
\lim _{r \rightarrow \infty} \psi(r, \theta)=-E_{0} z=-E_{0} r \cos \theta=-E_{0} r P_{1}(\cos \theta) .
$$

It then follows that $A_{l}=0$ for all $l \geq 2$ (as one would expect for an exterior problem) and $A_{1}=-E_{0}$.

The conducting sphere must be at a constant value of potential and so, denoting the sphere's radius by $a$, we have the boundary condition

$$
\psi(a, \theta)=a \text { constant }=A_{0}+\frac{B_{0}}{a}+\left(\frac{B_{1}}{a^{2}}\right) P_{1}(\cos \theta)+\sum_{l=2}^{\infty} B_{l} \frac{P_{l}(\cos \theta)}{a^{l+1}} .
$$


In order that this hold for all $\theta$, each coefficient of a $P_{l}(\cos \theta)$ with $l \neq 0$ must vanish. Thus, $B_{l}=0$ for $l \geq 2$ and $B_{1}=E_{0} a^{3}$ which gives us

$$
\psi(r, \theta)=A_{0}+\frac{B_{0}}{r}-E_{0} r\left(1-\frac{a^{3}}{r^{3}}\right) P_{1}(\cos \theta) .
$$

Since it is $\boldsymbol{E}=\boldsymbol{\nabla} \psi$ rather than $\psi$ itself that has physical significance, the constant $A_{0}$ can be dropped. In addition, we know from Gauss' Law that $B_{0}$ is determined by the net charge $Q$ on the sphere: $B_{0}=\frac{Q}{4 \pi \varepsilon_{0}}$. Therefore, our final answer is

$$
\psi(r, \theta)=\frac{1}{4 \pi \varepsilon_{0}} \frac{Q}{r}-E_{0} r\left(1-\frac{a^{3}}{r^{3}}\right) \cos \theta .
$$

In effect, the sphere has perturbed the external field by adding both a monopole term, $\frac{1}{4 \pi \varepsilon_{0}} \frac{Q}{r}$, and a dipole term, $E_{0} \frac{a^{3}}{r^{2}} \cos \theta$, corresponding to an induced dipole moment

$$
p=a^{3} E_{0} .
$$

\subsubsection{Properties of the Associated Legendre Polynomials}

From (11.2.11) and the Rodrigues' formula for $P_{l}(x)$, we can write down the corresponding formula for $P_{l}^{m}(x)$ immediately:

$$
P_{l}^{m}(x)=\frac{\left(1-x^{2}\right)^{\frac{m}{2}}}{2^{l} l !} \frac{d^{l+m}}{d x^{l+m}}\left(x^{2}-1\right)^{l}, \quad m=0,1,2, \ldots, l, \quad l=0,1,2, \ldots .
$$

This identity yields well-defined functions even if $\mathbf{m}$ is negative, provided that $|m| \leq$ $l$. However, they are not independent of their positive $m$ counterparts since one can show that

$$
P_{l}^{-m}(x)=(-1)^{m} \frac{(l-m) !}{(l+m) !} P_{l}^{m}(x), \quad 0 \leq m \leq l .
$$

From the recursion formulas for $P_{l}(x)$ one can readily obtain formulas for $P_{l}^{m}(x)$. Of particular use are the $\mathbf{m}$-raising and $\mathbf{m}$-lowering relations,

$$
\begin{aligned}
& m x P_{l}^{m}(x)+\left(1-x^{2}\right) \frac{d P_{l}^{m}}{d x}=\left(1-x^{2}\right)^{\frac{1}{2}} P_{l}^{m+1}(x) \\
& m x P_{l}^{m}(x)-\left(1-x^{2}\right) \frac{d P_{l}^{m}}{d x}=(l+m)(l-m+1)\left(1-x^{2}\right)^{\frac{1}{2}} P_{l}^{m-1}(x),
\end{aligned}
$$

and the l-raising and l-lowering relations,

$$
\begin{gathered}
(l+1) x P_{l}^{m}(x)-\left(1-x^{2}\right) \frac{d P_{l}^{m}}{d x}=(l-m+1) P_{l+1}^{m}(x) \\
l x P_{l}^{m}(x)+\left(1-x^{2}\right) \frac{d P_{l}^{m}}{d x}=(l+m) P_{l-1}^{m}(x) .
\end{gathered}
$$


As for parity, we know already that $P_{l}(-x)=(-1)^{l} P_{l}(x)$. Therefore, since

$$
\begin{gathered}
\frac{d^{m}}{d(-\chi)^{m}}=(-1)^{m} \frac{d^{m}}{d x^{m}}, \\
P_{l}^{m}(-\chi)=(-1)^{l+m} P_{l}^{m}(x) .
\end{gathered}
$$

And, in regard to special values, it is obvious that $P_{l}^{m}( \pm 1)=0$.

Of most interest to us are the properties the associated Legendre polynomials have by dint of being eigenfunction solutions of a Sturm-Liouville problem. As we have seen, the equation

$$
\frac{d}{d x}\left[\left(1-x^{2}\right) \frac{d P}{d x}\right]+\left[\lambda-\frac{m^{2}}{1-x^{2}}\right] P=0
$$

together with the boundary condition $|P( \pm 1)|<\infty$ has the eigensolutions $P(x)=$ $P_{l}^{m}(x), 0 \leq m \leq l$, corresponding to eigenvalues $\lambda=l(l+1), l=0,1,2, \ldots$ Thus, the set $P_{l}^{m}(x), l=m, m+1, m+2, \ldots$ for fixed $m$ is complete and orthogonal with respect to the weight function $\rho(x)=1$ on the interval $-1 \leq x \leq 1$.

The statement of orthogonality is

$$
\int_{-1}^{1} P_{l}^{m}(x) P_{k}^{m}(x) d x=0 \text { for all } l \neq k .
$$

The associated Legendre polynomials satisfy a second orthogonality relation. It arises because

$$
\frac{d}{d x}\left[\left(1-x^{2}\right) \frac{d^{2} P_{l}^{m}}{d x}\right]+l(l+1) P_{l}^{m}(x)=\frac{m^{2}}{1-x^{2}} P_{l}^{m}(x), \quad\left|P_{l}^{m}( \pm 1)\right|<\infty
$$

is also an eigenvalue problem with eigenvalues, for fixed $l, \lambda=-m^{2}, 0 \leq m \leq l$, and weight function $\rho(x)=\frac{1}{1-x^{2}}$. Thus,

$$
\int_{-1}^{1} \frac{P_{l}^{m}(x) P_{l}^{n}(x)}{1-x^{2}} d x=0 \text { for all } m \neq n .
$$

Orthogonality needs to be accompanied by knowledge of the corresponding normalization integral. For the physically relevant case, this means that we need to evaluate

$$
N_{l, m}=\int_{-1}^{1}\left[P_{l}^{m}(x)\right]^{2} d x=\int_{-1}^{1}\left(1-x^{2}\right)^{m} \frac{d^{m} P_{l}}{d x^{m}} \frac{d^{m} P_{l}}{d x^{m}} d x .
$$

Integrating by parts, we have

$$
N_{l, m}=\left.\left(1-x^{2}\right)^{m} \frac{d^{m} P_{l}}{d x^{m}} \frac{d^{m-1} P_{l}}{d x^{m-1}}\right|_{-1} ^{1}-\int_{-1}^{1} \frac{d}{d x}\left[\left(1-x^{2}\right)^{m} \frac{d^{m} P_{l}}{d x^{m}}\right] \frac{d^{m-1} P_{l}}{d x^{m-1}} d x
$$


The integrated term is zero and the derivative in the integral is

$$
\frac{d}{d x}\left[\left(1-x^{2}\right)^{m} \frac{d^{m} P_{l}}{d x^{m}}\right]=-2 m x\left(1-x^{2}\right)^{m-1} \frac{d^{m} P_{l}}{d x^{m}}+\left(1-x^{2}\right)^{m} \frac{d^{m+1} P_{l}}{d x^{m+1}}
$$

which we can simplify by using Legendre's equation. Differentiating (11.2.10) $m-1$ times we have

$$
\left(1-x^{2}\right) \frac{d^{m+1} P_{l}}{d x^{m+1}}-2 m x \frac{d^{m} P_{l}}{d x^{m}}=-[l(l+1)-m(m-1)] \frac{d^{m-1} P_{l}}{d x^{m-1}} .
$$

Thus,

$$
N_{l, m}=[l(l+1)-m(m-1)] \int_{-1}^{1}\left(1-x^{2}\right)^{m-1}\left[\frac{d^{m-1} P_{l}}{d x^{m-1}}\right]^{2} d x
$$

The numerical factor in front of the integral can be rearranged to read $(l+m)(l-m+1)$ while the integral itself is recognizable as

$$
N_{l, m-1}=\int_{-1}^{1}\left[P_{l}^{m-1}(x)\right]^{2} d x
$$

In other words,

$$
N_{l, m}=(l+m)(l-m+1) N_{l, m-1}
$$

and if we apply the same procedure $m$ times, this becomes

$$
\begin{aligned}
N_{l, m} & =(l+m)(l-m+1)(l+m-1)(l-m+2) N_{l, m-2} \\
& =\ldots= \\
& =(l+m)(l+m+1) \ldots(l+1) l \ldots(l-m+2)(l-m+1) N_{l, 0} \\
& =\frac{(l+m) !}{(l-m) !} \int_{-1}^{1}\left[P_{l}(x)\right]^{2} d x .
\end{aligned}
$$

But the latter integral is just the normalization integral for the Legendre polynomials and is given in (11.2.31). Therefore, our final result, combining the statement of orthogonality and normalization, is

$$
\int_{-1}^{1} P_{l}^{m}(x) P_{k}^{m}(x) d x=\frac{2}{2 l+1} \frac{(l+m) !}{(l-m) !} \delta_{l, k} .
$$

The statement of completeness is

$$
\int_{-1}^{1}[f(x)]^{2} d x=\sum_{l=m}^{\infty} \frac{2}{2 l+1} \frac{(l+m) !}{(l-m) !}\left[c_{l}^{m}\right]^{2}
$$


where

$$
c_{l}^{m}=\frac{2 l+1}{2} \frac{(l-m) !}{(l+m) !} \int_{-1}^{1} P_{l}^{m}(x) f(x) d x,
$$

$m$ is a fixed positive integer, and $f(x)$ is any function that is square integrable over the interval $-1 \leq x \leq 1$. Seen in isolation, this result does not appear to add a significant new tool to our box of problem solving techniques. After all, no one would choose to expand functions in terms of associated Legendre functions rather than the much simpler Legendre polynomials unless, of course, some other aspect of the problem gives rise to a compelling reason. This is exactly what happens in problems with spherical but not azimuthal symmetry and it is of such importance that we will devote a separate sub-section to it.

\subsubsection{Completeness and the Spherical Harmonics}

Separation of variables using spherical coordinates has taught us that any problem with spherical symmetry has a solution whose angular dependence can be expressed in terms of the solutions of the eigenvalue problem

$$
\frac{1}{\sin \theta} \frac{\partial}{\partial \theta}\left(\sin \theta \frac{\partial Y}{\partial \theta}\right)+\frac{1}{\sin ^{2} \theta} \frac{\partial^{2} Y}{\partial \varphi^{2}}=-\lambda Y(\theta, \varphi)
$$

where $Y(\theta, \varphi)$ is required to be single-valued and finite over (the sphere) $0 \leq \varphi \leq 2 \pi$, $-1 \leq \cos \theta \leq 1$. These solutions are

$$
Y(\theta, \varphi)=P_{l}^{m}(\cos \theta) e^{ \pm i m \theta}, \quad m=0,1,2, \ldots, l
$$

corresponding to eigenvalues $\lambda=l(l+1), l=0,1,2, \ldots$ Rather than carry the plusminus sign in the exponent, we shall allow $m$ to assume both positive and negative values. A further convenience is provided by inclusion of normalizing factors and a phase that is useful in quantum mechanical applications. Making all of these modifications, we obtain

$$
\begin{array}{r}
Y_{l}^{m}(\theta, \varphi)=(-1)^{m} \sqrt{\frac{2 l+1}{4 \pi} \frac{(l-m) !}{(l+m) !}} P_{l}^{m}(\cos \theta) e^{i m \varphi}, \quad l=0,1,2, \ldots, \\
m=-l,-l+1, \ldots, 0, \ldots, l-1, l .
\end{array}
$$

This set of functions is called spherical harmonics.

Notice that

$$
\left(Y_{l}^{m}(\theta, \varphi)\right)^{\star}=(-1)^{m} Y_{l}^{-m}(\theta, \varphi),
$$

and

$$
\int_{0}^{2 \pi} \int_{0}^{\pi}\left(Y_{l}^{m}(\theta, \varphi)\right)^{\star} Y_{k}^{n}(\theta, \varphi) \sin \theta d \theta d \varphi=\delta_{l, k} \delta_{m, n} .
$$




\begin{tabular}{lccc}
\hline & $m=0$ & $m=1$ & $m=2$ \\
$l=0$ & $\frac{1}{\sqrt{4 \pi}}$ & & \\
$l=1$ & $\sqrt{\frac{3}{4 \pi}} \cos \theta$ & $\sqrt{\frac{3}{8 \pi}} \sin \theta e^{i \varphi}$ & \\
$l=2$ & $\sqrt{\frac{5}{4 \pi}}\left(\frac{3}{2}\right)$ & $-\frac{1}{4} \sqrt{\frac{30}{\pi}} \cos \theta \sin \theta e^{i \varphi}$ & $\frac{1}{8} \sqrt{\frac{30}{\pi}} \sin ^{2} \theta e^{2 i \varphi}$ \\
\hline
\end{tabular}

Since for each value of $l$ there are $2 l+1$ allowed values of $m$, each eigenvalue $\lambda=l(l+1)$ corresponds to $2 l+1$ eigenfunctions $Y_{l}^{m}(\theta, \varphi)$ and so we say that it is $(2 l+1)$ - fold degenerate.

As eigenfunctions, the spherical harmonics are complete with respect to the space of square-integrable functions defined on the surface of a sphere. Thus, any function $f(\theta . \varphi)$ that is square integrable over the sphere can be represented by the convergent series

$$
f(\theta, \varphi)=\sum_{l=0}^{\infty} \sum_{m=-l}^{l} c_{l, m} Y_{l}^{m}(\theta, \varphi)
$$

where the coefficients are given by

$$
c_{l, m}=\int_{0}^{2 \pi} \int_{0}^{\pi}\left(Y_{l}^{m}(\theta, \varphi)\right)^{\star} f(\theta, \varphi) \sin \theta d \theta d \varphi .
$$

The closure relation for spherical harmonics is

$$
\sum_{l=0}^{\infty} \sum_{m=-l}^{l} Y_{l}^{m}(\theta, \varphi)\left(Y_{l}^{m}\left(\theta^{\prime}, \varphi^{\prime}\right)\right)^{\star}=\frac{\delta\left(\theta-\theta^{\prime}\right) \delta\left(\varphi-\varphi^{\prime}\right)}{\sin \theta} .
$$

We conclude the formal discussion of spherical harmonics by stating a fortuitous theorem that separates a dependence on the angle between two directions into a dependence on the directions themselves. Suppose that we have two coordinate vectors $\boldsymbol{r}$ and $\boldsymbol{r}^{\prime}$ with spherical coordinates $(r, \theta, \varphi)$ and $\left(r^{\prime}, \theta^{\prime}, \varphi^{\prime}\right)$, respectively. Trigonometry determines the cosine of the angle $\alpha$ between the two vectors to be

$$
\cos \alpha=\cos \theta \cos \theta^{\prime}+\sin \theta \sin \theta^{\prime} \cos \left(\varphi-\varphi^{\prime}\right) .
$$

Remarkably, when $\cos \alpha$ becomes the argument of a Legendre polynomial, the dependence on $\theta$ and $\varphi$ separates totally from that on $\theta^{\prime}$ and $\varphi^{\prime}$. In fact, what happens is

$$
P_{l}(\cos \alpha)=\frac{4 \pi}{2 l+1} \sum_{m=-l}^{l}\left(Y_{l}^{m}\left(\theta^{\prime}, \varphi^{\prime}\right)\right)^{\star} Y_{l}^{m}(\theta, \varphi) .
$$

This identity is known as the addition theorem. In the special case of $\alpha=0$, it produces the sum rule

$$
\sum_{m=-l}^{l}\left|Y_{l}^{m}(\theta, \varphi)\right|^{2}=\frac{2 l+1}{4 \pi}
$$




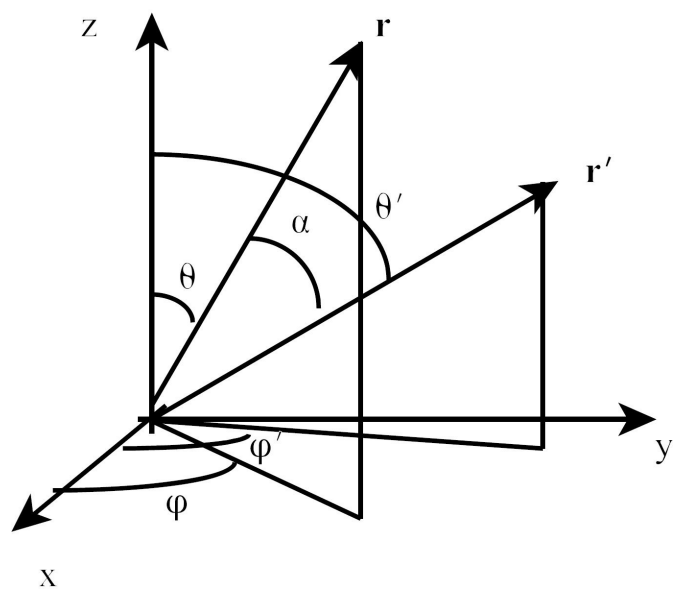

A far more important application, however, is in the expression of the inverse distance $\frac{1}{\left|\boldsymbol{r}-\boldsymbol{r}^{\prime}\right|}$ between two points in terms of spherical harmonics. This arises in a wide range of potential problems, from electromagnetic and gravitational theory to quantum mechanics. A further application of some importance involves the rotation of coordinate axes.

\subsubsection{Applications: Problems Without Azimuthal Symmetry}

We begin by pursuing the observation about the inverse distance between two points. The electrostatic potential at $r$ due to a point charge $q$ at $r^{\prime}$ is

$$
\psi(\boldsymbol{r})=\frac{1}{4 \pi \epsilon_{0}} \frac{q}{\left|\boldsymbol{r}-\boldsymbol{r}^{\prime}\right|} .
$$

If instead of a point charge we have a distribution with charge density $\rho\left(\boldsymbol{r}^{\prime}\right)$ confined to a region $r^{\prime}<R$, the electrostatic potential at $r, r>R$ is

$$
\psi(\boldsymbol{r})=\frac{1}{4 \pi \epsilon_{0}} \int_{V} \frac{\rho\left(\boldsymbol{r}^{\prime}\right)}{\left|\boldsymbol{r}-\boldsymbol{r}^{\prime}\right|} d^{3} \boldsymbol{r}^{\prime}
$$

In either case, knowledge of the inverse distance $\frac{1}{\left|\boldsymbol{r}-\boldsymbol{r}^{\prime}\right|}$ is critical.

The generating function for Legendre polynomials is

$$
\frac{1}{\sqrt{1-2 x t+t^{2}}}=\sum_{l=0}^{\infty} t^{l} P_{l}(x), \quad|t|<1
$$

and the inverse distance can be cast into exactly this form:

$$
\frac{1}{\left|\boldsymbol{r}-\boldsymbol{r}^{\prime}\right|}=\frac{1}{\sqrt{\left(\boldsymbol{r}-\boldsymbol{r}^{\prime}\right)^{2}}}=\frac{1}{\sqrt{r^{2}-2 r r^{\prime} \cos \alpha+r^{\prime 2}}}=\frac{1}{r \sqrt{1-2 \frac{r^{\prime}}{r} \cos \alpha+\left(\frac{r^{\prime}}{r}\right)^{2}}} .
$$


Thus, identifying $\cos \alpha$ with $x$ and $\frac{r^{\prime}}{r}$ with $t$, we have

$$
\frac{1}{\left|\boldsymbol{r}-\boldsymbol{r}^{\prime}\right|}=\sum_{l=0}^{\infty} \frac{r^{\prime l}}{r^{l+1}} P_{l}(\cos \alpha), \quad r^{\prime}<r .
$$

Invoking the addition theorem, this becomes the fully separated expression

$$
\frac{1}{\left|\boldsymbol{r}-\boldsymbol{r}^{\prime}\right|}=\sum_{l=0}^{\infty} \sum_{m=-l}^{l} \frac{4 \pi}{2 l+1} r^{\prime l}\left(Y_{l}^{m}\left(\theta^{\prime}, \varphi^{\prime}\right)\right)^{*} \frac{1}{r^{l+1}} Y_{l}^{m}(\theta, \varphi)
$$

which, when substituted into equation (11.2.61), produces a representation of the potential due to a distributed charge that has an immediate physical interpretation. The representation is

$$
\psi(\boldsymbol{r})=\frac{1}{\epsilon_{0}} \sum_{l=0}^{\infty} \sum_{m=-l}^{l} \frac{1}{2 l+1} \frac{Q_{l}^{m}}{r^{l+1}} Y_{l}^{m}(\theta, \varphi)
$$

where

$$
Q_{l}^{m}=\int_{V}\left(Y_{l}^{m}\left(\theta^{\prime}, \varphi^{\prime}\right)\right)^{\star} r^{\prime l} \rho\left(\boldsymbol{r}^{\prime}\right) d^{3} \boldsymbol{r}^{\prime}
$$

Evidently, the $Q_{l}^{m}$ are spherical components of the multipole moments of the charge distribution. Therefore, the representation of $\psi(\boldsymbol{r})$ in terms of them is called a multipole expansion. Notice that each term has a distinctive angular distribution and an inverse dependence on $r$ that falls off more rapidly with increasing $l$. The lowest order multipole moments are the monopole,

$$
Q_{0}^{0}=\frac{1}{\sqrt{4 \pi}} \int_{V} \rho\left(\boldsymbol{r}^{\prime}\right) d^{3} \boldsymbol{r}^{\prime}=\frac{1}{\sqrt{4 \pi}} q=\frac{1}{\sqrt{4 \pi}} \times(\text { the total charge present }),
$$

the dipole,

$$
Q_{1}^{ \pm 1}=\mp \sqrt{\frac{3}{8 \pi}}\left(p_{1} \mp i p_{2}\right), Q_{1}^{0}=\sqrt{\frac{3}{4 \pi}} p_{3} \text { where } p=\int_{V} r^{\prime} \rho\left(\boldsymbol{r}^{\prime}\right) d^{3} \boldsymbol{r}^{\prime},
$$

and the quadrupole,

$$
\begin{aligned}
Q_{2}^{ \pm 2} & =\frac{1}{12} \sqrt{\frac{15}{2 \pi}}\left(Q_{11} \mp 2 i Q_{12}-Q_{22}\right), \quad Q_{2}^{ \pm 1}=\mp \frac{1}{3} \sqrt{\frac{15}{4 \pi}}\left(Q_{13} \mp i Q_{23}\right), \\
Q_{2}^{0} & =\frac{1}{2} \sqrt{\frac{5}{4 \pi}} Q_{33} \text { where } Q_{i j}=\int_{V}\left(3 x_{i}^{\prime} x_{j}^{\prime}-r^{\prime 2} \delta_{i j}\right) \rho\left(\boldsymbol{r}^{\prime}\right) d^{3} \boldsymbol{r}^{\prime} .
\end{aligned}
$$

Thus, with respect to a Cartesian basis, our multipole expansion is

$$
\psi(\boldsymbol{r})=\frac{1}{4 \pi \epsilon_{0}}\left\{\frac{q}{r}+\frac{\boldsymbol{p} \cdot \boldsymbol{r}}{r^{3}}+\frac{1}{2} \sum_{i, j=1}^{3} Q_{i j} \frac{x_{i} x_{j}}{r^{5}}+\ldots\right\}
$$


Evidently an expansion of a potential in terms of spherical harmonics is effectively the same as an expansion of the source charge distribution in terms of its multipole components. To reinforce our intuitive appreciation of this, we shall determine the potential due to specific multipole configurations of discrete charges.

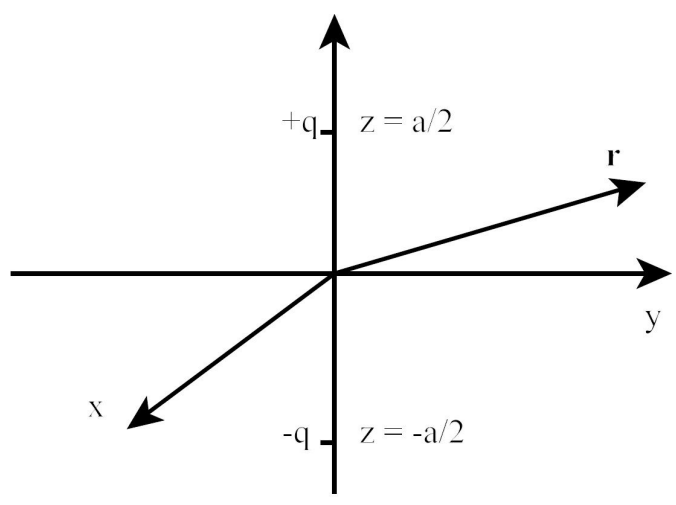

We start with a dipole constructed from a charge $q$ located at $z=\frac{a}{2}$ and charge $-q$ at $z=-\frac{a}{2}$. The resulting potential at a point $r$ is

$$
\begin{aligned}
\psi(\boldsymbol{r}) & =\frac{q}{4 \pi \epsilon_{0}}\left[\frac{1}{\left|r-\frac{a}{2} k\right|}-\frac{1}{\left|r+\frac{a}{2} k\right|}\right] \\
& =\frac{q}{4 \pi \epsilon_{0}} \frac{1}{r}\left[\left\{1-2 \frac{a}{2 r} \cos \theta+\left(\frac{a}{2 r}\right)^{2}\right\}^{-\frac{1}{2}}-\left\{1+2 \frac{a}{2 r} \cos \theta+\left(\frac{a}{2 r}\right)^{2}\right\}^{-\frac{1}{2}}\right]
\end{aligned}
$$

or,

$$
\begin{aligned}
\psi(\boldsymbol{r}) & =\frac{q}{4 \pi \epsilon_{0}} \frac{1}{r}\left[\sum_{l=0}^{\infty}\left(\frac{a}{2 r}\right)^{l} P_{l}(\cos \theta)-\sum_{l=0}^{\infty}\left(\frac{-a}{2 r}\right)^{l} P_{l}(\cos \theta)\right] \\
& =\frac{2 q}{4 \pi \epsilon_{0}} \frac{1}{r}\left[\left(\frac{a}{2 r}\right) P_{1}(\cos \theta)+\left(\frac{a}{2 r}\right)^{3} P_{3}(\cos \theta)+\ldots\right]
\end{aligned}
$$

Thus, at a distant point, $r \gg a$, the potential is

$$
\psi(\boldsymbol{r}) \simeq \frac{q a}{4 \pi \epsilon_{0}} \frac{P_{1}(\cos \theta)}{r^{2}}=\frac{1}{4 \pi \epsilon_{0}} \frac{q \operatorname{arcos} \theta}{r^{3}}=\frac{1}{4 \pi \epsilon_{0}} \frac{\boldsymbol{p} \cdot \boldsymbol{r}}{r^{3}}
$$

where $\boldsymbol{p}=q a \boldsymbol{k}$ is the dipole moment that we learned to associate with such a charge distribution in introductory electricity and magnetism.

We shall now move the dipole off the $\mathrm{z}$-axis so that it can be combined with a second dipole to form an electric quadrupole. To be as general as possible, we will use an arbitrary azimuthal orientation and locate charge $\mathrm{q}$ at $\boldsymbol{r}^{\prime}=\left(\frac{a}{\sqrt{2}}, \frac{\pi}{4}, \varphi^{\prime}\right)$ and $-q$ 
at $\boldsymbol{r}^{\prime \prime}=\left(\frac{a}{\sqrt{2}}, \frac{3 \pi}{4}, \varphi^{\prime}\right)$. Again, we have

$$
\psi(\boldsymbol{r})=\frac{q}{4 \pi \epsilon_{0}}\left[\frac{1}{\left|\boldsymbol{r}-\boldsymbol{r}^{\prime}\right|}-\frac{1}{\left|\boldsymbol{r}-\boldsymbol{r}^{\prime \prime}\right|}\right]
$$

but this time we have to use the addition theorem in the form

$$
\frac{1}{\left|\boldsymbol{r}-\boldsymbol{r}^{\prime}\right|}=\sum_{l=0}^{\infty} \sum_{m=-l}^{l} \frac{4 \pi}{2 l+1} \frac{r^{\prime l}}{r^{l+1}}\left(Y_{l}^{m}\left(\theta^{\prime}, \varphi^{\prime}\right)\right)^{\star} Y_{l}^{m}(\theta, \varphi), \quad r>r^{\prime}
$$

to expand the two inverse distances. Then, since $\cos \frac{3 \pi}{4}=-\cos \frac{\pi}{4}$, we have $P_{l}^{m}\left(\cos \frac{3 \pi}{4}\right)=$ $P_{l}^{m}\left(-\cos \frac{\pi}{4}\right)=(-1)^{l+m} P_{l}^{m}\left(\cos \frac{\pi}{4}\right)$ and hence,

$$
\psi(\boldsymbol{r})=\frac{q}{4 \pi \epsilon_{0}} \sum_{l=0}^{\infty} \sum_{m=-l}^{l} \frac{4 \pi}{2 l+1} \frac{a^{l}}{2^{l / 2} r^{l+1}}\left[1-(-1)^{l+m}\right]\left(Y_{l}^{m}\left(\frac{\pi}{4}, \varphi^{\prime}\right)\right)^{\star} Y_{l}^{m}(\theta, \varphi) .
$$

The factor $\varepsilon_{l, m}=1-(-1)^{l+m}$ vanishes for even values of $l+m$. Therefore, we find $\psi(\boldsymbol{r})=\frac{q}{\epsilon_{0}}\left[\frac{1}{3 \sqrt{2}} \frac{a}{r^{2}} Y_{1}^{0}\left(\frac{\pi}{4}, \varphi^{\prime}\right) Y_{1}^{0}(\theta, \varphi)+\frac{1}{5} \frac{a^{2}}{r^{3}} \operatorname{Re}\left[\left(Y_{2}^{1}\left(\frac{\pi}{4}, \varphi^{\prime}\right)\right)^{\star} Y_{2}^{1}(\theta, \varphi)\right]+\mathrm{O}\left(\frac{a^{3}}{r^{4}}\right)\right]$ or, for $r \ll a$

$$
\psi(\boldsymbol{r}) \simeq \frac{\sqrt{2} q a}{4 \pi \epsilon_{0}} \frac{1}{r^{2}} P_{1}\left(\cos \frac{\pi}{4}\right) P_{1}(\cos \theta)=\frac{q a}{4 \pi \epsilon_{0}} \frac{\cos \theta}{r^{2}}
$$

As we would expect, this is the same as the result obtained for a dipole on the $z$-axis. A dependence on the azimuthal angles enters the picture only when the relative size of $r$ and $a$ justifies inclusion of the $\frac{a^{2}}{r^{3}}$ term. However, as we shall now see this becomes the leading term when we combine two dipoles to form a quadrupole.

The quadrupole is constructed by placing a second dipole a distance $a$ from the first and with its charges oriented so that the dipoles are anti-parallel. So, if the first is at an azimuthal angle $\varphi^{\prime}$, the second is at $\varphi^{\prime}+\pi$. Therefore, since

$$
e^{i m\left(\varphi^{\prime}+\pi\right)}=e^{i m \pi} e^{i m \varphi^{\prime}}=(-1)^{m} e^{i m \varphi^{\prime}},
$$

the potential due to the two dipoles is

$\psi(\boldsymbol{r})=\frac{q}{4 \pi \epsilon_{0}} \sum_{l=0}^{\infty} \sum_{m=-l}^{l} \frac{4 \pi}{2 l+1} \frac{a^{l}}{2^{l / 2} r^{l+1}}\left[1-(-1)^{l+m}\right]\left[1-(-1)^{m}\right]\left(Y_{l}^{m}\left(\frac{\pi}{4}, \varphi^{\prime}\right)\right)^{\star} Y_{l}^{m}(\theta, \varphi)$.

The numerical factor $\chi_{l, m}=\left[1-(-1)^{l+m}\right]\left[1-(-1)^{m}\right]$ results in all the lowest order terms vanishing with the first non-zero term corresponding to $l=2, m= \pm 1$ and the next to $l=4, m= \pm 3$ and \pm 1 . Thus, for $r \gg a$

$$
\psi(\boldsymbol{r}) \simeq \frac{q}{4 \pi \epsilon_{0}} \frac{4 \pi}{5} \frac{a^{2}}{2 r^{3}} 8 \operatorname{Re}\left[\left(Y_{2}^{1}\left(\frac{\pi}{4}, \varphi^{\prime}\right)\right)^{\star} Y_{2}^{1}(\theta, \varphi)\right]=\frac{3 q}{4 \pi \varepsilon_{0}} \frac{a^{2}}{r^{3}} \sin \theta \cos \theta \cos \left(\varphi-\varphi^{\prime}\right) .
$$


An electric octupole can similarly be constructed from two quadrupoles and one finds that a further cancellation occurs leaving the $l=3$ term as the leading one in the spherical harmonic expansion of its potential. Generalizing, we can assert that the potential due to an electric $2^{l}$-pole falls off like $r^{-l}$ and has an angular dependence determined by $Y_{l}^{m}(\theta, \varphi)$ for $r \gg a$.

We now turn our attention to solving problems involving Laplace's equation and non-homogeneous boundary conditions. Suppose that we have a spherical shell of radius $R$ which is maintained at a potential $V_{0} \cos 2 \varphi$. Let us find the potential at any point inside the sphere.

This is an interior problem and so the solution must have the characteristic $r$ -dependence that ensures boundedness at the origin. However, unlike the interior problem of Section 11.2.5, this one manifestly lacks azimuthal symmetry. Therefore, rather than use a simple Fourier- Legendre representation of the potential, we now must work with the spherical harmonic expansion

$$
\psi(r, \theta, \varphi)=\sum_{l=0}^{\infty} \sum_{m=-l}^{l} A_{l m} r^{l} Y_{l}^{m}(\theta, \varphi) .
$$

(This problem is more amenable to use of the equivalent explicitly real expansion

$$
\psi(r, \theta, \varphi)=\sum_{l=0}^{\infty} \sum_{m=0}^{l} r^{l} P_{l}^{m}(\cos \theta)\left(a_{l m} \cos m \varphi+b_{m l} \sin m \varphi\right) .
$$

However, the object of the exercise is to gain experience working with spherical harmonics.)

Using (11.2.56) and the boundary condition $\psi(R, \theta, \varphi)=V_{0} \cos 2 \varphi$, we have

$$
A_{l m}=\frac{V_{0}}{R^{l}} \int_{0}^{2 \pi} \int_{0}^{\pi}\left(Y_{l}^{m}(\theta, \varphi)\right)^{\star} \cos 2 \varphi \sin \theta d \theta d \varphi
$$

or,

$$
A_{l m}=\frac{V_{0}}{R^{l}} \sqrt{\frac{2 l+1)}{4 \pi} \frac{(l-|m|) !}{(l+|m|) !}} \int_{0}^{2 \pi} \int_{0}^{\pi} P_{l}^{|m|}(\cos \theta) e^{-i m \varphi} \cos 2 \varphi \sin \theta d \theta d \varphi .
$$

The integration over $\varphi$ gives us

$$
\int_{0}^{2 \pi} e^{-i m \varphi} \cos 2 \varphi d \varphi=\frac{1}{2} \int_{0}^{2 \pi} e^{-i m \varphi}\left(e^{2 i \varphi}+e^{-2 i \varphi}\right) d \varphi= \begin{cases}0 & \text { if } m \neq \pm 2 \\ \pi & \text { if } m= \pm 2\end{cases}
$$

This means, of course, that in addition to the restriction on $m$, we must restrict $l$ to $l \geq 2$. Proceeding with the $\theta$ integration, we have

$$
\int_{0}^{2 \pi} P_{l}^{2}(\cos \theta) \sin \theta d \theta=\int_{-1}^{1} P_{l}^{2}(x) d x=\int_{-1}^{1}\left(1-x^{2}\right) \frac{d^{2} P_{l}}{d x^{2}} d x
$$


where we have made the substitution $x=\cos \theta$. Integrating twice by parts, we find

$$
\int_{-1}^{1}\left(1-x^{2}\right) \frac{d^{2} P_{l}}{d x^{2}} d x=\left.2 x P_{l}(x)\right|_{-1} ^{1}-2 \int_{-1}^{1} P_{l}(x) d x=2\left[1+(-1)^{l}\right]-2 \int_{-1}^{1} P_{l}(x) P_{0}(x) d x .
$$

The last integral is zero because $l \geq 2$ and the Legendre polynomials form an orthogonal set. Therefore,

$$
\int_{0}^{\pi} P_{l}^{2}(\cos \theta) \sin \theta d \theta= \begin{cases}0 & \text { if } l \text { is odd } \\ 4 & \text { if } l \text { is even }\end{cases}
$$

Collecting all this information, we conclude that

$$
\begin{aligned}
\psi(r, \theta, \varphi) & =\sum_{l=2,4, \ldots}^{\infty} \frac{4 \pi V_{0}}{R^{l}} \sqrt{\frac{(2 l+1)}{4 \pi} \frac{(l-2) !}{(l+2) !}} r^{l}\left[Y_{l}^{2}(\theta, \varphi)+Y_{l}^{-2}(\theta, \varphi)\right] \\
& =\sum_{l=2,4, \ldots}^{\infty} 2 V_{0}(2 l+1) \frac{(l-2) !}{(l+2) !}\left(\frac{r}{R}\right)^{l} P_{l}^{2}(\cos \theta) \cos 2 \varphi .
\end{aligned}
$$

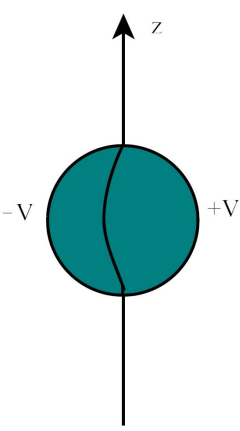

As a further application, consider two hemispherical shells of radius $b$ separated by a thin ring of insulation and maintained at potentials $V$ and $-V$ as shown in the diagram above. The boundary condition at the surface of the sphere is

$$
\psi(b, \theta, \varphi)= \begin{cases}+V & \text { if } 0 \leq \varphi<\pi \\ -V & \text { if } \pi<\varphi \leq 2 \pi\end{cases}
$$

which is certainly not azimuthally symmetric. Therefore, if we wish to find the potential at any point inside the sphere, we will have to resort a second time to the expansion

$$
\psi(r, \theta, \varphi)=\sum_{l=0}^{\infty} \sum_{m=-l}^{l} A_{l m} r^{l} Y_{l}^{m}(\theta, \varphi)
$$


Using (11.2.56) plus our boundary condition at $r=b$, we determine the coefficients $A_{l m}$ to be

$$
\begin{aligned}
& A_{l m}=\frac{1}{b^{l}} \int_{-1}^{1} \int_{0}^{2 \pi}\left(Y_{l}^{m}(\theta, \varphi)\right)^{\star} \psi(b, \theta, \varphi) d(\cos \theta) d \varphi \\
& =\frac{V}{b^{l}} \sqrt{\frac{2 l+1}{4 \pi} \frac{(l-m) !}{(l+m) !}} \int_{-1}^{1} P_{l}^{m}(\cos \theta) d(\cos \theta)\left[\int_{0}^{\pi} e^{-i m \varphi} d \varphi-\int_{\pi}^{2 \pi} e^{-i m \varphi} d \varphi\right]
\end{aligned}
$$

The factor in square brackets is

$$
\int_{0}^{\pi} e^{-i m \varphi} d \varphi-\int_{\pi}^{2 \pi} e^{-i m \varphi} d \varphi=\frac{2 i}{m}\left[(-1)^{m}-1\right] .
$$

Thus, $m$ can only assume odd integer values. Recalling the parity $(-1)^{l+m}$ of associated Legendre functions, we realize that this means that $l$ must also be restricted to odd values. To evaluate the integral over $\cos \theta$ we can either resort to integral tables or we can evaluate term by term starting from $l=1, m=1$ and try to deduce an expression for the general term by interpolation. The first option sounds simple until we discover that this is an uncommon integral and when listed, it is expressed in terms of hypergeometric series. The second option sounds tedious at best and at worst an opportunity to commit egregious arithmetical errors. And so, we search for a third option.

Comparing the diagram for this problem with that for the hemispherical shells in Section 11.2.5, we realize that this is the same problem but with either the sphere or the coordinate axes rotated through $90^{\circ}$. Therefore, we can write down the solution. It is

$$
\psi(r, \theta, \varphi)=V \sum_{l=1,3,5, \ldots}^{\infty} \frac{(2 l+1)(-1)^{\frac{l-1}{2}}(l+1) !}{2^{l+1}\left(\frac{l+1}{2}\right)^{2} l}\left(\frac{r}{b}\right)^{l} P_{l}(\cos \alpha),
$$

but the angle $\alpha$ that appears here is not the polar angle but rather the angle between $\boldsymbol{r}$ and the $y$-axis. Nevertheless, this is a major breakthrough because we can use the addition theorem to relate $P_{l}(\cos \alpha)$ to spherical harmonics in the angular coordinates of $\boldsymbol{r}$ and of the $y$-axis. In fact, from (11.2.59) we have

$$
P_{l}(\cos \alpha)=\frac{4 \pi}{2 l+1} \sum_{m=-l}^{l}\left(Y_{l}^{m}\left(\frac{\pi}{2}, \frac{\pi}{2}\right)\right)^{\star} Y_{l}^{m}(\theta, \varphi) .
$$

Since $\cos \frac{\pi}{2}=0$, this becomes 


$$
\begin{aligned}
P_{l}(\cos \alpha) & =\sum_{m=-l}^{l} \frac{(l-|m|) !}{(l+m) !} P_{l}^{|m|}(0) e^{-i m \frac{\pi}{2}} P_{l}^{|m|}(\cos \theta) e^{i m \varphi} \\
& =2 \sum_{m=0}^{l} \frac{(l-m) !}{(l+m) !} P_{l}^{m}(0) P_{l}^{m}(\cos \theta) \sin m \varphi .
\end{aligned}
$$

The special value $P_{l}^{m}(0)$ can be worked out and one finds

$$
P_{l}^{m}(0)=\frac{2^{m} \sqrt{\pi}}{\Gamma\left(\frac{l-m}{2}+1\right) \Gamma\left(\frac{-l-m+1}{2}\right)} .
$$

In our case, both $l$ and $m$ are odd. Therefore, the first gamma function in the denominator can be replaced by $\left(\frac{l-m}{2}\right) !=\frac{(l-m) ! !}{2^{\frac{l-m}{2}}}$ and the second by $\frac{\sqrt{\pi}(-1)^{\frac{l+m}{2}} 2^{\frac{l+m}{2}}}{(l+m-1) ! !}$. Collecting all this information and substituting into the expression for the potential, we conclude that

$$
\psi(\boldsymbol{r})=V \sum_{l=\text { odd }}^{\infty} \sum_{m=\text { odd }}^{l} \frac{(2 l+1)(l+1) !}{2^{l}\left(\frac{l+1}{2} !\right)^{2} l}(-1)^{\frac{m+1}{2}} \frac{(l-m-1) ! !}{(l+m) ! !}\left(\frac{r}{b}\right)^{l} P_{l}(\cos \theta) \sin m \varphi
$$

where (-1)!! and (0)!! are both understood to be 1 .

\subsection{Bessel Functions: Problems Possessing Cylindrical Symmetry}

\subsubsection{Properties of Bessel and Neumann Functions}

As we saw in Section 10.4, separation of variables applied to the Helmholtz and Laplace's equation when cylindrical coordinates are used results in a radial equation that can be transformed into Bessel's DE

$$
\frac{d^{2} R}{d \rho^{2}}+\frac{1}{\rho} \frac{d R}{d \rho}+\left(1-\frac{m^{2}}{\rho^{2}}\right) R=0, \quad m=0,1,2, \ldots
$$

by the simple expedient of replacing the radial variable $r$ by $\rho=\alpha r$ where $\alpha^{2}=k^{2}-\lambda_{2}$, $k^{2}$ is the Helmholtz equation parameter and $\lambda_{2}$ is the separation constant associated with the $z$-dependence. The parameter $m^{2}$ is the separation constant associated with the $\theta$-dependence and was determined by imposition of the homogeneous boundary condition that we have solutions that are single-valued functions of $\theta$. The general solution of (11.3.1) is the linear combination

$$
R(r)=c_{1} J_{m}(\alpha r)+c_{2} N_{m}(\alpha r)
$$


where $J_{m}(x)$ and $N_{m}(x)$ are the Bessel and Neumann functions of order $m$, respectively. We solved for them explicitly in Chapter 9 and found the series representations (9.7.11) and (9.7.33). What we need to do now is relate those of their properties that are most germane to the solution of boundary value problems.

The Bessel functions are well behaved both at the origin and as $x \rightarrow \infty$. In fact, they "look" like lightly damped sine or cosine functions. Like sines and cosines, they are oscillatory functions with infinitely many zeros. However, the Bessel function zeros are not equally spaced. With the sole exception of $J_{0}(x)$ all of the $J_{m}(x)$ are zero at $x=0$. Moreover, using the first term in the power series (9.7.11), we see that

$$
J_{m}(x) \simeq \frac{1}{\Gamma(m+1)}\left(\frac{x}{2}\right)^{m} \text { as } x \rightarrow 0
$$

The Neumann functions are not well behaved at $x=0 . N_{0}(z)$ has a logarithmic branch point there and $N_{m}(z), m>0$, has a pole of order $m$. Thus,

$$
N_{0}(x) \simeq \frac{2}{\pi} \ln x \text { as } x \rightarrow 0,
$$

and

$$
N_{m}(x) \simeq-\frac{(m-1) !}{\pi}\left(\frac{2}{x}\right)^{m} \text { as } x \rightarrow 0 .
$$

The asymptotic or large $x$ behaviour of the Bessel and Neumann functions can be deduced from integral representations and the method of steepest descents; (see Section 6.3). One finds for $x \gg m$

$$
J_{m}(x) \sim \sqrt{\frac{2}{\pi x}} \cos \left(x-\frac{m \pi}{2}-\frac{\pi}{4}\right)
$$

and

$$
N_{m}(x) \sim \sqrt{\frac{2}{\pi x}} \sin \left(x-\frac{m \pi}{2}-\frac{\pi}{4}\right) .
$$

The complementarity of these two expressions reflects the judiciousness of the choice made for the definition of the Neumann functions.

Notice that the linear combinations

$$
H_{m}^{(1)}(x)=J_{m}(x)+i N_{m}(x) \text { and } H_{m}^{(2)}(x)=J_{m}(x)-i N_{m}(x)
$$

have the asymptotic forms

$$
H_{m}^{(1)}(x) \sim \sqrt{\frac{2}{\pi x}} \exp \left[i\left(x-\frac{m \pi}{2}-\frac{\pi}{4}\right)\right]
$$

and

$$
H_{m}^{(2)}(x) \sim \sqrt{\frac{2}{\pi x}} \exp \left[-i\left(x-\frac{m \pi}{2}-\frac{\pi}{4}\right)\right] .
$$


These are called Hankel functions or Bessel functions of the third kind.

Hankel functions are named for the complex analyst Hermann Hankel (1839-1873). He worked with a who's who of nineteenth century German mathematicians including Kronecker, Möbius, Riemann and Weierstrass.

Recurrence relations for the Bessel functions can be derived directly from their power series representation (9.7.11). Dividing it by $x^{m}$ and then differentiating, we find

$$
\frac{d}{d x}\left(\frac{J_{m}(x)}{x^{m}}\right)=\sum_{k=1}^{\infty} \frac{(-1)^{k}}{(k-1) !(k+m) !} \frac{x^{2 k-1}}{2^{2 k+m-1}} .
$$

This can be related to $J_{m+1}(x)$ by replacing $k$ by $k+1$ :

$$
\frac{d}{d x}\left(\frac{J_{m}(x)}{x^{m}}\right)=\sum_{k=0}^{\infty} \frac{(-1)^{k+1}}{k !(k+m+1) !} \frac{x^{2 k+1}}{2^{2 k+m+1}}=-\frac{J_{m+1}(x)}{x^{m}}
$$

which is valid for all $m \geq 0$. A particularly useful special case occurs for $m=0$ :

$$
\frac{d}{d x} J_{0}(x)=-J_{1}(x)
$$

Notice that repeated application of (11.3.11) , starting with the $m=0$ case, allows us to relate each $J_{m}(x)$ back to $J_{0}(x)$. In fact, we can write down a Rodrigues-like formula,

$$
J_{m}(x)=x^{m}\left(-\frac{1}{x} \frac{d}{d x}\right)^{m} J_{0}(x),
$$

which means that the differential operator $-\frac{1}{x} \frac{d}{d x}$ is to be applied $m$ times to $J_{0}(x)$ and the result is then multiplied by $x^{m}$.

Similarly, multiplying the power series for $J_{m}(x)$ by $x^{m}$ and differentiating, we have

$$
\frac{d}{d x}\left[x^{m} J_{m}(x)\right]=\sum_{k=0}^{\infty} \frac{(-1)^{k}}{k !(k+m-1) !} \frac{x^{2 k+2 m-1}}{2^{2 k+m-1}}=x^{m} J_{m-1}(x)
$$

which is valid for $m \geq 1$.

Adding $J_{m+1}(x)=-x^{m} \frac{d}{d x}\left(\frac{J_{m}(x)}{x^{m}}\right)$ to $J_{m-1}(x)=\frac{1}{x^{m}} \frac{d}{d x}\left[x^{m} J_{m}(x)\right]$, we establish the recurrence relation

$$
J_{m+1}(x)+J_{m-1}(x)=\frac{2 m}{x} J_{m}(x) .
$$

Subtracting them gives us

$$
J_{m+1}(x)-J_{m-1}(x)=-2 \frac{d J_{m}}{d x} .
$$

One can show that Bessel functions of integral order have a generating function of the form

$$
\exp \left[\frac{z}{2}\left(t-\frac{1}{t}\right)\right]=\sum_{m=-\infty}^{\infty} J_{m}(z) t^{m}, \quad t \neq 0
$$


where we have switched to a complex independent variable for reasons that will become obvious when we look at applications. The proof is straightforward. From the Taylor series representation of the exponential, we have

$$
\begin{aligned}
\exp \left[\frac{z}{2}\left(t-\frac{1}{t}\right)\right] & =\sum_{j=0}^{\infty} \frac{1}{j !}\left(\frac{z}{2}\right)^{j} t^{j} \cdot \sum_{k=0}^{\infty} \frac{(-1)^{k}}{k !}\left(\frac{z}{2}\right)^{k}\left(\frac{1}{t}\right)^{k} \\
& =\sum_{j=0}^{\infty} \sum_{k=0}^{\infty} \frac{(-1)^{k}}{j ! k !}\left(\frac{z}{2}\right)^{j+k} t^{j-k}
\end{aligned}
$$

All that remains is to replace the sum over $j$ by one over $m=j-k$ since that transforms our power series into

$$
\exp \left[\frac{z}{2}\left(t-\frac{1}{t}\right)\right]=\sum_{m=-\infty}^{\infty} \sum_{k=0}^{\infty} \frac{(-1)^{k}}{k !(k+m) !}\left(\frac{z}{2}\right)^{2 k+m} t^{m}=\sum_{m=-\infty}^{\infty} J_{m}(z) t^{m} .
$$

Notice that the generating function series is a Laurent rather than a Taylor series and that Laurent's theorem gives us an immediate contour integral representation of $J_{m}(z)$ :

$$
J_{m}(z)=\frac{1}{2 \pi i} \int_{C} t^{-m-1} e^{\frac{z}{2}\left(t-\frac{1}{t}\right)} d t
$$

where $C$ is a closed contour about the origin. Changing the integration variable to $u=$ $\frac{z t}{2}$ this becomes

$$
J_{m}(z)=\frac{1}{2 \pi i}\left(\frac{z}{2}\right)^{m} \int_{C} u^{-m-1} \exp \left(u-\frac{z^{2}}{4 u}\right) d u
$$

which we recognize as the integral representation (9.9.33) of Chapter 9.

A Fourier integral representation is obtained by setting $t=e^{i \theta}$. The generating function becomes

$$
e^{i z \sin \theta}=\sum_{m=-\infty}^{\infty} e^{i m \theta} J_{m}(z)
$$

with Fourier coefficients

$$
J_{m}(z)=\frac{1}{2 \pi} \int_{-\pi}^{\pi} e^{i z \sin \theta} e^{-i m \theta} d \theta
$$

Because Bessel's equation is of the Sturm-Liouville form, the radial equation (11.3.1)

$$
\frac{d^{2} R}{d \rho^{2}}+\frac{1}{\rho} \frac{d R}{d \rho}+\left(1-\frac{m^{2}}{\rho^{2}}\right) R=0, \quad \rho=\alpha r=\sqrt{k^{2}-\lambda_{2}} r
$$


or

$$
\frac{d}{d r}\left(r \frac{d R}{d r}\right)-\frac{m^{2}}{r} R(r)=-\alpha^{2} r R(r)
$$

becomes an eigenvalue equation with eigenvalue $\alpha^{2}$ as soon as we impose homogeneous boundary conditions on the general solution $R(r)=c_{1} J_{m}(\alpha r)+c_{2} N_{m}(\alpha r)$. Let us suppose that the range of $r$ is $0 \leq r \leq a$. Typically, one boundary condition will be that $R(r)$ be bounded at the origin: $|R(0)|<\infty$. This immediately eliminates the Neumann functions from consideration and we conclude that $R(r) \propto J_{m}(\alpha r)$. The second condition will most likely be

$$
\text { either } R(a)=0 \text { or }\left.\frac{d R}{d r}\right|_{r=a}=0
$$

which implies that

$$
\text { either } J_{m}(\alpha a)=0 \text { or } J_{m}^{\prime}(\alpha a)=0 \text {. }
$$

Denoting the nth zero of $J_{m}(x)$ by $x_{m, n}$ and the nth zero of $J_{m}^{\prime}(x)$ by $y_{m, n}$, we see that the eigenvalues are

$$
\text { either } \alpha_{m, n}^{2}=\frac{x_{m, n}^{2}}{a^{2}} \text { or } \alpha_{m, n}^{2}=\frac{y_{m, n}^{2}}{a^{2}}
$$

and the corresponding (unnormalized) eigenfunctions are

$$
R_{n}(r)=J_{m}\left(\alpha_{m, n} r\right), \quad n=1,2,3, \ldots
$$

These must comprise an orthogonal set with their orthogonality (with respect to weight function $w(r)=r$ ) expressed by

$$
\int_{0}^{a} R_{n}(r) R_{p}(r) r d r=\int_{0}^{a} J_{m}\left(\alpha_{m, n} r\right) J_{m}\left(\alpha_{m, p} r\right) r d r=0 \text { for } n \neq p .
$$

The zeros of $J_{m}(x)$ and $J_{m}^{\prime}(x)$ are tabulated in standard references such as Abramowicz and Stegun. For future convenience, we provide below a limited table containing the first four zeros of the first five Bessel functions.

$$
\begin{array}{ccccl} 
& n=1 & n=2 & n=3 & n=4 \\
m=0 & 2.404 & 5.520 & 8.654 & 11.792 \\
m=1 & 3.832 & 7.016 & 10.173 & 13.323 \\
m=2 & 5.135 & 8.417 & 11.620 & 14.796 \\
m=3 & 6.379 & 9.760 & 13.017 & 16.224 \\
m=4 & 7.586 & 11.064 & 14.373 & 17.616
\end{array}
$$


The determination of the norm of $J_{m}\left(\alpha_{m, n} r\right)$ requires evaluation of the integral

$$
N_{m, n}=\int_{0}^{a}\left[J_{m}\left(\alpha_{m, n} r\right)\right]^{2} r d r=\frac{1}{\alpha_{m, n}^{2}} \int_{0}^{\alpha_{m, n} a}\left[J_{m}(x)\right]^{2} x d x=\frac{1}{\alpha_{m, n}^{2}} I_{m, n}
$$

where we have set $x=\alpha_{m, n} r$. Integrating once by parts, we have

$$
I_{m, n}=\left.\frac{1}{2}\left[J_{m}(x)\right]^{2}\right|_{x=0} ^{x=\alpha_{m, n} a}-\int_{0}^{\alpha_{m, n} a} J_{m}(x) J_{m}^{\prime}(x) x^{2} d x .
$$

But, from Bessel's equation we have

$$
x^{2} J_{m}(x)=m^{2} J_{m}(x)-x J_{m}^{\prime}(x)-x^{2} J_{m}^{\prime \prime}(x) .
$$

Therefore,

$$
I_{m, n}=\left.\frac{1}{2}\left[J_{m}(x)\right]^{2}\right|_{x=0} ^{x=\alpha_{m, n} a}-\int_{0}^{\alpha_{m, n} a} J_{m}^{\prime}(x)\left[m^{2} J_{m}(x)-x J_{m}^{\prime}(x)-x^{2} J_{m}^{\prime \prime}(x)\right] d x,
$$

or

$$
I_{m, n}=\left.\left\{\frac{x^{2}}{2}\left[J_{m}(x)\right]^{2}-\frac{m^{2}}{2}\left[J_{m}(x)\right]^{2}+\frac{x^{2}}{2}\left[J_{m}^{\prime}(x)\right]^{2}\right\}\right|_{x=0} ^{x=\alpha_{m, n} a} .
$$

Thus, if $J_{m}\left(\alpha_{m, n} a\right)=0$,

$$
N_{m, n}=\frac{a^{2}}{2}\left[J_{m}^{\prime}\left(\alpha_{m, n} a\right)\right]^{2}=\frac{a^{2}}{2}\left[J_{m+1}\left(\alpha_{m, n} a\right)\right]^{2}
$$

where we have made use of the recurrence relation

$$
\frac{d J_{m}}{d x}=-J_{m+1}(x)+\frac{m}{x} J_{m}(x)
$$

On the other hand, if $J_{m}^{\prime}\left(\alpha_{m, n} a\right)=0$,

$$
N_{m, n}=\frac{a^{2}}{2}\left(1-\frac{m^{2}}{\alpha_{m, n}^{2} a^{2}}\right)\left[J_{m}\left(\alpha_{m, n} a\right)\right]^{2} .
$$

Thus, combining the orthogonality and normalization results, we have either

$$
\int_{0}^{a} J_{m}\left(\alpha_{m, n} r\right) J_{m}\left(\alpha_{m, p} r\right) r d r=\frac{a^{2}}{2}\left[J_{m+1}\left(\alpha_{m, n} a\right)\right]^{2} \delta_{n, p}
$$

or

$$
\int_{0}^{a} J_{m}\left(\alpha_{m, n} r\right) J_{m}\left(\alpha_{m, p} r\right) r d r=\frac{a^{2}}{2}\left(1-\frac{m^{2}}{\alpha_{m, n}^{2} a^{2}}\right)\left[J_{m}\left(\alpha_{m, n} a\right)\right]^{2} \delta_{n, p} .
$$


The further consequence of being solutions of a Sturm-Liouville problem is the $J_{m}\left(\alpha_{m, n} r\right), n=1,2, \ldots$, are a basis for the space of functions that are squareintegrable with respect to the weight function $w(r)=r$ on the interval $0 \leq r \leq a$. Thus, any such function $f(r)$ can be represented by the (mean convergent) series

$$
f(r)=\sum_{n=1}^{\infty} c_{m, n} J_{m, n}\left(\alpha_{m, n} r\right)
$$

where

$$
c_{m, n}=\frac{2}{a^{2}\left[J_{m+1}\left(\alpha_{m, n} a\right)\right]^{2}} \int_{0}^{a} J_{m}\left(\alpha_{m, n} r\right) f(r) r d r,
$$

or

$$
c_{m, n}=\frac{2}{\left(a^{2}-\frac{m^{2}}{\alpha_{m, n}^{2}}\right)\left[J_{m}\left(\alpha_{m, n} a\right)\right]^{2}} \int_{0}^{a} J_{m}\left(\alpha_{m, n} r\right) f(r) r d r,
$$

depending on whether $\alpha_{m, n}=\frac{x_{m, n}}{a}$ or $\alpha_{m, n}=\frac{y_{m, n}}{a}, n=1,2, \ldots$

\subsubsection{Applications}

\section{Free Vibrations of a Circular Drum Head:}

The transverse vibrations of a (two-dimensional) drum head are described by the wave equation

$$
\nabla^{2} \psi=\frac{1}{c^{2}} \frac{\partial^{2} \psi}{\partial t^{2}} \text { where } c=\sqrt{\frac{\mathrm{T}}{\mu}}, \mathrm{T}=\text { tension/length, } \mu=\text { mass/area. }
$$

As we saw in Sections 10.4 and 10.8, the solution of this equation can be expressed as

$$
\psi(\boldsymbol{r}, t)=\sum_{n}\left[a_{n} \cos k_{n} c t+b_{n} \sin k_{n} c t\right] u_{n}(\boldsymbol{r})
$$

with

$$
\nabla^{2} u_{n}+k_{n}^{2} u_{n}=0 \text { and } u_{n}(\boldsymbol{r})=0 \text { for } \boldsymbol{r} \text { on the edge of the drum head, }
$$

where the latter condition follows from an assumption that the drum head is fixed along its edges. If it is a circular drum head, we should use cylindrical coordinates for $\boldsymbol{r}$. The solutions of the Helmholtz equation in these coordinates were obtained in Section 10.4. Since we have no z-dependence, the separation constant $\lambda_{2}$ in that discussion must be zero and so, $\alpha^{2}=k^{2}$. Thus, the solutions of the separated angular and radial equations are

$\Theta_{m}(\theta)=A_{m} \cos m \theta+B_{m} \sin m \theta$ and $R_{m}(r)=C_{m} J_{m}(k r)+D_{m} N_{m}(k r), \quad m=0,1,2, \ldots$ 
where we have imposed the boundary condition in $\theta$ but not those in $r$.

We shall take the radius of the drum head to be $a$ and its centre to be located at $r=0$. We then have as boundary conditions $|u(0, \theta)|<\infty$ and $u(a, \theta)=0$. The first of these implies that $D_{m}=0$ for all $m$. The second implies that $J_{m}(k a)=0$ which has solutions $k_{m, n}=\frac{x_{m, n}}{a}$ where $x_{m, n}$ is the nth zero of $J_{m}(x)$. This means that we obtain as eigenfunctions of the Helmholtz equation the normal modes

$$
u_{m, n}(r, \theta)=\left\{\begin{array}{l}
J_{m}\left(k_{m, n} r\right) \cos m \theta \\
J_{m}\left(k_{m, n} r\right) \sin m \theta
\end{array} \quad m=0,1,2, \ldots n=1,2, \ldots .\right.
$$

The frequencies $\omega_{m, n}=k_{m, n} c=x_{m, n} \frac{c}{a}, m=0,1,2, \ldots, n=1,2, \ldots$, are the natural frequencies of the drum head. Each frequency has two normal modes, one with $\cos m \theta$ and the other with $\sin m \theta$, and so is twofold degenerate. The exceptions are the frequencies with $m=0$ each of which has a single mode possessing radial symmetry. From the values of $x_{m, n}$ given above, we see that the lowest modes in order of frequency are

\begin{tabular}{ccc}
\hline$m, n$ & normal mode & frequency \\
$(0,1)$ & $J_{0}\left(k_{0,1} r\right)$ & $2.404 \frac{c}{a}$ \\
$(1,1)$ & $J_{1}\left(k_{1,1} r\right) \cos \theta, J_{1}\left(k_{1,1} r\right) \sin \theta$ & $3.832 \frac{c}{a}$ \\
$(2,1)$ & $J_{2}\left(k_{2,1} r\right) \cos 2 \theta, J_{2}\left(k_{2,1} r\right) \sin 2 \theta$ & $5.135 \frac{c}{a}$ \\
$(0,2)$ & $J_{0}\left(k_{0,2} r\right)$ & $5.520 \frac{c}{a}$ \\
$(3,1)$ & $J_{3}\left(k_{3,1} r\right) \cos 3 \theta, J_{3}\left(k_{3,1} r\right) \sin 3 \theta$ & $6.379 \frac{c}{a}$ \\
$(1,2)$ & $J_{1}\left(k_{1,2} r\right) \cos \theta, J_{1}\left(k_{1,2} r\right) \sin \theta$ & $7.016 \frac{c}{a}$ \\
$(4,1)$ & $J_{4}\left(k_{4,1} r\right) \cos 4 \theta, J_{4}\left(k_{4,1} r\right) \sin 4 \theta$ & $7.586 \frac{c}{a}$ \\
$(2,2)$ & $J_{2}\left(k_{2,2} r\right) \cos 2 \theta, J_{2}\left(k_{2,2} r\right) \sin 2 \theta$ & $8.417 \frac{c}{a}$ \\
$(0,3)$ & $J_{0}\left(k_{0,3} r\right)$ & $8.654 \frac{c}{a}$ \\
$(5,1)$ & $J_{5}\left(k_{5,1} r\right) \cos 5 \theta, J_{5}\left(k_{5,1} r\right) \sin 5 \theta$ & $8.779 \frac{c}{a}$ \\
\hline
\end{tabular}

To impose initial conditions on the transverse displacement and velocity of the drum head, we form the superposition of normal modes

$$
\begin{aligned}
\psi(r, \theta, t)= & \sum_{n=1}^{\infty} \sum_{m=0}^{\infty} J_{m}\left(k_{m, n} r\right)\left[\left(a_{m, n} \cos m \theta+b_{m, n} \sin m \theta\right) \cos \omega_{m, n} t\right. \\
& \left.+\left(c_{m, n} \cos m \theta+d_{m, n} \sin m \theta\right) \sin \omega_{m, n} t\right]
\end{aligned}
$$

At $t=0$ this gives us

$$
u_{0}(r, \theta) \equiv \psi(r, \theta, 0)=\sum_{n=1}^{\infty} \sum_{m=0}^{\infty} J_{m}\left(k_{m, n} r\right)\left[a_{m, n} \cos m \theta+b_{m, n} \sin m \theta\right]
$$

and

$$
\left.v_{0}(r, \theta) \equiv \frac{\partial \psi}{\partial t}\right|_{t=0}=\sum_{n=1}^{\infty} \sum_{m=0}^{\infty} J_{m}\left(k_{m, n} r\right) \omega_{m, n}\left[c_{m, n} \cos m \theta+d_{m, n} \sin m \theta\right]
$$


These are Fourier-Bessel series in $\mathrm{r}$ as well as Fourier series in $\theta$. Thus, invoking (11.3.29) for Fourier-Bessel coefficients and the Euler formulae for Fourier coefficients, we find

$$
\begin{gathered}
a_{m, n}=\frac{2}{\pi a^{2}\left[J_{m+1}\left(x_{m, n}\right)\right]^{2}}\left\{\begin{array}{l}
1 \\
\frac{1}{2}
\end{array}\right\} \int_{0}^{a} \int_{0}^{2 \pi} u_{0}(r, \theta) \cos m \theta J_{m}\left(k_{m, n} r\right) r d r d \theta \text { for }\left\{\begin{array}{l}
m \neq 0 \\
m=0
\end{array}\right\} \\
b_{m, n}=\frac{2}{\pi a^{2}\left[J_{m+1}\left(x_{m, n}\right)\right]^{2}} \int_{0}^{a} \int_{0}^{2 \pi} u_{0}(r, \theta) \sin m \theta J_{m}\left(k_{m, n} r\right) r d r d \theta \\
c_{m, n}=\frac{2}{\pi a^{2} \omega_{m, n}\left[J_{m+1}\left(x_{m, n}\right)\right]^{2}}\left\{\begin{array}{l}
1 \\
\frac{1}{2}
\end{array}\right\} \int_{0}^{a} \int_{0}^{2 \pi} v_{0}(r, \theta) \cos m \theta J_{m}\left(k_{m, n} r\right) r d r d \theta \text { for }\left\{\begin{array}{l}
m \neq 0 \\
m=0
\end{array}\right\} \\
d_{m, n}=\frac{2}{\pi a^{2} \omega_{m, n}\left[J_{m+1}\left(x_{m, n}\right)\right]^{2}} \int_{0}^{a} \int_{0}^{2 \pi} v_{0}(r, \theta) \sin m \theta J_{m}\left(k_{m, n} r\right) r d r d \theta .
\end{gathered}
$$

\section{Heat Conduction in a Cylinder of Finite Length:}

For our next application, we shall consider a metal cylinder of radius $R$ and length $L$ whose surface is maintained at a constant temperature $T_{1}$. Initially the cylinder is at a uniform temperature $T_{0}$. We want to find out how the temperature changes as a function of time and position.

We locate the cylinder so that its central axis lies along the z-axis and its ends correspond to $z=0$ and $z=L$. The temperature $\psi(\boldsymbol{r}, t)$ at any point within the cylinder and at any time $t$ will be a solution of the heat conduction equation

$$
\nabla^{2} \psi=\frac{1}{D} \frac{\partial \psi}{\partial t} \text { where } D=\frac{\kappa}{c \rho},
$$

$\kappa$ is the thermal conductivity, $c$ is the specific heat and $\rho$ is the density of the cylinder. Since $\psi(\boldsymbol{r}, t)=T_{1}=$ a constant is a solution of this equation, we can set $\psi(\boldsymbol{r}, t)=T_{1}+\psi_{1}(\boldsymbol{r}, t)$ where $\psi_{1}(\boldsymbol{r}, t)$ is a solution of the PDE that satisfies homogeneous boundary conditions at the surface of the cylinder. Separating variables, $\psi_{1}(\boldsymbol{r}, t)$ can be expressed as

$$
\psi_{1}(\boldsymbol{r}, t)=\sum_{\gamma} u_{\gamma}(\boldsymbol{r}) e^{-D k_{\gamma}^{2} t} \quad \text { where } \nabla^{2} u_{\gamma}+k_{\gamma}^{2} u_{\gamma}=0
$$

and $u_{\gamma}(\boldsymbol{r})$ is subject to homogeneous boundary conditions in all three coordinates. As ever, the requirement of single-valuedness implies that the angular dependence of the $u_{\gamma}$ is given by a linear combination of $\cos m \theta$ and $\sin m \theta$. Then, since $\left|u_{\gamma}(0, \theta, z)\right|<$ 
$\infty$ and $u_{\gamma}(R, \theta, z)=0$, the radial dependence of the $u_{\gamma}$ is determined by the Bessel functions $J_{m}\left(\alpha_{m, n} r\right), \alpha_{m, n}=\frac{x_{m, n}}{R}$. Thus, the $u_{\gamma}$ have the series representation

$$
\begin{aligned}
u_{\gamma}(r, \theta, z)= & \sum_{n=1}^{\infty} \sum_{m=0}^{\infty} J_{m}\left(\alpha_{m, n} r\right)\left[a_{m, n} \cos m \theta+b_{m, n} \sin m \theta\right] \\
& \times\left[c_{\gamma, m, n} e^{\sqrt{\alpha_{m, n}^{2}-k_{\gamma}^{2}} z}+d_{\gamma, m, n} e^{-\sqrt{\alpha_{m, n}^{2}-k_{\gamma}^{2}} z}\right] .
\end{aligned}
$$

Now we impose the requirement that $u_{\gamma}(r, \theta, 0)=u_{\gamma}(r, \theta, L)=0$. It then follows that

$$
c_{\gamma, m, n}=-d_{\gamma, m, n} \text { and } \sqrt{\alpha_{m, n}^{2}-k_{\gamma}^{2}}=i \beta, \quad \beta L=p \pi, \quad p=1,2, \ldots
$$

so that the final term in square brackets in $u_{\gamma}$ becomes proportional to $\sin \frac{p \pi z}{L}$. Thus,

$$
k_{\gamma}^{2}=k_{m, n, p}^{2}=\alpha_{m, n}^{2}+\beta^{2}=\frac{x_{m, n}^{2}}{R^{2}}+\frac{p^{2} \pi^{2}}{L^{2}}
$$

and the eigenfunctions (normal modes) corresponding to these eigenvalues are

$$
u_{m, n, p}(r, \theta, z)=J_{m}\left(\alpha_{m, n} r\right) \sin \frac{p \pi z}{L}\left[a_{m, n, p} \cos m \theta+b_{m, n, p} \sin m \theta\right] .
$$

To complete the solution we now must impose the initial condition on

$$
\begin{aligned}
& \psi(r, \theta, z, t)=T_{1}+ \\
& \sum_{p=1}^{\infty} \sum_{n=1}^{\infty} \sum_{m=0}^{\infty} J_{m}\left(\alpha_{m, n} r\right) \sin \frac{p \pi z}{L}\left[a_{m, n, p} \cos m \theta+b_{m, n, p} \sin m \theta\right] e^{-D k_{m, n, p}^{2} t} .
\end{aligned}
$$

Setting $t=0$, we have

$$
T_{0}-T_{1}=\sum_{p=1}^{\infty} \sum_{n=1}^{\infty} \sum_{m=0}^{\infty} J_{m}\left(\alpha_{m, n} r\right) \sin \frac{p \pi z}{L}\left[a_{m, n, p} \cos m \theta+b_{m, n, p} \sin m \theta\right] .
$$

Since the left hand side is constant, $m$ must be restricted to zero. Thus, we lose one summation and are left with the double series

$$
T_{0}-T_{1}=\sum_{p=1}^{\infty} \sum_{n=1}^{\infty} a_{0, n, p} J_{0}\left(\alpha_{0, n} r\right) \sin \frac{p \pi z}{L}
$$

which is a Fourier Bessel and Fourier sine series. Using the formulae for the coefficients of both such series, we find

$$
a_{0, n, p}=\frac{4\left(T_{0}-T_{1}\right)}{L R^{2}\left[J_{1}\left(x_{0, n}\right)\right]^{2}} \int_{0}^{R} \int_{0}^{L} J_{0}\left(\alpha_{0, n} r\right) \sin \frac{p \pi z}{L} d z r d r
$$

Since $x J_{0}(x)=\frac{d}{d x}\left[x J_{1}(x)\right]$, we have $\int_{0}^{a} J_{0}(x) x d x=a J_{1}(a)$. Therefore,

$$
\int_{0}^{R} J_{0}\left(\alpha_{0, n} r\right) r d r=\frac{1}{\alpha_{0, n}^{2}} \int_{0}^{\alpha_{0, n} R} J_{0}(x) x d x=\frac{R}{\alpha_{0, n}} J_{1}\left(\alpha_{0, n} R\right)=\frac{R^{2}}{\chi_{0, n}} J_{1}\left(x_{0, n}\right) .
$$


Further, the integral $\int_{0}^{L} \sin \frac{p \pi z}{L} d z=\frac{L}{p \pi}\left[1-(-1)^{p}\right]$ and so we obtain

$$
a_{0, n, p}=\frac{4\left(T_{0}-T_{1}\right)}{p \pi x_{0, n} J_{1}\left(x_{0, n}\right)}\left[1-(-1)^{p}\right]
$$

Our final solution for the temperature is thus

$$
\psi(r, \theta, z)=T_{1}+\frac{8\left(T_{0}-T_{1}\right)}{\pi} \sum_{p=1,3,5, \ldots}^{\infty} \sum_{n=1}^{\infty} \frac{1}{p x_{0, n} J_{1}\left(x_{0, n}\right)} J_{0}\left(\alpha_{0, n} r\right) \sin \frac{p \pi z}{L} e^{-D k_{0, n, p}^{2} t}
$$

where $k_{0, n}^{2}=\alpha_{0, n}^{2}+\frac{p^{2} \pi^{2}}{L^{2}}=\frac{x_{0, n}^{2}}{R^{2}}+\frac{p^{2} \pi^{2}}{L^{2}}$.

At the centre of the cylinder, $r=0$ and $z=\frac{L}{2}$. Therefore, since $J_{0}(0)=1$ and $\sin \frac{p \pi}{2}=(-1)^{\frac{p+1}{2}}$ for $p=$ an odd integer, we find a temperature

$$
T_{c} \equiv \psi\left(0, \theta, \frac{L}{2}\right)=T_{1}+\frac{8\left(T_{0}-T_{1}\right)}{\pi} \sum_{p=\text { odd }}^{\infty} \sum_{n=1}^{\infty} \frac{1}{p x_{0, n} J_{1}\left(x_{0, n}\right)}(-1)^{\frac{p-1}{2}} e^{-D k_{0, n, p}^{2} t} .
$$

For most metals, this series converges rapidly. For example, let us consider a steel cylinder of radius $R=0.1 \mathrm{~m}$, length $L=1 \mathrm{~m}$ and $D=0.126 \times 10^{-4} \frac{\mathrm{m}^{2}}{\mathrm{sec}}$. The tables of Bessel functions provide

$$
x_{0,1} \simeq 2.40, \quad x_{0,2} \simeq 5.52, \quad J_{1}(2.40) \simeq 0.52 \text { and } J_{1}(5.52) \simeq-0.34
$$

This means that successive values of $k_{0, n, p}^{2}$ are

$$
k_{0,1,1}^{2}=576, k_{0,1,3}^{2}=665 ., \quad k_{0,1,5}^{2}=823 ., \quad k_{0,2,1}^{2}=3.06 \times 10^{3}, \quad k_{0,1,3}^{2}=3.14 \times 10^{3}
$$

and, at time $t=3 \mathrm{mins}$, the corresponding exponents in successive terms of the series are

$$
-1.31,-1.51,-1.87,-6.93,-7.11 \text {. }
$$

This means in turn that $\exp \left[-\left(k_{0,2,1}^{2}-k_{0,1,1}^{2}\right) D t\right]=e^{-5.61} \times 0.004$ and so the $n=2$ terms can be ignored . Therefore, after 3 minutes, the central temperature will have decreased to

$$
\begin{aligned}
T_{c}= & T_{1}+\frac{8\left(T_{0}-T_{1}\right)}{\pi} \frac{1}{(2.40)(0.52)}\left[e^{-1.30}-\frac{1}{3} e^{-1.51}+\frac{1}{5} e^{-1.87}-\frac{1}{7} e^{-2.40}+-\ldots\right] \\
& \simeq T_{1}+\frac{8\left(T_{0}-T_{1}\right)}{\pi} \frac{(0.223)}{(2.40)(0.52)} \\
& \simeq T_{1}+(0.455)\left(T_{0}-T_{1}\right) .
\end{aligned}
$$

So, if the initial temperature is $500^{\circ} \mathrm{C}$ and the surface temperature is $20^{\circ} \mathrm{C}$, the centre will have cooled to $T_{c}=238^{\circ} \mathrm{C}$ in just 3 minutes. 


\section{Particle in a Cylindrical Box:}

The Schrödinger equation for a particle of mass $m$ confined to a box but otherwise not interacting with a field or with other particles is

$$
-\frac{\hbar^{2}}{2 m} \nabla^{2} \psi=E \psi
$$

with $\psi=0$ at the walls of the box. Setting $E=\frac{\hbar^{2} k^{2}}{2 m}$ and cancelling out common factors, we convert the PDE to Helmholtz' equation. Therefore, if the box is cylindrical, with radius $R$ and length $L$, we have precisely the same eigenvalue problem that we solved in the heat conduction problem. So, without further effort, we can assert that the energies available to the particle are $E_{m, n, p}=\frac{\hbar^{2}}{2 m}\left[\frac{x_{m, n}^{2}}{R^{2}}+\frac{p^{2} \pi^{2}}{L^{2}}\right]$ corresponding to the (unnormalized) wave functions

$$
\psi_{m, n, p}(r, \theta, z)=J_{m}\left(x_{m, n} \frac{r}{R}\right) \sin \frac{p \pi z}{L}\left\{\begin{array}{l}
\cos m \theta \\
\sin m \theta
\end{array}\right\}
$$

\section{Acoustic Radiation:}

As a final application, we shall consider sound waves in a gas contained within a cylindrical box or wave guide. One way of describing them is in terms of condensations and rarefactions or density fluctuations of the gas relative to a uniform background. The fluctuations, $\psi(\boldsymbol{r}, t) \equiv \frac{\rho(\boldsymbol{r}, t)-\rho_{0}}{\rho_{0}}$, where $\rho_{0}$ is the uniform background density, can be shown to satisfy the three-dimensional wave equation. Moreover, since there can be no motion normal to the (rigid) walls of the container, we know that the component of the gradient of the density that is normal to each wall must vanish at that wall. Thus, our problem is to solve

$$
\nabla^{2} \psi=\frac{1}{c^{2}} \frac{\partial^{2} \psi}{\partial t^{2}} \text { subject to }\left.\boldsymbol{n} \cdot \nabla \psi(\boldsymbol{r}, t)\right|_{\text {at the walls }}=0
$$

where $c$ is the speed of sound in the gas and is determined by $c^{2}=\frac{P_{0} \gamma}{\rho_{0}}, P_{0}$ is the background pressure and $\gamma$ is the ratio of heat capacities $\frac{C_{P}}{C_{V}}$.

Separating the time dependence, we again have

$$
\psi(r, t)=\sum_{\gamma} u_{\gamma}(r)\left[a_{\gamma} \cos k_{\gamma} c t+b_{\gamma} \sin k_{\gamma} c t\right]
$$

where the normal modes $u_{\gamma}(\mathrm{r})$ are determined by the eigenvalue problem

$$
\nabla^{2} u_{\gamma}+k_{\gamma}^{2} u_{\gamma}=0 \quad \text { with }\left.\boldsymbol{n} \cdot \nabla u_{\gamma}(\boldsymbol{r})\right|_{\text {at the walls }}=0
$$

Assuming a cylindrical container of radius $R$ and finite length $L$ and proceeding as in the preceding applications, our new boundary condition translates into the requirements

$$
\left.\frac{d}{d r} J_{m}(a r)\right|_{r=R}=0 \text { and }\left.\frac{d}{d z}\left[c_{\gamma} e^{\sqrt{a^{2}-k_{\gamma}^{2}}}+d_{\gamma} e^{\sqrt{a^{2}-k_{\gamma}^{2}}}\right]\right|_{z=0, L}=0
$$


rather than those requiring the undifferentiated functions to vanish. Therefore, in this case the eigenfunctions (normal modes) are

$$
u_{m n p}(r, \theta, z)=J_{m}\left(y_{m n} \frac{r}{R}\right) \cos \frac{p \pi z}{L}\left\{\begin{array}{c}
\cos m \theta \\
\sin m \theta
\end{array}\right\}
$$

with eigenvalues $k_{m n p}^{2}=\frac{y_{m n}^{2}}{R^{2}}+\frac{p^{2} \pi^{2}}{L^{2}}$ where $y_{m n}$ is the nth zero of $J_{m}^{\prime}(x)$. Initial values $\psi(\mathrm{r}, 0)$ and $\left.\frac{\partial \psi}{\partial t}\right|_{t=0}$ can now be fit to superpositions of these modes.

Of more interest is the propagation of acoustic waves along a very long cylindrical wave guide of radius $R$. Suppose that we generate the waves with a harmonic time dependence and frequency $\omega: \psi(r, \theta, z, t)=u(r, \theta, z) e^{-i \omega t}$. Substituting this into the wave equation, we obtain the Helmholtz equation $\nabla^{2} u+k^{2} u=0$ again but with $k^{2}$ already determined via $k^{2}=\frac{\omega^{2}}{c^{2}}$. Therefore, the solutions are

$$
u_{m n}(r, \theta, z)=J_{m}\left(\alpha_{m n} r\right)\left[a_{m n} \cos m \theta+b_{m n} \sin m \theta\right]\left[c_{m n} e^{\sqrt{\alpha_{m n}^{2}-k^{2}} z}+d_{m n} e^{-\sqrt{\alpha_{m n}^{2}-k^{2}} z}\right] .
$$

In order that these represent waves propagating down the wave guide in the positive z-direction, we require $d_{m n}=0$ and $\sqrt{\alpha_{m n}^{2}-\frac{\omega^{2}}{c^{2}}}=i \kappa_{m n}, \kappa_{m n}$ real, so that

$$
\psi_{m n}(r, \theta, z, t)=v_{m n}(r, \theta) e^{i\left(\kappa_{m n} z-\omega t\right)} \text { where } v_{m n}(r, \theta)=J_{m}\left(\frac{y_{m n}}{R} r\right)\left\{\begin{array}{l}
\cos m \theta \\
\sin m \theta
\end{array}\right\} .
$$

The modes that are allowed to propagate down the guide are those for which $\kappa_{m n}$ is indeed real. Since $\kappa_{m n}^{2}=\frac{\omega^{2}}{c^{2}}-\frac{y_{m n}^{2}}{R^{2}}$, we see that $\kappa_{m n}^{2}$ becomes negative for frequencies below the cut-off frequency $\omega_{m n}(\min )=\frac{c}{R} y_{m n}$ and the $(m, n)$ mode is not propagated. In fact, if one attempts to propagate the $(m, n)$ mode at a frequency $\omega<\omega_{m n}(\min )$, one will have $\kappa_{m n}^{2}=\frac{\omega^{2}-\omega_{m n}^{2}(\mathrm{~min})}{c^{2}}=-\beta_{m n}^{2}<0$ resulting in a wave number $\kappa_{m n}= \pm i \beta_{m n}$ that is pure imaginary and a wave

$$
\psi_{m n}(r, \theta, z, t)=v_{m n}(r, \theta) e^{-\beta_{m n} z-i \omega t}
$$

that is exponentially damped or attenuated. Notice that the $m=0, n=1$ mode is always propagated. This is because $y_{01}=0\left(J_{0}^{\prime}(x)=-J_{1}(x)\right)$ and since $J_{0}(0)=1$,

$$
\psi_{01}(r, \theta, z, t) \propto e^{i(k z-\omega t)}
$$

which is a plane wave propagating in the $+\mathrm{z}$-direction with wave number $\kappa_{01}=k=\frac{\omega}{c}$.

\subsubsection{Modified Bessel Functions}

The differential equation

$$
\frac{d^{2} y}{d x^{2}}+\frac{1}{x} \frac{d y}{d x}-\left(1+\frac{m^{2}}{x^{2}}\right) y=0
$$


is the same as Bessel's DE but with $x$ replaced by $i x$. Thus, its solutions are

$$
y(x)=\left\{\begin{array}{c}
J_{m}(i x) \\
N_{m}(i x)
\end{array}\right\}
$$

However, in physical applications it is convenient to have the solutions expressed in a form that is explicitly real for real values of $x$. Therefore, we define the modified Bessel functions

$$
I_{m}(x)=e^{-i m \frac{\pi}{2}} J_{m}(i x)=(-i)^{m} J_{m}(i x)=\sum_{k=0}^{\infty} \frac{1}{k !(k+m) !}\left(\frac{x}{2}\right)^{2 k},
$$

and

$$
K_{m}(x)=\frac{\pi}{2} i^{m+1} H_{m}^{(1)}(i x) .
$$

The choice of $K_{m}(x)$ as the second linearly independent solution is made to ensure that the two functions exhibit complementary asymptotic behaviour. Specifically, for $x \gg 1$,

$$
I_{m}(x) \sim \sqrt{\frac{1}{2 \pi x}} e^{x} \text { and } K_{m}(x) \sim \sqrt{\frac{\pi}{2 x}} e^{-x} .
$$

For small values of $x, x \ll 1$, the modified Bessel functions have the limiting forms

$$
\begin{aligned}
& I_{m}(x) \simeq\left\{\begin{array}{cc}
\frac{1}{m !}\left(\frac{x}{2}\right)^{m} & \text { for } m>0 \\
1 & \text { for } m=0
\end{array},\right. \text { and } \\
& K_{m}(x) \simeq\left\{\begin{array}{ll}
\frac{(m-1) !}{2}\left(\frac{x}{2}\right)^{-m} & \text { for } m>0 \\
-\left(\ln \frac{x}{2}+\gamma\right) & \text { for } m=0
\end{array} .\right.
\end{aligned}
$$

Note that the $I_{m}(x)$ is well behaved at the origin but diverges at infinity while the reverse is true for $K_{m}(x)$. Like the hyperbolic functions, neither $I_{m}(x)$ nor $K_{m}(x)$ has multiple zeros.

Not surprisingly, the recurrence relations satisfied by the modified Bessel functions are similar to those satisfied by $J_{m}(x)$. The most important ones are

$$
\begin{gathered}
\operatorname{Im}_{m+1}(x)+I_{m-1}(x)=2 I_{m}^{\prime}(x), \\
\frac{d}{d x}\left[x^{m} I_{m}(x)\right]=x^{m} I_{m-1}(x) \text { and } \frac{d}{d x}\left[\frac{I_{m}(x)}{x^{m}}\right]=\frac{I_{m+1}(x)}{x^{m}} \\
I_{m-1}(x)-I_{m+1}(x)=\frac{2 m}{x} I_{m}(x),
\end{gathered}
$$


for $I_{m}(x)$ and

$$
\begin{gathered}
K_{m+1}(x)+K_{m-1}(x)=-2 K_{m}^{\prime}(x) \\
\frac{d}{d x}\left[x^{m} K_{m}(x)\right]=-x^{m} K_{m-1}(x) \text { and } \frac{d}{d x}\left[\frac{K_{m}(x)}{x^{m}}\right]=-\frac{K_{m+1}(x)}{x^{m}} \\
K_{m-1}(x)-K_{m+1}(x)=-\frac{2 m}{x} K_{m}(x)
\end{gathered}
$$

for $K_{m}(x)$.

Because the modified Bessel functions do not have multiple zeros, they cannot satisfy homogeneous boundary conditions of the type found in Sturm-Liouville problems and so do not comprise complete orthogonal sets. Thus, when they figure in the solution of a boundary value problem, they are always coupled with functions that do form complete orthogonal sets. As we shall see in the next sub-section, potential problems with cylindrical symmetry provide a graphic illustration of this point.

\subsubsection{Electrostatic Potential in and around Cylinders}

Consider a cylinder of radius $R$ and height $L$. One surface, either the top or the curved lateral wall of the cylinder, is maintained at a non-zero and perhaps variable electrostatic potential $V$. We want to find the potential $\psi(r, \theta, z)$ at any point inside the cylinder. Since there are no charges present, the potential must satisfy Laplace's equation, $\nabla^{2} \psi=0$. Using cylindrical coordinates and then separating variables in Section 8.4, we found that the solutions of this equation could be expressed as one of two possible superpositions: either

$$
\psi(r, \theta, z)=\sum_{\alpha, m}\left\{\begin{array}{c}
J_{m}(\alpha r) \\
N_{m}(\alpha r)
\end{array}\right\}\left\{\begin{array}{c}
\cosh \alpha z \\
\sinh \alpha z
\end{array}\right\}\left\{\begin{array}{c}
\cos m \theta \\
\sin m \theta
\end{array}\right\}, \alpha^{2}>0
$$

or

$$
\psi(r, \theta, z)=\sum_{\alpha, m}\left\{\begin{array}{c}
I_{m}(|\alpha| r) \\
K_{m}(|\alpha| r)
\end{array}\right\}\left\{\begin{array}{c}
\cos |\alpha| z \\
\sin |\alpha| z
\end{array}\right\}\left\{\begin{array}{c}
\cos m \theta \\
\sin m \theta
\end{array}\right\}, \alpha^{2}<0
$$

where, as usual, each set of braces is understood to be a linear combination of the functions they contain. As we noted then, both superpositions have the $r$ and $z$ dependence coupled in such a way that one or the other but not both is oscillatory in behaviour. Therefore, the choice between the two options is made for us by the boundary conditions in the problem: to satisfy homogeneous conditions at both boundaries associated with a particular variable, we require a function with multiple zeros and thus an oscillatory dependence on that variable. 


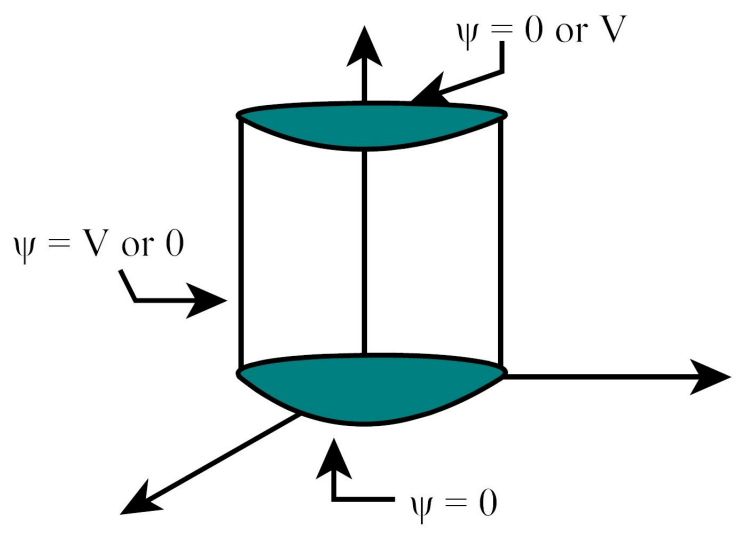

So, let us start with the boundary conditions

$$
\psi(r, \theta, 0)=\psi(r, \theta, L)=0 \text { and } \psi(R, \theta, z)=V(\theta, z) .
$$

To satisfy the homogeneous boundary conditions at the $z$ boundaries, we must choose the second superposition, discard the $\cos |\alpha| z$ possibility and set $\alpha^{2}=-\frac{n^{2} \pi^{2}}{L^{2}}, n=$ $1,2, \ldots$ Next, the (implied) boundary condition $|\psi(0, \theta, z)|<\infty$ requires that we discard the $K_{m}(|\alpha| r)$ possibility and so we arrive rather quickly at the following expression for the potential inside the "can":

$$
\psi(r, \theta, z)=\sum_{m=0}^{\infty} \sum_{n=1}^{\infty} I_{m}\left(\frac{n \pi r}{L}\right) \sin \frac{n \pi z}{L}\left[a_{m n} \cos m \theta+b_{m n} \sin m \theta\right] .
$$

This double Fourier series can now be made to fit the remaining boundary condition $\psi(R, \theta, z)=V(\theta, z)$ and thus determine the coefficients $a_{m n}, b_{m n}$ :

$$
\begin{gathered}
a_{m n}=\frac{2}{\pi L I m\left(\frac{n \pi R}{L}\right)}\left\{\begin{array}{c}
1 \\
\frac{1}{2}
\end{array}\right\} \int_{0}^{L} \int_{0}^{2 \pi} V(\theta, z) \sin \frac{n \pi z}{L} \cos m \theta d \theta d z \text { for }\left\{\begin{array}{c}
m \neq 0 \\
m=0
\end{array}\right\}, \\
b_{m n}=\frac{2}{\pi L I_{m}\left(\frac{n \pi R}{L}\right)} \int_{0}^{L} \int_{0}^{2 \pi} V(\theta, z) \sin \frac{n \pi z}{L} \sin m \theta d \theta d z .
\end{gathered}
$$

Suppose, for example, that we halve the cylinder vertically and insert the usual very thin strip of perfect insulator to permit a potential $V(\theta, z)=\left\{\begin{aligned} V_{0} & \text { for } 0<\theta<\pi \\ -V_{0} & \text { for } \pi<\theta<2 \pi\end{aligned}\right.$ where $V_{0}$ is a constant. Since it is an odd function of $\theta, a_{m n}=0$ for all $m$ and

$$
b_{m n}=\frac{4 V_{0}}{\pi L I_{m}\left(\frac{n \pi R}{L}\right)} \int_{0}^{L} \int_{0}^{\pi} \sin \frac{n \pi z}{L} \sin m \theta d \theta d z .
$$


Since $\int_{0}^{L} \sin \frac{n \pi z}{L} d z=\frac{L}{n \pi}\left[1-(-1)^{n}\right]$ and $\int_{0}^{\pi} \sin m \theta d \theta=\frac{1}{m}\left[1-(-1)^{m}\right]$, we have

$$
b_{m, n}=\left\{\begin{array}{cc}
\frac{1}{m n} \frac{16 V_{0}}{\pi^{2} I_{m}\left(\frac{n \pi R}{L}\right)} & \text { for } m \text { and } n \text { odd } \\
0 & \text { for } m \text { and } n \text { even }
\end{array} .\right.
$$

Thus,

$$
\psi(r, \theta, z)=\frac{16 V_{0}}{\pi^{2}} \sum_{m=1,3, \ldots}^{\infty} \sum_{n=1,3, \ldots}^{\infty} \frac{1}{m n} \frac{I_{m}\left(\frac{n \pi r}{L}\right)}{I_{m}\left(\frac{n \pi R}{L}\right)} \sin m \theta \sin \frac{n \pi z}{L} .
$$

If, on the other hand, we had started with the boundary conditions $\psi(R, \theta, z)=0$, $\psi(r, \theta, 0)=0$ and $\psi(r, \theta, L)=V(r, \theta)$, we would have been obliged to go with the first superposition, discarded the $N_{m}(\alpha r)$ possibility (to ensure boundedness at the origin) and the $\cosh \alpha z$ possibility (to meet the condition at $z=0$ ), and set $\alpha=\alpha_{m n}=\frac{x_{m n}}{R}$ where $x_{m n}$ is the nth zero of $J_{m}(x)$. Thus, our expression for the potential inside the "can" would become

$$
\psi(r, \theta, z)=\sum_{m=0}^{\infty} \sum_{n=1}^{\infty} J_{m}\left(\alpha_{m n} r\right) \sinh \alpha_{m n} z\left[a_{m n} \cos m \theta+b_{m n} \sin m \theta\right]
$$

where

$a_{m n}=\frac{2 \operatorname{cosech} \alpha_{m n} L}{\pi R^{2}\left[J_{m+1}\left(\alpha_{m n} R\right)\right]^{2}}\left\{\begin{array}{c}1 \\ \frac{1}{2}\end{array}\right\} \int_{0}^{2 \pi} \int_{0}^{R} V(r, \theta) J_{m}\left(\alpha_{m n} r\right) \cos m \theta r d r d \theta$ for $\left\{\begin{array}{l}m \neq 0 \\ m=0\end{array}\right\}$

and

$$
b_{m n}=\frac{2 \operatorname{cosech} \alpha_{m n} L}{\pi R^{2}\left[J_{m+1}\left(\alpha_{m n} R\right)\right]^{2}} \int_{0}^{2 \pi} \int_{0}^{R} V(r, \theta) J_{m}\left(\alpha_{m n} r\right) \sin m \theta r d r d \theta .
$$

As an example, let us take $V(r, \theta)=V_{0}=$ a constant. We would then have $b_{m n}=0$ for all $m$ and $a_{m n}=0$ for all $m \neq 0$. Thus,

$$
\psi(r, \theta, z)=\sum_{n=1}^{\infty} a_{0, n} J_{0}\left(\alpha_{0, n} r\right) \sinh \alpha_{0, n} z
$$

with

$$
a_{0 n}=\frac{2 V_{0} \operatorname{cosech} \alpha_{0 n} L}{R^{2}\left[J_{1}\left(\alpha_{0 n} R\right)\right]^{2}} \int_{0}^{R} J_{0}\left(\alpha_{0 n} r\right) r d r
$$

As we learned in sub-section 11.3.2, $\int_{0}^{R} J_{0}\left(\alpha_{0 n} r\right) r d r=\frac{R}{\alpha_{0 n}} J_{1}\left(\alpha_{0 n} R\right)$. Therefore, our final expression for the potential under these boundary conditions is

$$
\psi(r, \theta, z)=2 V_{0} \sum_{n=1}^{\infty} \frac{J_{0}\left(x_{0 n} \frac{r}{R}\right)}{x_{0 n} J_{1}\left(x_{0 n}\right)} \frac{\sinh \left(x_{0 n} \frac{z}{R}\right)}{\sinh \left(x_{0 n} \frac{L}{R}\right)} .
$$


As a final example, we shall allow our cylinder to extend infinitely far in both the positive and negative $z$ directions. The boundary conditions at the $z$ boundaries must become $|\psi(r, \theta, z)|<\infty$ which immediately implies that $\alpha=i k,-\infty<k<\infty$. The appropriate superposition to represent $\psi$ must then be

$$
\psi(r, \theta, z)=\sum_{m=0}^{\infty}\left\{\int_{-\infty}^{\infty}\left[A_{m}(k) I_{m}(|k| r)+B_{m}(k) K_{m}(|k| r)\right] e^{i k z} d k\right\}\left\{\begin{array}{c}
\cos m \theta \\
\sin m \theta
\end{array}\right\}
$$

where the summation over $\alpha$ has become a continuous sum or integral over $k$.

Suppose that we are interested in finding the potential outside the cylinder, given that it is maintained at a value $V(\theta, z)$ on the curved surface. The boundary conditions in $r$ must then be $\psi(R, \theta, z)=V(\theta, z)$ plus the requirement that the potential be bounded as $r \rightarrow \infty,|\psi(\infty, \theta, z)|<\infty$. But we know from the asymptotic behaviour of the modified Bessel functions given in (11.3.34) that this latter requirement eliminates the $I_{m}^{\prime} s$ from consideration. Thus,

$$
\psi(r, \theta, z)=\sum_{m=0}^{\infty} \int_{-\infty}^{\infty} K_{m}(|k| r) e^{i k z}\left[a_{m}(k) \cos m \theta+b_{m}(k) \sin m \theta\right] d k
$$

where

$$
a_{m}(k)=\frac{1}{2 \pi^{2} K_{m}(|k| R)}\left\{\begin{array}{c}
1 \\
\frac{1}{2}
\end{array}\right\} \int_{0}^{2 \pi} \int_{-\infty}^{\infty} V(\theta, z) e^{-i k z} \cos m \theta d \theta d z \text { for }\left\{\begin{array}{l}
m \neq 0 \\
m=0
\end{array}\right\}
$$

and,

$$
b_{m}(k)=\frac{1}{2 \pi^{2} K_{m}(|k| R)} \int_{0}^{2 \pi} \int_{-\infty}^{\infty} V(\theta, z) e^{-i k z} \sin m \theta d \theta d z
$$

Taking the relatively simple functional form $V(\theta, z)=V_{0} e^{-c|z|}, V_{0}$ and $c$ constants, results in a restriction to $m=0$ and yields

$$
a_{0}(k)=\frac{V_{0}}{2 \pi K_{m}(|k| R)} \int_{-\infty}^{\infty} e^{-c|z|} e^{-i k z} d z=\frac{V_{0}}{\pi K_{m}(|k| R)} \frac{c}{c^{2}+k^{2}} .
$$

Thus, in this case, our solution for the potential outside the cylinder becomes

$$
\psi(r, \theta, z)=\frac{V_{0}}{\pi} \int_{-\infty}^{\infty} \frac{K_{m}(|k| r)}{K_{m}(|k| R)} \frac{c}{c^{2}+k^{2}} e^{i k z} d k
$$

\subsubsection{Fourier-Bessel Transforms}

As we have seen repeatedly, including the preceding example, when homogeneous boundary conditions are imposed at the limits of an infinite or semi-infinite range the 
eigenvalue spectrum that results is continuous rather than discrete and the superposition of eigensolutions requires an integration or, more precisely, an integral transformation. A further example is provided by Laplace's equation in cylindrical coordinates with the homogeneous boundary conditions that $\psi$ be bounded both at $r=0$ and as $r \rightarrow \infty$. Bessel's DE becomes a Sturm-Liouville eigenvalue equation again but this time the eigenfunction solutions are the set $J_{m}(\alpha r), 0<\alpha<\infty$. To arrive at this conclusion, we simply have to note that neither of the modified Bessel functions nor the Neumann function can be bounded at both limits while the Bessel function of the first kind obviously can.

To conform with more conventional notation we shall set $\alpha=k, 0<k<\infty$. We then have, from the properties of Sturm-Liouville eigenfunctions, orthogonality and completeness with respect to the weight function $\rho(r)=r$. As it happens, the Bessel functions are appropriately normalized already for this range of $r$ and so the statement of orthogonality is

$$
\int_{0}^{\infty} J_{m}(k r) J_{m}\left(k^{\prime} r\right) r d r=\frac{1}{k} \delta\left(k-k^{\prime}\right)
$$

while the closure relation is

$$
\int_{0}^{\infty} J_{m}(k r) J_{m}\left(k r^{\prime}\right) k d k=\frac{1}{r} \delta\left(r-r^{\prime}\right) .
$$

The proof of the orthogonality statement follows from an application of equation (10.5.25) and the generalized Green's identity (10.5.18) to these eigenfunctions of the Bessel differential operator. The result is

$$
\left.r\left[J_{m}(k r) \frac{\partial J_{m}\left(k^{\prime} r\right)}{\partial r}-J_{m}\left(k^{\prime} r\right) \frac{\partial J_{m}(k r)}{\partial r}\right]\right|_{0} ^{\infty}=\left[k^{2}-\left(k^{\prime}\right)^{2}\right] \int_{0}^{\infty} J_{m}(k r) J_{m}\left(k^{\prime} r\right) r d r .
$$

If we now use the recurrence relation (11.3.16) and the asymptotic form (11.3.6), we can express the left hand side of this equation as

$$
\lim _{r \rightarrow \infty} \frac{1}{\pi}\left[\frac{k+k^{\prime}}{\sqrt{k k^{\prime}}} \sin \left(k-k^{\prime}\right) r-\frac{k-k^{\prime}}{\sqrt{k k^{\prime}}}(-1)^{m} \cos \left(k+k^{\prime}\right) r\right] .
$$

Thus, dividing through by $\left[k^{2}-\left(k^{\prime}\right)^{2}\right]$, we have

$$
\int_{0}^{\infty} J_{m}(k r) J_{m}\left(k^{\prime} r\right) r d r=\lim _{r \rightarrow \infty} \frac{1}{\pi}\left[\frac{1}{\sqrt{k k^{\prime}}} \frac{\sin \left(k-k^{\prime}\right) r}{k-k^{\prime}}+\frac{(-1)^{m}}{\sqrt{k k^{\prime}}} \frac{\cos \left(k+k^{\prime}\right) r}{k+k^{\prime}}\right] .
$$

The first term on the right hand side, in that limit, is a representation of $\frac{1}{\sqrt{k k^{\prime}}} \delta\left(k-k^{\prime}\right)=$ $\frac{1}{k} \delta\left(k-k^{\prime}\right)$ while the second has a limit of zero. Thus, we get the result in equation (11.3.43). 
The statement of completeness is that any function $f(r)$ that is square-integrable with respect to the weight function $\rho(r)=r$ can be represented by the (mean) convergent integral

$$
f(r)=\int_{0}^{\infty} F(k) J_{m}(k r) k d k
$$

where

$$
F(k)=\int_{0}^{\infty} f(r) J_{m}(k r) r d r .
$$

The functions $f(r)$ and $F(k)$ are Fourier-Bessel Transforms of each other.

An application for these transforms is the solution of Laplace's equation in the space between two infinite planes located at $z=0$ and $z=L$ with $\psi$ specified on the planes themselves and with the assumption that $|\psi| \rightarrow 0$ as $x$ and $y$ or $r \rightarrow \infty$. In a problem like this we have the option of using either Cartesian or cylindrical coordinates. If we choose the former, $\psi$ will be represented by a double Fourier integral transform involving the $x$ and $y$ variables. If the latter, we will have a Fourier-Bessel transform for the $r$-dependence and a Fourier series for the $\theta$-dependence. We shall illustrate with a specific example.

The electrostatic potential on a plane at $z=0$ is given by the function

$$
V(r, \theta)=\left\{\begin{array}{cc}
0 & \text { for } r<1 \\
\frac{V_{0}}{\sqrt{r^{2}-1}} & \text { for } r>1
\end{array} .\right.
$$

We seek the potential everywhere above the plane given that it goes to zero uniformly as $z \rightarrow \infty$ and as $r \rightarrow \infty$. Evidently, cylindrical coordinates are appropriate and so we represent the solution with the superposition

$$
\psi(r, \theta, z)=\sum_{m=0}^{\infty}\left\{\begin{array}{c}
\cos m \theta \\
\sin m \theta
\end{array}\right\} \int_{0}^{\infty} J_{m}(k r)\left\{\begin{array}{c}
e^{k z} \\
e^{-k z}
\end{array}\right\} k d k .
$$

The boundary conditions in this problem provide considerable simplification: because $|\psi| \rightarrow 0$ as $z \rightarrow \infty$ we discard the $e^{k z}$ possibility and because $\psi$ is independent of $\theta$ when $z=0$ we restrict $m$ to be zero. Thus,

$$
\psi(r, \theta, z)=\int_{0}^{\infty} A_{0}(k) e^{-k z} J_{0}(k r) k d k
$$

Therefore, since $\psi(r, \theta, 0)=V(r, \theta)=\int_{0}^{\infty} A_{0}(k) J_{0}(k r) k d k$, we have

$$
A_{0}(k)=\int_{1}^{\infty} \frac{V_{0}}{\sqrt{r^{2}-1}} J_{0}(k r) r d r=\frac{V_{0}}{k} \cos k
$$


where we have used integral 6.554\#3 from Gradshteyn and Ryzhik. Substituting this back into (11.3.48), we obtain the solution

$$
\psi(r, \theta, z)=V_{0} \int_{0}^{\infty} \cos k e^{-k z} J_{0}(k r) d k .
$$

Using Gradshteyn and Ryzhik one more time, formula 6.611\#1 in this case, we can evaluate this explicitly and find

$$
\psi(r, \theta, z)=V_{0} \operatorname{Re}\left[\frac{1}{\sqrt{\left(z^{2}+r^{2}-1\right)+2 i z}}\right], \quad z \geq 0, \quad r>1 .
$$

\subsection{Spherical Bessel Functions: Spherical Waves}

\subsubsection{Properties of Spherical Bessel Functions}

In Section 10.4 we discovered that separation of variables applied to the Helmholtz equation $\nabla^{2} u(\boldsymbol{r})+k^{2} u(\boldsymbol{r})=0$ when spherical coordinates are used results in a radial equation

$$
\frac{d^{2} R}{d r^{2}}+\frac{2}{r} \frac{d R}{d r}+\left[k^{2}-\frac{l(l+1)}{r^{2}}\right] R=0
$$

with general solution

$$
R(r)=\left\{\begin{array}{l}
j_{l}(k r) \\
n_{l}(k r)
\end{array}\right\}
$$

The functions $j_{l}(k r)$ and $n_{l}(k r)$ are called spherical Bessel and Neumann functions and are defined by

$$
j_{l}(x)=\sqrt{\frac{\pi}{2 x}} J_{l+\frac{1}{2}}(x) \text { and } n_{l}(x)=\sqrt{\frac{\pi}{2 x}} N_{l+\frac{1}{2}}(x)=(-1)^{l+1} \sqrt{\frac{\pi}{2 x}} J_{-l-\frac{1}{2}}(x) .
$$

From the power series representation of $J_{m}(x)$ we have

$$
J_{l+\frac{1}{2}}(x)=\sum_{k=0}^{\infty} \frac{(-1)^{k}}{k ! \Gamma\left(k+l+\frac{3}{2}\right)}\left(\frac{x}{2}\right)^{2 k+l+\frac{1}{2}} .
$$

Thus, using

$$
\Gamma\left(\frac{1}{2}+n+1\right)=\frac{(2 n+1)(2 n-1) \ldots 1}{2^{n+1}} \Gamma\left(\frac{1}{2}\right)=\frac{(2 n+1) !}{2^{2 n+1} n !} \sqrt{\pi},
$$

we find

$$
j_{l}(x)=2^{l} \sum_{k=0}^{\infty} \frac{(-1)^{k}(k+l) !}{k !(2 k+2 l+1) !} x^{2 k+l} \text {. }
$$


Similarly, starting from the power series representation of $J_{-l-\frac{1}{2}}(x)$, one finds

$$
n_{l}(x)=\frac{(-1)^{l}}{2^{l}} \sum_{k=0}^{\infty} \frac{(-1)^{k} \Gamma(k-l+1)}{k ! \Gamma(2 k-2 l+1)} x^{2 k-l-1} .
$$

These two power series are instantly recognizable in the special case of $l=0$ :

$$
j_{0}(x)=\sum_{k=0}^{\infty} \frac{(-1)^{k}}{(2 k+1) !} x^{2 k}=\frac{\sin x}{x}
$$

and

$$
n_{0}(x)=(-1) \sum_{k=0}^{\infty} \frac{(-1)^{k}}{(2 k) !} x^{2 k-1}=-\frac{\cos x}{x}
$$

where we have used $\Gamma(2 n+1)=(2 n)$ ! and $\Gamma(n+1)=n$ !. These two identities offer a valuable aid to one's intuitive appreciation of $j_{0}(x)$ and $n_{0}(x)$.

From the recurrence relations satisfied by $J_{l+\frac{1}{2}}(x)$ and $J_{-l-\frac{1}{2}}(x)$, one can derive corresponding relations for $j_{l}(x)$ and $n_{l}(x)$. One finds that they both satisfy

$$
j_{l-1}(x)+j_{l+1}(x)=\frac{2 l+1}{x} j_{l}(x),
$$

and

$$
l j_{l-1}(x)-(l+1) j_{l+1}(x)=(2 l+1) \frac{d j_{l}(x)}{d x} .
$$

Multiplying the first of these by $l$ and then subtracting the second from it, we find

$$
j_{l+1}(x)=-x^{l} \frac{d}{d x}\left(\frac{j_{l}(x)}{x^{l}}\right) .
$$

We can generate successive $j_{l}^{\prime} s$ and $n_{l}^{\prime} s$ by applying this relation repeatedly to $j_{0}(x)$ and $n_{0}(x)$, respectively. Thus, we write formally

$$
j_{l}(x)=x^{l}\left(-\frac{1}{x} \frac{d}{d x}\right)^{l} j_{0}(x)=(-1)^{l} x^{l}\left(\frac{1}{x} \frac{d}{d x}\right)^{l}\left(\frac{\sin x}{x}\right), \quad l=1,2, \ldots
$$

and

$$
n_{l}(x)=x^{l}\left(-\frac{1}{x} \frac{d}{d x}\right)^{l} n_{0}(x)=(-1)^{l+1} x^{l}\left(\frac{1}{x} \frac{d}{d x}\right)^{l}\left(\frac{\cos x}{x}\right), l=1,2, \ldots
$$

Evidently all spherical Bessel and Neumann functions can be expressed in terms of sines and cosines. For example,

$$
j_{1}(x)=\frac{\sin x}{x^{2}}-\frac{\cos x}{x}, \quad n_{1}(x)=-\frac{\cos x}{x^{2}}-\frac{\sin x}{x}
$$




$$
j_{2}(x)=\left(\frac{3}{x^{3}}-\frac{1}{x}\right) \sin x-\frac{3}{x^{2}} \cos x, n_{2}(x)=-\left(\frac{3}{x^{3}}-\frac{1}{x}\right) \cos x-\frac{3}{x^{2}} \sin x .
$$

The spherical Hankel functions are defined by analogy with ordinary Hankel functions:

$$
\begin{aligned}
& h_{l}^{(1)}(x)=j_{l}(x)+i n_{l}(x)=(-x)^{l}\left(\frac{1}{x} \frac{d}{d x}\right)^{l}\left(\frac{e^{i x}}{i x}\right), \\
& h_{l}^{(2)}(x)=j_{l}(x)-i n_{l}(x)=(-x)^{l}\left(\frac{1}{x} \frac{d}{d x}\right)^{l}\left(\frac{e^{-i x}}{-i x}\right) .
\end{aligned}
$$

The small $x$ behaviour of these functions is easily obtained from their power series representations:

$$
\begin{gathered}
j_{l}(x) \simeq \frac{2^{l} l !}{(2 l+1) !} x^{l}=\frac{x^{l}}{(2 l+1) ! !}=\frac{x^{l}}{(2 l+1) \ldots 5 \cdot 3 \cdot 1}, \text { for } x \ll 1, \\
n_{l}(x) \simeq-\frac{(2 l) !}{2^{l} l !} \frac{1}{x^{l+1}}, \quad \text { for } x \ll 1 .
\end{gathered}
$$

To obtain the asymptotic behaviour, we could start from the behaviour reported for the Bessel and Neumann functions in Section 11.3.1. A more direct approach however is to use equations (11.4.13) and (11.4.14) for the Hankel functions. For very large $x, x \gg 1$ or $l$, the largest contributions in these formulas results from applying all derivatives to $e^{i x}$ rather than the inverse powers of $x$. Thus, we find

$$
\begin{gathered}
h_{l}^{(1)}(x) \sim(-1)^{l}(i)^{l} \frac{e^{i x}}{i x}=\frac{1}{x} e^{i\left[x-(l+1) \frac{\pi}{2}\right]} \text { for } x \gg 1, l, \\
h_{l}^{(2)}(x) \sim \frac{1}{x} e^{-i\left[x-(l+1) \frac{\pi}{2}\right]} \text { for } x \gg 1, l .
\end{gathered}
$$

Combining these to construct $j_{l}(x)$ and $n_{l}(x)$ we obtain

$$
\begin{aligned}
& j_{l}(x) \sim \frac{1}{x} \cos \left(x-(l+1) \frac{\pi}{2}\right)=\frac{1}{x} \sin \left(x-l \frac{\pi}{2}\right) \text { for } x \gg 1, l, \\
& n_{l}(x) \sim \frac{1}{x} \sin \left(x-(l+1) \frac{\pi}{2}\right)=-\frac{1}{x} \cos \left(x-l \frac{\pi}{2}\right) \text { for } x \gg 1, l .
\end{aligned}
$$

The spherical Bessel and Neumann functions $j_{l}(k r)$ and $n_{l}(k r)$ are solutions of

$$
\frac{d}{d r}\left(r^{2} \frac{d R_{l}}{d r}\right)-l(l+1) R_{l}(r)=-k^{2} r^{2} R_{l}(r)
$$

which is of the Sturm-Liouville form. If we impose homogeneous boundary conditions such as

$$
\left|R_{l}(0)\right|<\infty \text { and } R_{l}(a)=0 \text {, }
$$


it becomes an eigenvalue equation with eigenvalue $\lambda=k^{2}$ and weight function $\rho(r)=$ $r^{2}$. The first boundary condition requires us to discard the possibility of $n_{l}(k r)$ figuring in the solution. The second condition requires $j_{l}(k a)=0$ or

$$
k a=k_{l, n} a=z_{l, n} \equiv\left\{\text { the nth zero of } j_{l}(x)\right\}=x_{l+\frac{1}{2}, n} \equiv\left\{\text { the nth zero of } J_{l+\frac{1}{2}}(x)\right\} \text {. }
$$

Thus, the eigenfunctions that correspond to these boundary conditions are $j_{l}\left(k_{l, n} r\right)$.

Eigenfunctions associated with different eigenvalues are orthogonal with respect to the weight function $\rho(r)=r^{2}$ :

$$
\int_{0}^{a} j_{l}\left(k_{l, n} r\right) j_{l}\left(k_{l, m} r\right) r^{2} d r=0 \text { for } n \neq m .
$$

The normalization of the eigenfunctions follows from that for ordinary Bessel functions:

$$
\begin{aligned}
\int_{0}^{a}\left[j_{l}\left(k_{l, n} r\right)\right]^{2} r^{2} d r & =\frac{\pi}{2 k_{l, n}} \int_{0}^{a}\left[J_{l+\frac{1}{2}}\left(k_{l, n} r\right)\right]^{2} r d r=\frac{\pi}{2 k_{l, n}} \frac{a^{2}}{2}\left[J_{l+\frac{1}{2}}^{\prime}\left(k_{l, n} a\right)\right]^{2} \\
& =\frac{a^{3}}{2}\left[j_{l}^{\prime}\left(k_{l, n} a\right)\right]^{2} .
\end{aligned}
$$

If we now use the recurrence relation $j_{l}^{\prime}(x)=\frac{l}{x} j_{l}(x)-j_{l+1}(x)$, we can give this result the alternative expression

$$
\int_{0}^{a}\left[j_{l}\left(k_{l, n} r\right)\right]^{2} r^{2} d r=\frac{a^{3}}{2}\left[j_{l+1}\left(k_{l, n} a\right)\right]^{2} .
$$

The eigenfunctions form a complete as well as an orthogonal set. Thus, any function that is square integrable with respect to $r^{2}$ on the interval $0 \leq r \leq a$ can be represented by the (mean) convergent series

$$
f(r)=\sum_{n=1}^{\infty} c_{n} j_{l}\left(k_{l, n} r\right)
$$

where

$$
c_{n}=\frac{2}{a^{3}\left[j_{l+1}\left(k_{l, n} a\right)\right]^{2}} \int_{0}^{a} j_{l}\left(k_{l, n} r\right) f(r) r^{2} d r .
$$

As with ordinary Bessel functions, when $a \rightarrow \infty$ and the homogeneous boundary conditions become $\left|R_{l}(0)\right|<\infty$ and $\lim _{r \rightarrow \infty}\left|R_{l}(r)\right|<\infty$, the eigenfunction solutions of (11.4.21) are $j_{l}(k r)$ where $k$ is now a continuous variable with range $0 \leq k<\infty$. The orthogonality/normalization statement becomes

$$
\int_{0}^{\infty} j_{l}(k r) j_{l}\left(k^{\prime} r\right) r^{2} d r=\frac{\pi}{2 k^{2}} \delta\left(k-k^{\prime}\right),
$$


the closure relation is

$$
\int_{0}^{\infty} j_{l}(k r) j_{l}\left(k r^{\prime}\right) k^{2} d k=\frac{\pi}{2 r^{2}} \delta\left(r-r^{\prime}\right),
$$

and any function $f(r)$ that is square integrable with respect to $r^{2}$ on $0 \leq r<\infty$ can be represented by the Fourier Bessel transform

$$
f(r)=\sqrt{\frac{2}{\pi}} \int_{0}^{\infty} j_{l}(k r) F(k) k^{2} d k
$$

where

$$
F(k)=\sqrt{\frac{2}{\pi}} \int_{0}^{\infty} j_{l}(k r) f(r) r^{2} d r .
$$

\subsubsection{Applications: Spherical Waves}

\section{Particle in a Spherical Box}

The independent particle "shell model" of the atomic nucleus postulates that each nucleon in a medium to large nucleus experiences an effective central potential due to the sum of all of the pair-wise interactions it has with the other nucleons. In the simplest version, the central potential is taken to be $V(r)=-V_{0}$ for $0 \leq r<R$ and, to insure that the nucleons cannot escape, infinite at $r=R$ where $R$ is the radius of the nucleus in question. Under these circumstances, the time-independent Schrödinger equation for each nucleon is just the Helmholtz equation

$$
\nabla^{2} \psi+k^{2} \psi=0 \text { where } k^{2}=\frac{2 m}{\hbar^{2}} \sqrt{E-V_{0}}
$$

and its solutions are subject to the boundary conditions $\lim _{r \rightarrow 0}|\psi(r, \theta, \varphi)|<\infty$ and $\psi(R, \theta, \varphi)=0$. From the foregoing analysis, we see immediately that the (unnormalized) energy eigenfunctions are $\psi_{l m n}(r, \theta, \varphi)=j_{l}\left(k_{l, n} r\right) Y_{l}^{m}(\theta, \varphi)$ and the corresponding energy levels are $E_{l n}=\frac{\hbar^{2}}{2 m} k_{l, n}^{2}-V_{0}$ where $k_{l, n}=\frac{z_{l, n}}{R}$. Notice that the levels are $(2 l+1)$-fold degenerate. Allowing for the spin of the nucleons, they are in fact $2(2 l+1)$ fold degenerate. It is this degeneracy that produces the so-called "magic numbers" of nucleons associated with increased nuclear stability and was the basis for developing and elaborating on this simple model.

\section{Acoustic Radiation}

The energy eigenfunctions of the preceding application will, with one modification, also describe the normal modes of sound waves in a gas that is contained in a spherical 
cavity. The one modification is due to a change in boundary condition at the wall of the container: we now have $\left.\boldsymbol{n} \cdot \nabla \psi(r, \theta, \varphi, t)\right|_{r=R}=\left.\frac{\partial \psi}{\partial r}\right|_{r=R}=0$ and so $k_{l n}=\frac{w_{l n}}{R}$ where $w_{l n}$ is the nth zero of $j_{l}^{\prime}(x)$. Thus, the waves are described by the superposition

$$
\psi(r, \theta, \varphi, t)=\sum_{n=1}^{\infty} \sum_{l=0}^{\infty} \sum_{m=-l}^{l} j_{l}\left(k_{l n} r\right) Y_{l}^{m}(\theta, \varphi)\left[a_{l m n} \cos \omega_{l n} t+b_{l m n} \sin \omega_{l n} t\right]
$$

where $\omega_{l n}=c k_{l n}$. The coefficients are determined by the initial conditions $\psi(r, \theta, \varphi, 0)=$ $u_{0}(r, \theta, \varphi)$ and $\left.\frac{\partial \psi}{\partial t}\right|_{t=0}=v_{0}(r, \theta, \varphi)$. Specifically,

$$
\begin{gathered}
a_{l m n}=\frac{2}{R^{3}\left[j_{l+1}\left(k_{l n} R\right)\right]^{2}} \int_{0}^{R} \int_{0}^{\pi} \int_{0}^{2 \pi} u_{0}(r, \theta, \varphi) j_{l}\left(k_{l n} r\right)\left(Y_{l}^{m}(\theta, \varphi)\right)^{\star} r^{2} d r \sin \theta d \theta d \varphi, \\
b_{l m n}=\frac{2}{\omega_{l n} R^{3}\left[j_{l+1}\left(k_{l n} R\right)\right]^{2}} \int_{0}^{R} \int_{0}^{\pi} \int_{0}^{2 \pi} v_{0}(r, \theta, \varphi) j_{l}\left(k_{l n} r\right)\left(Y_{l}^{m}(\theta, \varphi)\right)^{\star} r^{2} d r \sin \theta d \theta d \varphi .
\end{gathered}
$$

To describe travelling rather than standing waves we use spherical Hankel functions in place of the spherical Bessel functions. Thus, an $l$ th partial wave in a superposition will be either

$$
\psi_{l m}(r, t)=h_{l}^{(1)}(k r) Y_{l}^{m}(\theta, \varphi) e^{-i \omega t} \text { or } \psi_{l m}(r, t)=h_{l}^{(2)}(k r) Y_{l}^{m}(\theta, \varphi) e^{-i \omega t}
$$

depending on whether we want the wave to look asymptotically like an outgoing or an incoming wave. To understand this point, we need look no further than equations (11.4.17) and (11.4.18) which give the large argument behaviour of $h_{l}^{(1)}(x)$ and $h_{l}^{(2)}(x)$. With the choice of $e^{-i \omega t}$ to describe the time dependence of waves generated with frequency $\omega$, the $h_{l}^{(1)}(k r)$ combination will have the limit

$$
\psi_{l m}(r, t) \sim(-i)^{l+1} \frac{e^{i(k r-\omega t)}}{k r} Y_{l}^{m}(\theta, \varphi) \text { as } r \rightarrow \infty
$$

which represents an outgoing spherical wave. The $h_{l}^{(2)}(k r)$ combination on the other hand has the limit

$$
\psi_{l m}(r, t) \sim i^{l+1} \frac{e^{-i(k r+\omega t)}}{k r} Y_{l}^{m}(\theta, \varphi) \text { as } r \rightarrow \infty
$$

which corresponds to an incoming spherical wave. (If we had chosen an $e^{+i \omega t}$ time dependence, the incoming and outgoing roles would be reversed.)

Suppose that we have a monochromatic (fixed $\omega$ ) source that generates at $r=a$ sound waves of the form

$$
\psi(r, \theta, \varphi, t)=F(\theta, \varphi) e^{-i \omega t}
$$


where $F(\theta, \varphi)$ is a known function determined by the nature of the source. For $r>a$, the radiation is a superposition of outgoing waves with the same frequency,

$$
\psi(r, \theta, \varphi, t)=e^{-i \omega t} \sum_{l=0}^{\infty} \sum_{m=-l}^{l} a_{l m} h_{l}^{(1)}(k r) Y_{l}^{m}(\theta, \varphi), \quad k=\frac{\omega}{c} .
$$

The coefficients $a_{l m}$ can be determined from the boundary condition at $r=a$ :

$$
F(\theta, \varphi)=\sum_{l=0}^{\infty} \sum_{m=-l}^{l} a_{l m} h_{l}^{(1)}(k a) Y_{l}^{m}(\theta, \varphi)
$$

and so

$$
a_{l m}=\frac{1}{h_{l}^{(1)}(k a)} \int_{0}^{2 \pi} \int_{0}^{\pi}\left(Y_{l}^{m}(\theta, \varphi)\right)^{\star} F(\theta, \varphi) \sin \theta d \theta d \varphi .
$$

As an example let us assume that the waves are produced by a "split-sphere" antenna for which

$$
F(\theta, \varphi) \equiv F(\theta)=\left\{\begin{array}{cc}
f_{0} & 0<\theta<\frac{\pi}{2} \\
-f_{0} & \frac{\pi}{2}<\theta<\pi
\end{array} .\right.
$$

The waves will share the azimuthal symmetry of the antenna and so we need only retain the $m=0$ terms in the superpositions. Thus,

$$
\psi(r, \theta, \varphi, t)=\psi(r, \theta, t)=e^{-i \omega t} \sum_{l=0}^{\infty} a_{l} h_{l}^{(1)}(k r) P_{l}(\cos \theta)
$$

with

$$
F(\theta)=\sum_{l=0}^{\infty} a_{l} h_{l}^{(1)}(k a) P_{l}(\cos \theta)
$$

and

$$
a_{l}=\frac{2 l+1}{2} \frac{1}{h_{l}^{(1)}(k a)}\left[f_{o} \int_{0}^{\frac{\pi}{2}} P_{l}(\cos \theta) \sin \theta d \theta-f_{0} \int_{\frac{\pi}{2}}^{\pi} P_{l}(\cos \theta) \sin \theta d \theta\right] .
$$

We have evaluated the term in square brackets in an earlier example. It yields zero for even values of $l$ and

$$
a_{l}=(-1)^{\frac{l-1}{2}} \frac{f_{0}}{h_{l}^{(1)}(k a)} \frac{(2 l+1)(l+1)(l-1) !}{2^{l+1}\left[\left(\frac{l+1}{2}\right) !\right]^{2}}
$$

for odd values of $l$. Therefore, our final solution is

$$
\psi(r, \theta, \varphi, t)=f_{0} e^{-i \omega t} \sum_{l=1,3, \ldots}^{\infty}(-1)^{\frac{l-1}{2}} \frac{(2 l+1)(l+1)(l-1) !}{2^{l+1}\left[\left(\frac{l+1}{2}\right) !\right]^{2}} \frac{h_{l}^{(1)}(k r)}{h_{l}^{(1)}(k a)} P_{l}(\cos \theta) .
$$


When we are far from the source, we can replace $h_{l}^{(1)}(k r)$ by its asymptotic form. The wave then becomes

$$
\psi(r, \theta, \varphi, t) \sim f(\theta, \varphi) \frac{e^{i(k r-\omega t)}}{r}
$$

where

$$
f(\theta, \varphi)=\frac{1}{k} \sum_{l=0}^{\infty} \sum_{m=-l}^{l} a_{l m}(-i)^{l+1} Y_{l}^{m}(\theta, \varphi) .
$$

For the special case of waves produced by a split-sphere antenna, we have

$$
f(\theta, \varphi)=-\frac{f_{0}}{k} \sum_{l=1,3,5, \ldots}^{\infty} \frac{(2 l+1)(l+1)(l-1) !}{2^{l+1}\left[\left(\frac{l+1}{2}\right) !\right]^{2}} \frac{1}{h_{l}^{(1)}(k a)} P_{l}(\cos \theta) .
$$

The relevance of the function $f(\theta, \varphi)$ becomes clear when we realize that the energy density at a point $r$ associated with a monochromatic wave $\psi(r, t)$ is proportional to $|\psi(r, t)|^{2}$. Thus, the energy flux in the $(\theta, \varphi)$ direction is proportional to $c|\psi(r, \theta, \varphi, t)|^{2}$ which means when we are far from the source

$$
\text { the energy flux } \propto c \frac{|f(\theta, \varphi)|^{2}}{r^{2}} .
$$

To obtain the rate of energy flow through solid angle $d \Omega=\sin \theta d \theta d \varphi$ in the direction $(\theta, \varphi)$ at $\boldsymbol{r}$, we multiply the flux by the subtending area $r^{2} d \Omega$. The two factors of $r^{2}$ cancel and we find that the wave energy per unit time (or power) per unit solid angle is

$$
\frac{d P}{d \Omega} \propto c|f(\theta, \varphi)|^{2} .
$$

As we will see, the physical significance which this relation attaches to $f(\theta, \varphi)$ becomes particularly important in the analysis of scattering of waves.

\section{Expansion of a Plane Wave in Spherical Waves}

For a plane wave $e^{i(\boldsymbol{k} \cdot \boldsymbol{r}-\omega t)}$ propagating in the $z$ direction, $\boldsymbol{k}$ will be parallel to the $z$-axis and so the spatial part of the wave will be $u(r)=e^{i k \cdot r}=e^{i k r \cos \theta}$. The spatial part must also be a solution of the Helmholtz equation, $\nabla^{2} u+k^{2} u=0$, and so must have a representation in spherical coordinates of the form

$$
u(\boldsymbol{r})=\sum_{l=0}^{\infty} \sum_{m=-l}^{l}\left\{\begin{array}{l}
j_{l}(k r) \\
n_{l}(k r)
\end{array}\right\} Y_{l}^{m}(\theta, \varphi)
$$

However, because it has no $\varphi$ dependence and because it is finite at the origin, this simplifies to

$$
u(\boldsymbol{r})=e^{i k r \cos \theta}=\sum_{l=0}^{\infty} c_{l} j_{l}(k r) P_{l}(\cos \theta)
$$


which is a Fourier-Legendre series with coefficients given by

$$
c_{l} j_{l}(k r)=\frac{2 l+1}{2} \int_{0}^{\pi} e^{i k r \cos \theta} P_{l}(\cos \theta) \sin \theta d \theta .
$$

To simplify the evaluation of the coefficients, we introduce the variables $x=\cos \theta$ and $y=k r$. We then have

$$
e^{i x y}=\sum_{l=0}^{\infty} c_{l} j_{l}(y) P_{l}(x) \text { with } c_{l} j_{l}(y)=\frac{2 l+1}{2} \int_{-1}^{1} e^{i x y} P_{l}(x) d x .
$$

We shall go through two rather different evaluations of the coefficients since both offer insights into the tricks of manipulating special functions.

The first evaluation makes use of recurrence relations. Since

$$
P_{l}(x)=\frac{1}{2 l+1}\left[P_{l+1}^{\prime}(x)-P_{l+1}^{\prime}(x)\right]
$$

we find

$$
\begin{aligned}
c_{l} j_{l}(y) & =\frac{1}{2} \int_{-1}^{1} e^{i x y}\left[P_{l+1}^{\prime}(x)-P_{l-1}^{\prime}(x)\right] d x \\
& =\frac{i y}{2} \int_{-1}^{1} e^{i y x}\left[P_{l-1}(x)-P_{l+1}(x)\right] d x \\
& =i y\left[\frac{1}{2 l-1} c_{l-1} j_{l-1}(y)-\frac{1}{2 l+3} c_{l+1} j_{l+1}(y)\right] .
\end{aligned}
$$

However, we already know a unique relationship that links these three spherical Bessel functions, the recurrence relation

$$
j_{l}(y)=\frac{y}{2 l+1}\left[j_{l+1}(y)+j_{l-1}(y)\right] .
$$

Therefore, consistency requires that

$$
c_{l}=i\left(\frac{2 l+1}{2 l-1}\right) c_{l-1}=-i\left(\frac{2 l+1}{2 l+3}\right) c_{l+1} \text {. }
$$

Applied $l$ times, this relation gives us $c_{l}=i^{l}(2 l+1) c_{0}$ and

$$
e^{i x y}=c_{0} \sum_{l=0}^{\infty} i^{l}(2 l+1) j_{l}(y) P_{l}(x) .
$$

The final step sets $y=0$. Then, since $j_{0}(0)=1$ and $j_{l}(0)=0$ for $l>0$, we deduce that $c_{0}=1$ and the spherical wave expansion of a plane wave is

$$
e^{i k z}=e^{i k r \cos \theta}=\sum_{l=0}^{\infty} i^{l}(2 l+1) j_{l}(k r) P_{l}(\cos \theta) .
$$


This, like the expansion of the monochromatic wave in the preceding example, is often referred to as a partial wave expansion.

The second approach to evaluating the expansion coefficients makes use of Rodrigues' formulas. Starting with the formula for $P_{l}(x)$, we have

$$
\begin{aligned}
c_{l} j_{l}(y) & =\frac{2 l+1}{2} \frac{1}{2^{l} l !} \int_{-1}^{1} e^{i x y} \frac{d^{l}}{d x^{l}}\left(x^{2}-1\right)^{l} d x \\
& =\frac{2 l+1}{2^{l+1} l !}(-i y)^{l} \int_{-1}^{1} e^{i x y}\left(x^{2}-1\right)^{l} d x \equiv \frac{2 l+1}{2^{l+1} l !}(-i y)^{l} I_{l}
\end{aligned}
$$

where we have performed $l$ integrations by parts. The integral $I_{l}$ can be subjected to further integrations by parts. Substituting $\frac{d}{d x} \frac{e^{i x y}}{i y}$ for $e^{i x y}$, we have

$$
\begin{aligned}
I_{l} & =\int_{-1}^{1}\left(\frac{d}{d x} \frac{e^{i x y}}{i y}\right)\left(x^{2}-1\right)^{l} d x \\
& =(-2 l) \int_{-1}^{1} \frac{e^{i x y}}{i y} x\left(x^{2}-1\right)^{l-1} d x \\
& =(2 l) \frac{1}{y} \frac{d}{d y} \int_{-1}^{1} e^{i x y}\left(x^{2}-1\right)^{l} d x .
\end{aligned}
$$

Performing a second integration by parts, this becomes

$$
I_{l}=(2 l) 2(l-1) \frac{1}{y} \frac{d}{d y} \frac{1}{y} \frac{d}{d y} \int_{-1}^{1} e^{i x y}\left(x^{2}-1\right)^{l} d x
$$

from which we deduce that $l$ integrations will yield

$$
I_{l}=2^{l} l !\left(\frac{1}{y} \frac{d}{d y}\right)^{l} \int_{-1}^{1} e^{i x y} d x=2^{l+1} l !\left(\frac{1}{y} \frac{d}{d y}\right)^{l}\left(\frac{\sin y}{y}\right)
$$

But we know that

$$
j_{l}(y)=(-y)^{l}\left(\frac{1}{y} \frac{d}{d y}\right)^{l}\left(\frac{\sin y}{y}\right) .
$$

Therefore, returning to our Fourier-Legendre coefficients we have

$$
c_{l} j_{l}(y)=\frac{2 l+1}{2^{l+1} l !}(-i y)^{l} 2^{l+1} l ! \frac{j_{l}(y)}{(-y)^{l}}=(2 l+1) i^{l} j_{l}(y),
$$

or

$$
c_{l}=(2 l+1) i^{l}
$$


Thus, we again find that the partial wave expansion of a plane wave is

$$
e^{i k z}=e^{i k r \cos \theta}=\sum_{l=0}^{\infty}(2 l+1) i^{l} j_{l}(k r) P_{l}(\cos \theta) .
$$

Notice that we have derived an interesting integral relationship linking spherical Bessel functions and Legendre polynomials

$$
j_{l}(y)=\frac{1}{2 i^{l}} \int_{-1}^{1} e^{i x y} P_{l}(x) d x .
$$

Since this looks like an integral transform, one expects that there must be an inverse relation as well. To find it, one requires the orthogonality and normalization condition

$$
\int_{-\infty}^{\infty} j_{l}(x) j_{m}(x) d x=\frac{\pi}{2 l+1} \delta_{l, m} .
$$

Then, treating the expansion of $e^{i x y}$ as a Fourier-Bessel series, we find

$$
P_{l}(x)=\frac{1}{\pi i^{l}} \int_{-\infty}^{\infty} e^{i x y} j_{l}(y) d y .
$$

\section{Scattering of Waves by a Sphere}

We shall now make use of the partial wave expansions of the two preceding examples to analyse what happens when a plane wave impinges on a spherical object. We will centre the object at the origin and take its radius to be $R$. The plane wave is assumed to be propagating in the $z$ direction with unit amplitude and so it is described by

$$
\psi_{i n c}(\boldsymbol{r}, t)=e^{i(\boldsymbol{k} \cdot \boldsymbol{r}-\omega t)}=e^{i(k r \cos \theta-\omega t)} .
$$

Collision with the obstacle will result downstream in an outgoing spherical scattered wave superimposed on the incident plane wave. Since the incident wave is monochromatic, we can assume quite reasonably that the scattered wave will be monochromatic too and with the same frequency. Thus, the downstream wave will be of the form

$$
\psi(\boldsymbol{r}, t)=e^{i(k r \cos \theta-\omega t)}+\psi_{s c}(\boldsymbol{r}, t)
$$

where $\psi_{s c}$ is the superposition

$$
\psi_{s c}(\boldsymbol{r}, t)=e^{-i \omega t} \sum_{l=0}^{\infty} \sum_{m=-l}^{l} a_{l m} h_{l}^{(1)}(k r) Y_{l}^{m}(\theta, \varphi) .
$$




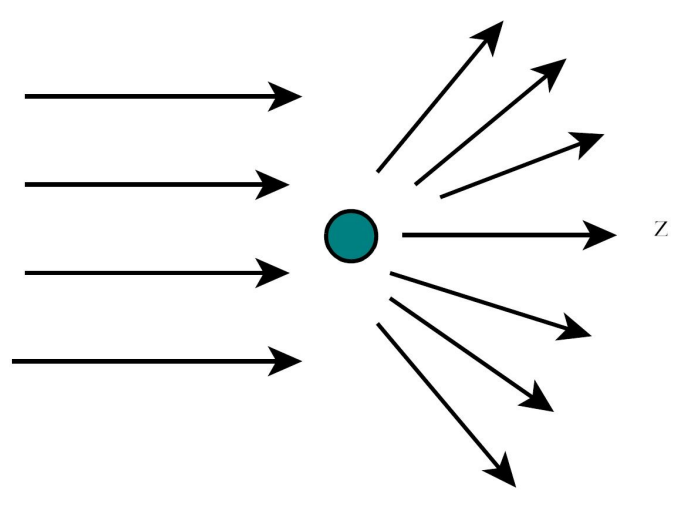

Far from the obstacle, $r \rightarrow \infty$ and the scattered wave assumes the asymptotic form

$$
\psi_{s c}(\boldsymbol{r}, t) \sim f(\theta, \varphi) \frac{e^{i(k r-\omega t)}}{r} \text { with } f(\theta, \varphi)=\frac{1}{k} \sum_{l=0}^{\infty} \sum_{m=-l}^{l}(-i)^{l+1} a_{l m} Y_{l}^{m}(\theta, \varphi) .
$$

In this context, the function $f(\theta, \varphi)$ is called the scattering amplitude and because of its connection to the power per unit solid angle associated with the scattered wave, it can be determined from what we call the differential cross-section for scattering into solid angle $d \Omega$ in direction $(\theta, \varphi)$ :

$$
d \sigma \equiv \frac{\text { power of scattered wave in solid angle } d \Omega}{\text { power of incident wave in unit area }}=\frac{c|f(\theta, \varphi)|^{2}}{c\left|e^{i(k z-\omega t)}\right|^{2}},
$$

or

$$
\frac{d \sigma}{d \Omega}=|f(\theta, \varphi)|^{2} .
$$

The problem as we have set it up thus far has azimuthal symmetry about the $\mathrm{z}$ axis and so, unless the boundary condition at $r=a$ breaks this symmetry, the solution should be independent of $\varphi$. This will certainly be the case if the boundary condition is homogeneous as happens for sound waves scattering from a rigid sphere and for "hard sphere scattering" in quantum mechanics which have the boundary conditions $\left.\frac{\partial \psi}{\partial r}\right|_{r=R}=0$ and $\psi(R, \theta, \varphi)=0$, respectively. Therefore, for such problems, we can write

$$
\psi_{s c}(\boldsymbol{r}, t)=e^{-i \omega t} \sum_{l=0}^{\infty} a_{l} h_{l}^{(1)}(k r) P_{l}(\cos \theta)
$$

and

$$
\psi(\boldsymbol{r}, t)=e^{i(k r \cos \theta-\omega t)}+\psi_{s c}(\boldsymbol{r}, t)=e^{-i \omega t} \sum_{l=0}^{\infty}\left[(2 l+1) i^{l} j_{l}(k r)+a_{l} h_{l}^{(1)}(k r)\right] P_{l}(\cos \theta)
$$

where, in the last equation, we have used the partial wave expansion of the plane wave derived in the preceding application. Imposing the boundary condition appropriate to 
sound waves on this superposition, we find

$$
\sum_{l=0}^{\infty}\left[(2 l+1) i^{l} j_{l}^{\prime}(k R)+a_{l} h_{l}^{(1) \prime}(k R)\right] P_{l}(\cos \theta)=0
$$

and hence,

$$
a_{l}=-(2 l+1) i^{l} \frac{j_{l}^{\prime}(k R)}{h_{l}^{(1) \prime}(k R)} .
$$

This completely determines the scattered wave and, using its asymptotic form for $r \gg a$, the scattering amplitude as well. Specifically, we have

$$
f(\theta, \varphi) \equiv f(\theta)=\frac{1}{k} \sum_{l=0}^{\infty} a_{l}(-i)^{l+1} P_{l}(\cos \theta)=\frac{i}{k} \sum_{l=0}^{\infty}(2 l+1) \frac{j_{l}^{\prime}(k R)}{h_{l}^{\prime}(k R)} P_{l}(\cos \theta) .
$$

This can be simplified by introducing a parameter $\delta_{l}(k)$ that is defined by the equation

$$
\tan \delta_{l}(k) \equiv \frac{j_{l}^{\prime}(k R)}{n_{l}^{\prime}(k R)}
$$

The reason for this somewhat unusual looking choice becomes apparent from two considerations. First, the factor

$$
i \frac{j_{l}^{\prime}(k R)}{h_{l}^{\prime}(k R)}=i \frac{j_{l}^{\prime}(k R)}{j_{l}^{\prime}(k R)+i n_{l}^{\prime}(k R)}=\frac{1}{\frac{n_{l}^{\prime}(k R)}{j_{l}^{\prime}(k R)}-i}=\frac{1}{\operatorname{cotan} \delta_{l}(k)-i}=e^{i} \delta_{l}(k) \sin \delta_{l}(k)
$$

and so the scattering amplitude becomes

$$
f(\theta)=\frac{1}{k} \sum_{l=0}^{\infty}(2 l+1) e^{i \delta_{l}(k)} \sin \delta_{l}(k) P_{l}(\cos \theta) .
$$

Second, when we express the asymptotic form of the wave in terms of $\delta_{l}$ we find

$$
\begin{aligned}
\psi(\boldsymbol{r}, t) & =e^{i(k r \cos \theta-\omega t)}+\psi_{s c}(\boldsymbol{r}, t) \\
& \sim e^{-i \omega t} \sum_{l=0}^{\infty}(2 l+1)\left[i^{l} \frac{\cos \left(k r-(l+1) \frac{\pi}{2}\right)}{k r}+\frac{e^{i \delta_{l}(k)} \sin \delta_{l}(k)}{k} \frac{e^{i k r}}{r}\right] P_{l}(\cos \theta) \\
& =\frac{e^{-i \omega t}}{2 i k} \sum_{l=0}^{\infty}(2 l+1)\left[\frac{e^{i k r}}{r}+(-1)^{l+1} \frac{e^{-i k r}}{r}+\left(e^{2 i \delta_{l}(k)}-1\right) \frac{e^{i k r}}{r}\right] P_{l}(\cos \theta) \\
& =\frac{e^{-i \omega t}}{2 i k} \sum_{l=0}^{\infty}(2 l+1)\left[e^{2 i \delta_{l}(k)} \frac{e^{i k r}}{r}+(-1)^{l+1} \frac{e^{-i k r}}{r}\right] P_{l}(\cos \theta)
\end{aligned}
$$

which tells us that the effect of the sphere has been to shift the phase of the outgoing wave by an amount $2 \delta_{l}(k)$. Thus, $\delta_{l}(k)$ has an intuitively satisfying interpretation and one that is the source of its name: $\delta_{l}(k)$ is called the lth partial wave phase shift. 
Notice that the total scattering cross section which is obtained by integrating $\frac{d \sigma}{d \Omega}$ over all solid angles has a particularly simple expression in terms of phase shifts. The integration gives

$$
\sigma=(2 \pi) \int_{0}^{\pi}|f(\theta)|^{2} \sin \theta d \theta=\frac{4 \pi}{k^{2}} \sum_{l=0}^{\infty} \sin ^{2} \delta_{l}(k)=\sum_{l=0}^{\infty} \sigma_{l} \text { where } \sigma_{l}=\frac{4 \pi}{k^{2}} \sin ^{2} \delta_{l}(k) .
$$

These measures of the scattering can be easily estimated only in the "large wavelength limit", $k R \ll 1$ or $\lambda \gg R$. From the small argument behaviour of the spherical Bessel and Neumann functions we have

$$
\tan \delta_{l}(k) \simeq \frac{l}{l+1} \frac{\left(2^{l} l !\right)^{2}}{(2 l) !(2 l+1) !}(k R)^{2 l+1} \text { for } l \geq 1,
$$

and

$$
\tan \delta_{0}(k)=\frac{j_{0}^{\prime}(k R)}{n_{0}^{\prime}(k R)}=\frac{j_{1}(k R)}{n_{1}(k R)} \simeq-\frac{(k R)^{3}}{3} .
$$

Therefore, $\delta_{l}(k)$ is itself $\ll 1$, and so

$$
e^{i \delta_{l}(k)} \sin \delta_{l}(k) \simeq \delta_{l}(k) \simeq \tan \delta_{l}(k) .
$$

Moreover, $\delta_{l}(k)$ decreases rapidly with increasing $l>1$ so we need to retain only the first few partial waves. Thus,

$$
f(\theta) \simeq \frac{1}{k}\left[-\frac{(k R)^{3}}{3} P_{0}(\cos \theta)+3 \frac{(k R)^{3}}{6} P_{1}(\cos \theta)+\ldots\right] \simeq-\frac{k^{2} R^{3}}{3}\left[1-\frac{3}{2} \cos \theta\right]
$$

and

$$
\sigma \simeq \frac{5 \pi}{9} k^{4} R^{6}
$$

This formalism for the analysis of scattering problems is very general. The only thing that changes from problem to problem is the boundary condition at the obstacle and the consequent definition of the phase shift. For example, if instead of $\left.\frac{\partial \psi}{\partial r}\right|_{r=R}=0$ we had used the boundary condition for quantum mechanical hard sphere scattering, $\psi(R, \theta, \varphi)=0$, we would replace our definition of $\delta_{l}(k)$ above with the identity

$$
\tan \delta_{l}(k) \equiv \frac{j_{l}(k R)}{n_{l}(k R)}
$$

but everything else would be unchanged.

\subsection{The Classical Orthogonal Polynomials}

\subsubsection{The Polynomials and Their Properties}

We have completed a study of the special functions that arise from the solution of the Laplace and Helmholtz equations in spherical and cylindrical coordinates. However, 
this is not an inclusive set: there are further special functions that arise, for example, in quantum mechanics. One such is the set of Hermite polynomials which we encountered in Chapter 9 as the solutions of a DE that we attributed to a one-dimensional harmonic oscillator. Others include the Laquerre polynomials (which feature in the theory of the hydrogen atom and of the three-dimensional harmonic oscillator), the Tchebichef polynomials and the Gegenbauer polynomials. Together with the Legendre polynomials, they are examples of what have come to be known as the classical orthogonal polynomials.

A common feature of these functions is that each class of polynomials is orthogonal on an interval $a \leq x \leq b$ with respect to a weight function $\rho(x)$. This could be used to define the polynomials since they could be generated from a Schmidt orthogonalization process applied to the monomials $1, x, x^{2}, x^{3}, \ldots$ However, there is a more elegant approach based on a second common feature: each class can be computed from a Rodrigues' formula.

We define a generalized Rodrigues' formula by means of the identity

$$
C_{n}(x)=\frac{1}{K_{n}} \frac{1}{\rho(x)} \frac{d^{n}}{d x^{n}}\left[\rho(x) s^{n}(x)\right]
$$

where

- $K_{n}$ is a standardizing constant that is chosen by convention

- $\quad \rho(x)$ is a real, positive (weight) function that is integrable on the interval $a \leq x \leq b$

- $s(x)$ is a polynomial of degree $\leq 2$ with real roots and satisfies the boundary conditions

$$
\rho(a) s(a)=\rho(b) s(b)=0 \text {. }
$$

In addition, we require that $C_{1}(x)$ be a first-degree polynomial.

We know of course that Legendre polynomials satisfy these constraints with

$$
a=-1, \quad b=1, \quad \rho(x)=1, K_{n}=2^{n} n !, \quad s(x)=x^{2}-1 \text { and } C_{1}=x .
$$

The question is, do all $C_{n}(x)$ that satisfy these requirements comprise a set of polynomials of degree $n$ that are orthogonal with respect to weight function $\rho(x)$ on the interval $a \leq x \leq b$ ? As we will now prove, the answer is yes.

We start by noting that setting $n=1$ in (11.5.1) gives us

$$
C_{1}(x) \rho(x) K_{1}=\frac{d}{d x}[\rho(x) s(x)] \text { or } s(x) \frac{d \rho}{d x}=\rho(x)\left(C_{1}(x)-\frac{d s}{d x}\right) .
$$

Next, we calculate $\frac{d}{d x}\left[\rho(x) s^{n}(x) p_{k}\right]$ where $p_{k}(x)$ is an arbitrary polynomial of degree $k$ :

$$
\begin{aligned}
\frac{d}{d x}\left[\rho(x) s^{n}(x) p_{k}(x)\right] & =\frac{d \rho}{d x} s^{n} p_{k}+\rho n s^{n-1} \frac{d s}{d x} p_{k}+\rho s^{n} \frac{d p_{k}}{d x} \\
& =\rho\left(K_{1} C_{1}-\frac{d s}{d x}\right) s^{n-1} p_{k}+n \rho s^{n-1} \frac{d s}{d x} p_{k}+\rho s^{n}(x) \frac{d p_{k}}{d x}
\end{aligned}
$$




$$
=\rho(x) s^{n-1}(x)\left[\left(K_{1} C_{1}(x)+(n-1) \frac{d s}{d x}\right) p_{k}(x)+s(x) \frac{d p_{k}}{d x}\right]
$$

where we have used (11.5.3) to obtain the second line of the calculation. Since $s(x)$ is of degree $\leq 2, \frac{d s}{d x}$ is of degree $\leq 1$, and $\frac{d p_{k}}{d x}$ is of degree $k-1$, the term in square brackets on the right hand side is a polynomial of degree $\leq k+1$. Denoting the latter by $q_{k+1}(x)$, we write

$$
\frac{d}{d x}\left[\rho(x) s^{n}(x) p_{k}(x)\right]=\rho(x) s^{n-1}(x) q_{k-1}(x)
$$

from which we see that differentiating $m$ times will give us

$$
\frac{d^{m}}{d x^{m}}\left[\rho(x) s^{n}(x) p_{k}(x)\right]=\rho(x) s^{n-m}(x) q_{k+m}(x)
$$

where $q_{n+m}(x)$ is a polynomial of degree $\leq n+m$.

Notice that insertion of $p_{0}(x)=1$ into (11.5.4) gives us

$$
\frac{d^{m}}{d x^{m}}\left[\rho(x) s^{n}(x)\right]=\rho(x) s^{n-m}(x) q_{m}(x)
$$

and, because of the boundary conditions satisfied by $\rho(x) s(x)$, this means that all such derivatives with $m<n$ vanish at $x=a$ and $x=b$.

Moreover, if $m=n$, we have the derivative that appears in the Rodrigues' formula which allows us to assert that

$$
\begin{aligned}
C_{n}(x) & =q_{n}(x) \equiv \text { a polynomial of degree } \leq n \\
& =q_{n-1}(x)+a_{n} x^{n} \text { where } a_{n} \text { is a coefficient to be determined. }
\end{aligned}
$$

The next step involves the evaluation of the integral $\int_{a}^{b} p_{m}(x) C_{n}(x) \rho(x) d x$ where $p_{m}(x)$ is a polynomial of degree $m<n$. Invoking Rodrigues' formula for $C_{n}(x)$ and integrating by parts $n$ times, we find

$$
\begin{aligned}
\int_{a}^{b} p_{m}(x) C_{n}(x) \rho(x) d x & =\frac{1}{K_{n}} \int_{a}^{b} p_{m}(x) \frac{d^{n}}{d x^{n}}\left[\rho(x) s^{n}(x)\right] d x \\
& =\frac{(-1)^{n}}{K_{n}} \int_{a}^{b}\left[\rho(x) s^{n}(x)\right] \frac{d^{n}}{d x^{n}} p_{m}(x)=0
\end{aligned}
$$

where we have used the vanishing of derivatives of $\rho(x) s^{n}(x)$ at $x=a$ and $x=b$ to dispose of all integrated terms. we find

Now we make use of (11.5.5). Using it to replace one factor of $C_{n}(x)$ in $\int_{a}^{b} C_{n}^{2}(x) \rho(x) d x$ $N_{n} \equiv \int_{a}^{b} C_{n}^{2}(x) \rho(x) d x=\int_{a}^{b} q_{n-1}(x) C_{n}(x) d x+a_{n} \int_{a}^{b} x^{n} C_{n}(x) \rho(x) d x=a_{n} \int_{a}^{b} x^{n} C_{n}(x) \rho(x) d x$ 
by the preceding result. Since the left hand side cannot be zero, it follows that $a_{n}$ cannot be zero either and hence, that $C_{n}(x)$ is a polynomial of degree $n$. Moreover, using the Rodrigues' formula, we can evaluate its normalization integral:

$N_{n}=a_{n} \int_{a}^{b} x^{n} C_{n}(x) \rho(x) d x=\frac{a_{n}}{K_{n}} \int_{a}^{b} x^{n} \frac{d^{n}}{d x^{n}}\left[s^{n}(x) \rho(x)\right] d x=\frac{(-1)^{n} n ! a_{n}}{K_{n}} \int_{a}^{b} s^{n}(x) \rho(x) d x$

where we have performed $n$ integrations by parts to obtain the final line.

Finally, we substitute $C_{m}(x)$ for $p_{m}(x)$ in (11.5.6) to obtain

$$
\int_{a}^{b} C_{m}(x) C_{n}(x) \rho(x) d x=0 \text { for } m<n .
$$

Thus, not only does the generalized Rodrigues' formula define a sequence of polynomials, the sequence forms an orthogonal set of polynomials with respect to a weight $\rho(x)$ on the interval $a \leq x \leq b$.

At first sight, it might appear that this is a prescription for generating any number of distinct sets of orthogonal polynomials. In fact, there are only three classes of polynomial corresponding to the three possibilities for the degree of $s(x)$.

We can always define the polynomial $C_{1}(x)$ to be

$$
C_{1}(x)=-\frac{x}{K_{1}}
$$

since any other first-degree polynomial can be obtained from it by a linear transformation of the variable $x$. Substituting this into the Rodrigues' formula gives us a relationship between $\rho(x)$ and $s(x)$ :

$$
\frac{1}{\rho(x)} \frac{d \rho}{d x}=-\frac{1}{s(x)}\left(\frac{d s}{d x}+x\right) .
$$

Suppose that $s(x)$ is of zeroth-degree. Without loss of generality, we can set $s(x)=$ 1. Equation (11.5.9) becomes

$$
\frac{1}{\rho(x)} \frac{d \rho}{d x}=-x
$$

with solution $\rho(x)=$ const $e^{-\frac{x^{2}}{2}}$. The product $s(x) \rho(x)$ vanishes only at $x= \pm \infty$. Therefore, transforming $x$ by $\frac{x}{\sqrt{2}} \rightarrow x$ and setting the multiplicative constant equal to one, we conclude that a consistent set of parameters is

$$
s(x)=1, \quad \rho(x)=e^{-x^{2}}, \quad a=-\infty \text { and } b=\infty .
$$

With appropriate standardization, $K_{n}=(-1)^{n}$, the set $\left\{C_{n}(x)\right\}$ defined by these parameters consists of the Hermite polynomials $H_{n}(x)$. 
For the case of a first-degree $s(x)$, we set $s(x)=(x-\alpha)$. Equation (11.5.9) now yields the $\mathrm{DE}$

$$
\frac{1}{\rho(x)} \frac{d \rho}{d x}=-\frac{x+1}{x-\alpha}
$$

with solution (having set the multiplicative constant of integration equal to one)

$$
\rho(x)=(x-\alpha)^{-\alpha-1} e^{-x} .
$$

If $-\alpha-1 \equiv v>1$, then $s(x) \rho(x)$ vanishes at $x=\alpha$ and $x=\infty$ and $\rho(x)$ is integrable on $\alpha \leq x<\infty$. Thus, transforming $x$ by means of the substitution $x \rightarrow x+\alpha$ and ignoring multiplicative constants, we conclude that the appropriate parameters in this case are

$$
s(x)=x, \quad \rho(x)=x^{v} e^{-x}, \quad v>-1, \quad a=0, \quad b=\infty
$$

which correspond to $C_{1}(x)=\frac{1}{K_{1}}(v+1-x)$ and $\frac{1}{\rho(x)} \frac{d \rho}{d x}=\frac{v-x}{x}$. Used in the generalized Rodrigues' formula with $K_{n}=n$ ! , these generate the Laguerre polynomials $L_{n}^{v}(x)$.

Finally, if $s(x)$ is of second degree, we can assign it the quadratic form

$$
s(x)=(x-\alpha)(\beta-x), \quad \beta>\alpha .
$$

Equation (11.5.9) then reads

$$
\frac{1}{\rho(x)} \frac{d \rho}{d x}=\frac{(x-\alpha)-(\beta-x)-x}{(x-\alpha)(\beta-x)}
$$

which has the solution (ignoring multiplicative constants)

$$
\rho(x)=(x-\alpha)^{-\frac{\beta}{\beta-\alpha}}(\beta-x)^{-\frac{\alpha}{\beta-\alpha}} .
$$

Evidently $s(x) \rho(x)$ vanishes at $x=\alpha$ and $x=\beta$ and $\rho(x)$ is integrable on $\alpha \leq x \leq \beta$ provided that the exponents in $\rho(x)$ are both $>1$. This will be the case if we choose $\alpha=-1$ and $\beta=1$. Therefore, we can take our parameters to be

$$
s(x)=\left(1-x^{2}\right), \rho(x)=(x+1)^{\mu}(1-x)^{\nu}, \mu, v>-1, a=-1 \text { and } b=1
$$

which result in the redefinition

$$
C_{1}(x)=\frac{1}{K_{1}}[(\mu-v)-(\mu+v+2) x] \text { and } \frac{1}{\rho(x)} \frac{d \rho}{d x}=\frac{\mu(1-x)-v(1+x)}{1-x^{2}} .
$$

These produce the Jacobi polynomials $P_{n}^{(\mu, v)}(x)$ which have a number of special cases to which other names have been attached:

- the Gegenbauer polynomials $C_{n}^{\mu+\frac{1}{2}}(x)$ which correspond to $\mu=v$;

- the Legendre polynomials $P_{n}(x)$ which correspond to $\mu=v=0$;

- the Tchebichef polynomials of the first kind $T_{n}(x)$ which correspond to $\mu=v=$ $\frac{1}{2}$;

- the Tchebichef polynomials of the second kind $U_{n}(x)$ which correspond to $\mu=$ $v=-\frac{1}{2}$. 
We state without proof that any three consecutive orthogonal polynomials satisfy a recurrence relation of the form

$$
C_{n+1}(x)=\left(A_{n} x+B_{n}\right) C_{n}(x)-D_{n} C_{n-1}(x)
$$

where $A_{n}, B_{n}$ and $D_{n}$ are constants that depend only on $n$ and the class of polynomials under consideration. Indeed, one can show that

$$
A_{n}=\frac{a_{n+1}}{a_{n}}, \quad B_{n}=\frac{a_{n+1}}{a_{n}}\left(\frac{a_{n+1}^{\prime}}{a_{n+1}}-\frac{a_{n}^{\prime}}{a_{n}}\right), \quad D_{n}=\frac{N_{n}}{N_{n-1}} \frac{a_{n+1} a_{n-1}}{a_{n}^{2}}
$$

where, as before, $N_{n}$ is the normalization integral, $a_{n}$ is the coefficient of $x^{n}$, and $a_{n}^{\prime}$ is the coefficient of $x^{n-1}$, for the polynomial $C_{n}(x)$.

In addition, one can show that $C_{n}(x)$ satisfies the Sturm-Liouville DE

$$
\frac{d}{d x}\left(\rho(x) s(x) \frac{d C_{n}}{d x}\right)=-\lambda_{n} \rho(x) C_{n}(x)
$$

with

$$
\lambda_{n}=-n\left[K_{1} \frac{d C_{1}}{d x}+\frac{1}{2}(n-1) \frac{d^{2} s}{d x^{2}}\right] .
$$

The equation has regular singular points at the roots of $\rho(x) s(x)$ which means at $x=a$ and $x=b$. Thus, since the polynomials $C_{n}(x)$ are finite there, they must be eigenfunction solutions of the more general problem

$$
\frac{d}{d x}\left(\rho(x) s(x) \frac{d u}{d x}\right)=-\lambda \rho(x) u(x) \text { with }|u(b)|<\infty \text { and }|u(a)|<\infty .
$$

We list below the salient features of Hermite and Laguerre polynomials which, along with the Legendre polynomials, are the ones most frequently encountered.

\section{Hermite Polynomials:}

Rodrigues' formula

$$
H_{n}(x)=(-1)^{n} e^{x^{2}} \frac{d^{n}}{d x^{n}}\left(e^{-x^{2}}\right)
$$

Orthogonality and Normalization

$$
\int_{-\infty}^{\infty} H_{m}(x) H_{n}(x) e^{-x^{2}} d x=N_{n} \delta_{m, n}=\sqrt{\pi} 2^{n} n ! \delta_{m, n} .
$$

Differential equation

$$
\frac{d^{2}}{d x^{2}} H_{n}(x)-2 x \frac{d}{d x} H_{n}(x)+2 n H_{n}(x)=0
$$


Recurrence relation

$$
H_{n+1}(x)=2 x H_{n}(x)-2 n H_{n-1}(x)
$$

Series

$$
H_{n}(x)=\sum_{k=0}^{[n / 2]}(-1)^{k} \frac{n !}{(n-2 k) ! k !}(2 x)^{n-2 k}
$$

\section{Laguerre Polynomials:}

Rodrigue's formula

$$
L_{n}^{v}(x)=\frac{1}{n !} x^{-v} e^{x} \frac{d^{n}}{d x^{n}}\left(e^{-x} x^{v+n}\right)
$$

Orthogonality and Normalization

$$
\int_{0}^{\infty} L_{m}^{v}(x) L_{n}^{v}(x) x^{v} e^{-\chi} d x=N_{n} \delta_{m, n}=\frac{\Gamma(n+v+1)}{n !} \delta_{m, n}
$$

Differential equation

$$
x \frac{d^{2}}{d x^{2}} L_{n}^{v}(x)+(v+1-x) \frac{d}{d x} L_{n}^{v}(x)+n L_{n}^{v}(x)=0
$$

Recurrence relation

$$
(n+1) L_{n+1}^{v}(x)=(2 n+v+1-x) L_{n}^{v}(x)-(n+v) L_{n-1}^{v}(x)
$$

Series

$$
L_{n}^{v}(x)=\sum_{k=0}^{n}(-1)^{k} \frac{(n+v) !}{(n-k) !(v+k) ! k !} x^{k} \quad v>-1
$$

\subsubsection{Applications}

\section{The Quantum Mechanical Simple Harmonic Oscillator}

As mentioned earlier, the Hermite polynomials arise in the quantum mechanical description of simple harmonic oscillators. The potential energy of the oscillator is $V(z)=$ $\frac{1}{2} K z^{2}$ corresponding to a restoring force $\boldsymbol{F}=-\nabla V=-\frac{d}{d z} V \boldsymbol{k}=-K z \boldsymbol{k}$. Therefore, if we take the mass of the oscillating particle to be $m$, its time-independent Schrödinger equation is

$$
-\frac{\hbar^{2}}{2 m} \frac{d^{2} \psi}{d z^{2}}+\frac{1}{2} K z^{2} \psi(z)=E \psi(z)
$$


where $E$ is the total energy. In addition, the particle's wave function must be bounded and normalizable and so we require $\lim _{z \rightarrow \pm \infty}|\psi(z)|=0$.

Introducing the parameters

$$
x=\alpha z \text { with } \alpha^{4}=\frac{m K}{\hbar^{2}}=\frac{m^{2} \omega^{2}}{\hbar^{2}} \lambda=\frac{2 E}{\hbar}\left(\frac{m}{K}\right)^{\frac{1}{2}}=\frac{2 E}{\hbar \omega},
$$

where $\omega=\left(\frac{K}{m}\right)^{\frac{1}{2}}$ is the angular frequency of the corresponding classical oscillator, this equation becomes

$$
\frac{d^{2} u}{d x^{2}}+\left(\lambda-x^{2}\right) u(x)=0 \text { with } u(x)=\psi\left(\frac{x}{\alpha}\right)=\psi(z) .
$$

This is the same equation, (9.8.4), that we solved in Section 9.8.2 and found that $\lambda$ must be set equal to $2 n+1$ yielding a set of solutions $u_{n}(x) \propto e^{-\frac{x^{2}}{2}} H_{n}(x)$. Normalizing so that the wave functions give unit probability of finding the particle in the interval $-\infty<x<\infty$, we find

$$
u_{n}(x)=\pi^{-\frac{1}{4}}\left(2^{n} n !\right)^{-\frac{1}{2}} e^{-\frac{x^{2}}{2}} H_{n}(x)
$$

or,

$$
\psi_{n}(z)=\pi^{-\frac{1}{4}}\left(2^{n} n !\right)^{-\frac{1}{2}} e^{-\frac{\alpha^{2} z^{2}}{2}} H_{n}(\alpha z) .
$$

The corresponding energies are

$$
E_{n}=\left(n+\frac{1}{2}\right) \hbar \omega \quad n=0,1,2, \ldots
$$

Notice that the minimum or zero point energy is not zero but rather $E_{\min }=E_{0}=\frac{1}{2} \hbar \omega$. This is a quantum phenomenon connected with the uncertainty principle.

The three-dimensional harmonic oscillator has played an important role in understanding sub-atomic spectroscopy. In Section 11.4.2 we noted that the nuclear shell model assumes that the individual two-nucleon interactions in a medium to large nucleus sum to produce an effective central potential in which each nucleon moves independently. The next question is what functional form can be assigned to the central potential that will not only be simple to work with but will be reasonably realistic in the sense that its predictions agree at least qualitatively with experiment. The guess of Section 11.4.2, a "hard" or infinite spherical well, is very simple to work with but has only limited success. Far and away more successful is the almost as simple guess that the potential is that of a three-dimensional oscillator: $V(r)=-V_{0}+\frac{1}{2} K r^{2}$.

Another application of the oscillator potential is in understanding the bonding of quarks in sub-nuclear matter. Quarks interact with each other by means of gluon exchange which produces a force that increases with increasing separation and thus prevents the production of free quarks. At the level of non-relativistic quantum mechanics, an oscillator potential is a very convenient way of representing this effect and hence, of approximating the quark-quark interaction. 


\section{The Nuclear Shell Model}

We can absorb the constant part of the potential by redefining the zero of energy. Therefore, the (time-independent) Schrödinger equation that must be solved for a nucleon of mass $m$ in a potential $V(r)=\frac{1}{2} K r^{2}$ is

$$
-\frac{\hbar^{2}}{2 m} \nabla^{2} \psi+\frac{1}{2} K r^{2} \psi(\mathrm{r})=\mathrm{E} \psi(\mathrm{r})
$$

If we separate variables using Cartesian coordinates and the fact that $r^{2}=x^{2}+y^{2}+z^{2}$, this becomes three one-dimensional harmonic oscillator equations all with the same "spring constant" $K$ and with energies $E_{x}, E_{y}$, and $E_{z}$ that sum to $E: E=E_{x}+E_{y}+E_{z}$. Thus, the eigenfunction solutions of (11.5.33) that satisfy $\lim _{r \rightarrow \infty} \psi(r)=0$ may be expressed as

$$
\begin{aligned}
\psi_{N}(r) & =\psi_{n_{1}}(x) \psi_{n_{2}}(y) \psi_{n_{3}}(z) \\
& =\pi^{-\frac{3}{4}}\left(2^{N} n_{1} ! n_{2} ! n_{3} !\right)^{-\frac{1}{2}} e^{-\frac{\alpha^{2} r^{2}}{2}} H_{n_{1}}(\alpha x) H_{n_{2}}(\alpha y) H_{n_{3}}(\alpha z)
\end{aligned}
$$

and correspond to the energies

$$
E_{N}=E_{n_{1}}+E_{n_{2}}+E_{n_{3}}=\left(n_{1}+n_{2}+n_{3}+\frac{3}{2}\right) \hbar \omega=\left(N+\frac{3}{2}\right) \hbar \omega
$$

where

$$
\begin{array}{r}
N=n_{1}+n_{2}+n_{3}, n_{1}, n_{2}, n_{3}=0,1,2, \ldots . \\
\omega=\sqrt{\frac{K}{m}} \text { and } \alpha^{2}=\frac{m \omega}{\hbar} .
\end{array}
$$

Notice that the degeneracy of the eigenvalues increases fairly rapidly with increasing $N$. The ground state energy $E_{0}=\frac{3}{2} \hbar \omega$ is non-degenerate since $N=0$ implies uniquely $n_{1}=n_{2}=n_{3}=0$. However, the next energy $E_{1}=\frac{5}{2} \hbar \omega$ is three-fold degenerate because it is associated with the three distinct states associated with the triads $n_{1}=1, n_{2}=n_{3}=0, n_{2}=1, n_{1}=n_{3}=0$ and $n_{3}=1, n_{1}=n_{2}=0$. The $N=2$ energy is 6-fold degenerate and since in general there are $N+1$ choices for $n_{1}, \frac{N}{2}+1$ choices for $n_{2}$ and 1 choice for $n_{3}$, the degeneracy of the $N$ th level is

$$
d_{N}=\frac{(N+1)(N+2)}{2} .
$$

The existence of degeneracy always indicates that the eigenvalue problem possesses symmetry of some sort. In the present case, it is spherical symmetry and whenever we have encountered spherical symmetry before we have used it as a justification for using spherical rather than Cartesian coordinates. Doing so here, we set $\psi(\boldsymbol{r})=R(r) Y_{l}^{m}(\theta, \varphi)$ and substitute into (11.5.33) to find

$$
-\frac{\hbar^{2}}{2 m} \frac{1}{r} \frac{d^{2}}{d r^{2}}[r R(r)]+\left[\frac{l(l+1) \hbar^{2}}{2 m r^{2}}+\frac{1}{2} K r^{2}\right] R(r)=E R(r) .
$$


In quantum mechanics, unlike the description of classical waves, the $l$ has a physical association: it is a measure of the nucleon's orbital angular momentum. Evidently, it is a conserved quantity when there is spherical symmetry.

Making the replacement $u(r)=r R(r)$ equation (11.5.38) can be cast in the somewhat simpler form:

$$
\frac{d^{2} u}{d r^{2}}-\left[\alpha^{4} r^{2}+\frac{l(l+1)}{r^{2}}\right] u(r)=-\frac{2 m E}{\hbar^{2}} u(r) \text { where } \alpha^{2}=\frac{m \omega}{\hbar} .
$$

Solution of this DE requires a number of tricks from Section 9.8 . Our boundary conditions are boundedness at zero and infinity. Therefore, the first step is to examine the leading behaviour there and factor it out. As $r \rightarrow 0$, the DE assumes the limiting form

$$
\frac{d^{2} u}{d r^{2}}-\frac{l(l+1)}{r^{2}} u \simeq 0
$$

with solutions $r^{l+1}$ and $r^{-l}$. At the other extreme, as $r \rightarrow \infty$, the DE becomes

$$
\frac{d^{2} u}{d r^{2}}-\alpha^{4} r^{2} u \simeq 0
$$

with approximate solutions $e^{ \pm \frac{\alpha^{2} r^{2}}{2}}$. Therefore, to assure boundedness, we set

$$
u(r)=r^{l+1} e^{-\frac{\alpha^{2} r^{2}}{2}} v(r)
$$

Substituting this into the DE for $u(r)$ gives us

$$
\frac{d^{2} v}{d r^{2}}+2\left[\frac{l+1}{r}-\alpha^{2} r\right] \frac{d v}{d r}+\left[\frac{2 m E}{\hbar^{2}}-\alpha^{2}(2 l+3)\right] v=0
$$

which we expect to admit polynomial solutions. However, it is not readily apparent what kind of polynomials these may be. To save the effort of attempting a Frobenius solution, we make one further simplification by introducing the new independent variable $x=\alpha^{2} r^{2}$. Setting $y(x)=v(r)$, we obtain

$$
x \frac{d^{2} y}{d x^{2}}+\left(l+\frac{3}{2}-x\right) \frac{d y}{d x}+\frac{1}{4}\left(\frac{2 E}{\hbar \omega}-2 l-3\right) y=0
$$

which we can now compare with the DE's satisfied by classical orthogonal polynomials. A case by case comparison is quickly rewarded: this is precisely the form found in (11.5.25), the DE for Laguerre polynomials. Thus, $y(x)=L_{n}^{v}(x)$ with $n=\frac{1}{4}\left(\frac{2 E}{\hbar \omega}-2 l-3\right)$ and $v=l+\frac{1}{2}$. In other words, the quantized energies are

$$
E_{N}=E_{n l}=\left(2 n+l+\frac{3}{2}\right) \hbar \omega=\left(N+\frac{3}{2}\right) \hbar \omega, \quad N=0,1,2, \ldots
$$

in agreement with the conclusion (11.5.35) obtained with the use of Cartesian coordinates. 
The wave functions that correspond to these energies are

$$
\psi_{n l}(\boldsymbol{r})=N_{n l} r^{l} e^{-\frac{\alpha^{2} r^{2}}{2}} L_{n}^{l+\frac{1}{2}}\left(\alpha^{2} r^{2}\right) Y_{l}^{m}(\theta, \varphi)
$$

where $N_{n l}$ is a constant determined by normalizing to unity the probability of finding the nucleon somewhere in space. In fact, using (11.5.24) we find

$$
1=\int_{0}^{\infty} \int_{0}^{\pi} \int_{0}^{2 \pi}\left|\psi_{n l}(\boldsymbol{r})\right|^{2} r^{2} d r \sin \theta d \theta d \varphi=\frac{\left|N_{n l}\right|^{2}}{2} \frac{1}{\alpha^{2 l+3}} \frac{\Gamma(n+l+3 / 2)}{n !}
$$

and so,

$$
\psi_{n l}(\boldsymbol{r})=\left(\frac{2 \alpha^{3} n !}{\Gamma(n+l+3 / 2)}\right)^{\frac{1}{2}}(\alpha r)^{l} e^{-\frac{\alpha^{2} r^{2}}{2}} L_{n}^{l+\frac{1}{2}}\left(\alpha^{2} r^{2}\right) Y_{l}^{m}(\theta, \varphi) .
$$

Let us now check the degeneracy question. For $N=0$ there is only one possibility: $n=0$ and $l=0$. For $N=1$ there is again one possibility, $n=0$ and $l=1$, but for nonzero $l$ there is automatically a degeneracy of $2 l+1$ corresponding to the allowed values of $m$. Thus, we recover the same three-fold degeneracy that we found with Cartesian coordinates. Similarly, for $N=2$ we can have $n=0$ and $l=2$ plus $n=1$ and $l=0$ for a six-fold degeneracy and, in general, since $l$ can assume the values $N, N-2, N-4, \ldots$. down to 1 or 0 ,

$$
d_{N}=\frac{(N+1)(N+2)}{2} \text {. }
$$

Allowing for a spin-degeneracy of two, this predicts a shell structure for nuclei in which there are successive energy levels or shells that can be filled successively with up to $2,6,12,20,30,42,56, \ldots$ like nucleons, neutrons or protons. (The exclusion principle prohibits more than one identical nucleon in each state). By analogy with atomic physics, one would expect nuclei to be particularly stable when they have exactly the number of neutrons or protons or both to fill closed shells. Therefore the model predicts "magic numbers" of stability equal to 2, 8, 20, 40, 70, 112,... nucleons of either type. The first three of these agree rather well with experiment as the relative stability of $\mathrm{He}_{2}^{4}$ and $\mathrm{O}_{8}^{16}$ attests. However, to match the higher magic numbers that are observed in nature it is necessary to add what is called a spin-orbit coupling interaction to the central harmonic oscillator potential. This has the effect of depressing the energies of states with total angular momentum $j=l+\frac{1}{2}$ relative to those with $j=l-\frac{1}{2}$ with the result that some states get re-assigned to either higher or lower shells. The development of this model resulted in the award of a Nobel prize to Mayer and Jensen in 1966.

\section{The Hydrogenic Atom}

Another important application of Laguerre polynomials is in the solution of the Schrödinger equation for the hydrogenic atom. The equation is

$$
-\frac{\hbar^{2}}{2 m} \nabla^{2} \psi-\frac{Z e^{2}}{r} \psi=E \psi
$$


where $Z=1$ for hydrogen, $Z=2$ for singly ionized helium, and so on. Because the Coulomb potential is central, we have spherical symmetry again and can separate variables by setting

$$
\psi(\boldsymbol{r})=R(r) Y_{l}^{m}(\theta, \varphi)=\frac{u(r)}{r} Y_{l}^{m}(\theta, \varphi) .
$$

Once again, $l$ is a (conserved) orbital angular momentum. Substituting (11.5.45) into (11.5.44) we obtain the radial equation

$$
-\frac{\hbar^{2}}{2 m} \frac{1}{r^{2}} \frac{d}{d r}\left(r^{2} \frac{d R}{d r}\right)-\frac{Z e^{2}}{r} R(r)+\frac{\hbar^{2}}{2 m} \frac{l(l+1)}{r^{2}} R(r)=E R(r),
$$

or

$$
-\frac{\hbar^{2}}{2 m} \frac{d^{2} u}{d r^{2}}-\frac{Z e^{2}}{r} u(r)+\frac{\hbar^{2}}{2 m} \frac{l(l+1)}{r^{2}} u(r)=E u(r) .
$$

To render this into a recognizable form, we introduce some parameters similar to those used with the harmonic oscillator. We define

$$
\beta^{2}=-\frac{8 m E}{\hbar^{2}} \text { and } \lambda=\frac{2 m Z e^{2}}{\beta \hbar^{2}}
$$

where, recognizing that bound states correspond to negative values of $E$, we have put in a minus sign to ensure that $\beta$ is real. Then we introduce the new variables

$$
x=\beta r \text { and } y(x)=u(r)=u(x / \beta)
$$

in terms of which (11.5.47) becomes

$$
\frac{d^{2} y}{d x^{2}}-\left(\frac{l(l+1)}{x^{2}}-\frac{\lambda}{x}+\frac{1}{4}\right) y(x)=0 .
$$

The next step is to deduce the large and small $x$ behaviour of $y(x)$ by solving the asymptotic forms of the DE (11.5.49). For large $x$, we have

$$
\frac{d^{2} y}{d x^{2}}-\frac{1}{4} y(x) \simeq 0
$$

with solutions $y(x) \sim e^{ \pm \frac{x}{2}}$. Similarly, for small $x$ the DE becomes

$$
\frac{d^{2} y}{d x^{2}}-\frac{l(l+1)}{x^{2}} y(x) \simeq 0
$$

with solutions $x^{l+1}$ and $x^{-l}$. Since we require solutions that are bounded at both zero and infinity, we set

$$
y(x)=x^{l+1} e^{-\frac{x}{2}} v(x)
$$

and substitute into (11.5.49) to obtain the DE

$$
x \frac{d^{2} v}{d x^{2}}+(2 l+2-x) \frac{d v}{d x}+(\lambda-l-1) v(x)=0 .
$$


Once again this is the Laguerre $\mathrm{DE}$ (11.5.25) with polynomial solutions $L_{N}^{v}(x)$ provided that $2 l+1=v>-1$ and $\lambda-l-1=N$ is an integer or zero. The first condition is obviously satisfied and the second implies that $\lambda=N+l+1=n, n=1,2, \ldots$. This gives us the quantization rule

$$
\lambda=\frac{2 m Z e^{2}}{\beta \hbar^{2}}=\left(\frac{m}{-2 E}\right)^{\frac{1}{2}} \frac{Z e^{2}}{\hbar}=n,
$$

or

$$
E_{n}=-\frac{1}{2} m \frac{Z^{2} e^{4}}{\hbar^{2}} \frac{1}{n^{2}}, \quad n=1,2,3, \ldots
$$

or, expressed in terms of the "fine structure constant" $\alpha=\frac{e^{2}}{\hbar c}$,

$$
E_{n}=-\frac{1}{2} m c^{2}\left(Z \alpha^{2}\right) \frac{1}{n^{2}}, n=1,2,3, \ldots
$$

This agrees with the (Nobel prize winning) result that Neils Bohr obtained from an application of the somewhat heuristic Bohr-Sommerfeld quantization rules .

From (11.5.48), we have

$$
\beta \equiv \beta_{n}=2 \frac{m Z e^{2}}{\hbar^{2}} \frac{1}{n}=\frac{2 Z}{n a_{0}} \text { where } a_{0}=\frac{\hbar^{2}}{m e^{2}} \equiv \text { the Bohr radius. }
$$

Then, reconstructing the solution of the Schrödinger equation and using the Laguerre polynomial normalization given in (11.5.24), we find that the normalized hydrogenic wave function is

$$
\psi_{n l m}(\boldsymbol{r})=\left[\left(\frac{2 Z}{n a_{0}}\right)^{3} \frac{(n-l-1) !}{2 n(n+l) !}\right]^{\frac{1}{2}} e^{-\frac{\beta_{n} r}{2}}\left(\beta_{n} r\right)^{l} L_{n-l-1}^{2 l+1}\left(\beta_{n} r\right) Y_{l}^{m}(\theta, \varphi) .
$$

Since $E_{n}$ depends only on $n$, there is degeneracy with respect to both $m$ (due to the spherical symmetry of the problem) and $l$ (due to a higher, "accidental" symmetry of the wave equation). For each value of $n, l$ can vary from 0 to $n-1$ and for each of these $l$ values $m$ can vary from $-l$ to $l$. Thus, the degree of degeneracy is

$$
d_{n}=\sum_{l=0}^{n-1}(2 l+1)=2 \frac{n(n-1)}{2}+n=n^{2} .
$$

Allowing for spin degeneracy, this means that the $n t h$ level (shell) of the hydrogenic atom contains $2 n^{2}$ states. 